\title{
1 Neuronal circuitry underlying female aggression in Drosophila
}

3 Catherine E. Schretter ${ }^{1}$, Yoshinori Aso ${ }^{1}$, Marisa Dreher ${ }^{1}$, Alice A. Robie ${ }^{1}$, Michael-John Dolan ${ }^{1}$,

$4 \quad{ }^{2}$, Nan Chen ${ }^{1}$, Masayoshi Ito ${ }^{1}$, Tansy Yang ${ }^{1}$, Ruchi Parekh ${ }^{1}$, Gerald M. Rubin ${ }^{1}$

$6 \quad{ }^{1}$ Janelia Research Campus, Howard Hughes Medical Institute, Ashburn, VA 20147, USA

$7 \quad{ }^{2}$ Current address: Department of Neurology and F.M. Kirby Neurobiology Center, Boston

8 Children's Hospital, Harvard Medical School, Boston, Massachusetts 02115, USA

9

$10 *$ Correspondence: rubing@janelia.hhmi.org 


\section{Abstract}

13

14 Aggressive social interactions are used to compete for limited resources and are regulated by

15 complex sensory cues and the organism's internal state. While both sexes exhibit aggression, its

16 neuronal underpinnings are understudied in females. Here, we describe a set of connected

17 neurons in the adult Drosophila melanogaster central brain that drive female aggression. We

18 identified a population of sexually dimorphic aIPg neurons whose optogenetic activation

19 increased, and genetic inactivation reduced, female aggression. Analysis of GAL4 lines

20 identified in an unbiased screen for increased female chasing behavior revealed the involvement

21 of another sexually dimorphic neuron, $\mathrm{pC} 1 \mathrm{~d}$, and implicated $\mathrm{pC} 1 \mathrm{~d}$ and aIPg neurons as core

22 nodes regulating female aggression. pC1d activation increased female aggression and electron

23 microscopy (EM) connectomic analysis demonstrated that aIPg neurons and $\mathrm{pCld}$ have strong

24 reciprocal connections. Our work reveals important regulatory components of the neuronal

25 circuitry that underlies female aggressive social interactions and provides tools for their

26 manipulation. 


\section{Introduction}

Aggressive behaviors are important for gaining access to resources, including food and territory, and are exhibited by both sexes in multiple species (Anderson, 2016; Kravitz and Huber, 2003; Zwarts et al., 2012). As aggressive actions carry the risk of injury, strict regulation of aggression is needed to facilitate survival. Sensory information about the presence of other individuals and the nature of the surrounding environment strongly modulate aggressive social interactions (Chen and Hong, 2018; Hoopfer, 2016). However, understanding the neuronal mechanisms by which such stimuli influence aggression has been hindered by a lack of knowledge about the structure of the underlying neuronal circuits, particularly in females.

Centers mediating, or conveying the information necessary for, aggression have been identified in the medial hypothalamus through classic experiments using electrical stimulation in cats and rodents (Albert et al., 1979; Bandler et al., 1972; Berntson, 1973; Chi and Flynn, 1971; Gregg, 2003; Kruk et al., 1983; Lammers et al., 1988; Siegel et al., 1999; Takahashi and Miczek, 2015; Woodworth, 1971). Such key regions are thought to perform a different role than other brain areas that facilitate aggressive interactions by altering the overall level of social behavior (Siegel et al., 1999). Recent work using opto- and chemo-genetic techniques have narrowed down these key regions to small populations of cells in mice, including those expressing estrogen receptor alpha (Esr1) and progesterone receptor (PR) in the ventrolateral part of the ventromedial hypothalamus (VMHvl) (Hashikawa et al., 2017; Lee et al., 2014; Yang et al., 2013). While $\mathrm{Esr}^{+}$neurons in the VMHvl regulate aggression in both male and female mice, there are sex differences in the populations involved (Hashikawa et al., 2017). Additionally, the VMHvl has also been implicated in other female sexual behaviors (Hashikawa et al., 2017; Lee et al., 2014; 
52 Pfaff and Sakuma, 1979a, 1979b; Yang et al., 2013), further complicating the identification of

53 the specific cells that mediate aggressive interactions.

54

55

56

57

58

Since the first observation of aggressive behaviors in Drosophila by Sturtevant in 1915, social behaviors associated with attack and threat displays in flies have been well described ethologically (Shelly, 1999; Sturtevant, 1915; Ueda and Kidokoro, 2002; Zwarts et al., 2012). While male aggression is heightened in the presence of mate-related cues, female flies display increased aggressive encounters in the presence of limited nutrients, such as yeast, and when near egg laying sites (Bath et al., 2017, 2018; Lim et al., 2014; Shelly, 1999; Ueda and Kidokoro, 2002). Additionally, social isolation can increase aggression in both male and female flies (Hoffmann, 1990; Ueda and Kidokoro, 2002). As in mammals (Hashikawa et al., 2017), aggressive behaviors in flies include sex-specific components, such as head butting in female flies, as well as those that are shared between the sexes (Nilsen et al., 2004). Due to the complexity of the behavior and the sensory stimuli that influence its presentation, a circuit diagram would greatly facilitate understanding the underlying neuronal mechanisms. To gain a mechanistic understanding of how these behaviors are regulated and executed, we will need to identify the specific cells that contribute in each sex and place them in the context of larger neuronal circuits.

\section{The fruit fly, Drosophila melanogaster, provides a good model for dissecting the neuronal} circuitry of aggression due to the genetic tools available for targeting and manipulating individual cell types, the availability of extensive connectomic information, and the relative simplicity of its nervous system and behavior (Bellen et al., 2010; Dionne et al., 2018; Kravitz 
and Huber, 2003; Scheffer et al., 2020; Simpson and Looger, 2018; Tirian and Dickson, 2017).

In male flies, studies investigating the neuronal correlates of aggression have implicated a group of 18 - 34 cells in the central brain, the $\mathrm{P} 1 / \mathrm{pC} 1$ cluster, as well as various neuropeptides and biogenic amines, including neuropeptide F, tachykinin, and octopamine (Alekseyenko et al., 2019, 2014; Asahina, 2018, 2017; Asahina et al., 2014; Dierick and Greenspan, 2007; Hoopfer et al., 2015; Hoyer et al., 2008; Ishii et al., 2020; Wohl et al., 2020; Wu et al., 2020; Zhou et al., 2008). However, research on female aggressive social interactions has been less extensive as

83 females exhibit less aggression under the behavioral conditions used for males. There are also sex differences in the behavioral components and underlying neurons important for aggression (Hoopfer et al., 2015; Nilsen et al., 2004). Genes involved in sexual differentiation, including doublesex (dsx) and fruitless (fru), contribute to sexual behaviors (Dickson, 2008; Pavlou and Goodwin, 2013; Siwicki and Kravitz, 2009; Yamamoto, 2007; Yamamoto and Koganezawa, 2013; Zhou et al., 2014). Recent work has revealed the involvement of the doublesex-expressing $\left(d s x^{+}\right) \mathrm{pC} 1$ cluster, a group of 5 cell types, in promoting aggressive phenotypes in female flies

(Fathy, 2016; Palavicino-Maggio et al., 2019). As in the VMHvl of mice, cells within this cluster can be divided into multiple subtypes and particular subtypes have been shown to also be involved in other female behaviors, including mating and egg laying (Wang et al., 2020).

93 Understanding the flow of the information within the neuronal circuit will require knowledge of

94 which cells within the $\mathrm{pC} 1$ cluster contribute preferentially or exclusively to aggressive

95 behaviors.

96

97 Recent advances in connectomics, combined with previously established genetic tools for

98 manipulating neuronal cell types, facilitate systematic and comprehensive studies of the circuitry 
100 morphologically similar, sexually dimorphic, cholinergic, and fruitless-positive neurons, a subset

101 of the aIP-g neurons described by Cachero et al. (2010). We found that optogenetic activation of

102 these cells, even in the absence of aggression-promoting environmental conditions, increased

103 female aggression. This behavior extended beyond the stimulation period, similar to the

104 persistent phenotype generated by P1 neuronal activation in males (Hoopfer et al., 2015).

105 Importantly, blocking aIPg synaptic transmission resulted in diminished female aggression,

106 suggesting they modulate wild-type interactions. We then identified a second cell type, a

107 particular $\mathrm{pC} 1$-subtype ( $\mathrm{pC} 1 \mathrm{~d})$, that also induces aggression upon activation. Analysis of a

108 connectome of a large part of the fly central brain (Scheffer et al., 2020) revealed that aIPg and

$109 \mathrm{pCld}$ neurons are reciprocally and strongly connected, consistent with a coordinated role in

110 female aggression. Taken together, our work yields insights into female aggressive behavior and

111 identifies the two cell types that appear to form the key nodes of the circuit that implements these

112 behaviors.

113

114

115

116

117

118

119

121

122

123

124

125

\section{Results}

\section{Identification of neurons modulating female aggressive behaviors}

In a behavioral screen using split-GAL4 lines to examine another phenotype, we noted a

dramatic increase in head butting and fencing behaviors, known components of female aggression (Bath et al., 2017; Nilsen et al., 2004; Palavicino-Maggio et al., 2019; Ueda and

Kidokoro, 2002), upon stimulation of one neuronal subset of approximately eleven cells (see

Video 1 compared with Video 2). We generated multiple, independent split-GAL4 lines (Dionne

$$
\text { et al., 2018; Luan et al., 2006; Pfeiffer et al., 2010) labelling this same neuronal population. }
$$


126 Group-housed virgin females from these lines expressing the red-shifted opsin CsChrimson were

127 then screened for behavioral changes upon light activation (Kim et al., 2015; Klapoetke et al.,

128 2014). We tracked freely moving flies within a standardized $127 \mathrm{~mm}$ arena using video-assisted

129 tracking software to perform automated behavioral analyses (Branson et al., 2009; Robie et al.,

130 2017; Simon and Dickinson, 2010). These lines all exhibited similar increases in aggressive

131 behaviors, such as fencing and head butting (Figure 1, Figure 1-figure supplements 1, 2 and 3;

132 compare Videos 1 and 2). Consistent with the behaviors being optogenetically induced, these

133 interactions were virtually absent when all trans-retinal was omitted from the food (Figure 1-

134 figure supplement 4).

136 To characterize these cells, we used whole mount immunohistochemistry and confocal imaging

137 with an mVenus-tagged CsChrimson reporter. These lines labelled a set of neurons with cell

138 bodies in the inferior protocerebrum and major projections in the anterior optic tubercle (AOTU),

139 anterior ventrolateral protocerebrum (AVLP), superior medial protocerebrum (SMP), and

140 superior intermediate protocerebrum (SIP) (Figure 1A, Figure 1-figure supplements 1 and 2).

141 The cells observed in our split-GAL4 lines morphologically resemble, and appear to be a subset

142 of, the 32 aIP-g neurons described in Cachero et al. (2010), which were previously classified as

$143 \mathrm{fru}^{+}$auditory interneurons with sexually dimorphic projections. We refer to this subset as aIPg.

144 In two of our aIPg lines (aIPgSS1 and aIPgSS4), no expression was seen in males (Figure 1-

145 figure supplement 1C). We tested for changes in male behavior in the aIPgSS1 line and found no

146 differences (Figure 1-figure supplement 5), which was unsurprising given the absence of

147 detectable expression of this line in males (Figure 1 - figure supplement 1). Transcript analysis 148 of aIPg split-GAL4 lines confirmed the expression of fruitless $($ fru $)$ as well as genes associated 
149 with use of acetylcholine as a neurotransmitter, which is consistent with previous descriptions

150 (Cachero et al. 2010), as well as and short neuropeptide F (sNPF) (Figure 1-figure supplement

$1516)$.
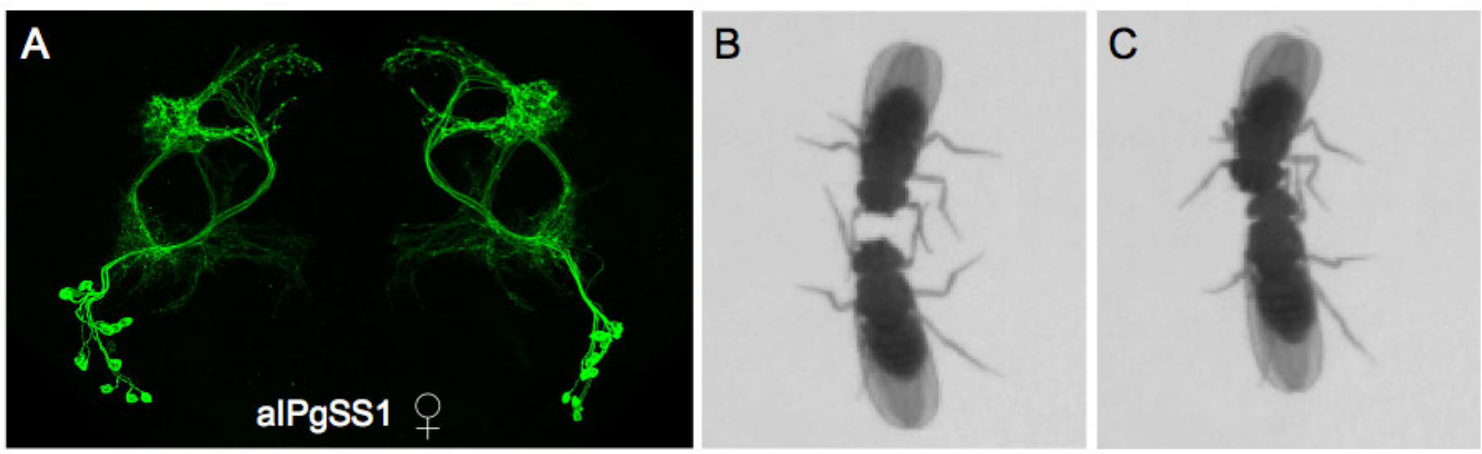

D

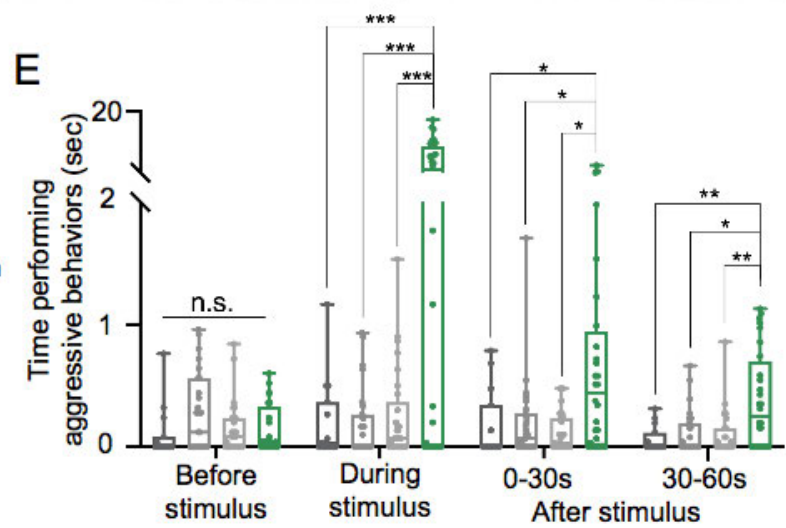

Figure 1. Optogenetic activation of 11 aIPg neurons results in an increase in female aggressive behaviors. (A) Maximum intensity projection (63x) image of the central brain of a female from the aIPgSS1 line crossed with 20xUAS-CsChrimson::mVenus and stained with anti-GFP antibody. Images of the complete brain and ventral nerve cord of a female and male of the same genotype are shown in Figure 1- figure supplement 1A - C. Images of individual aIPg neurons, generated by stochastic labeling are shown in Figure 1- figure supplement 2. (B, C) Images of female flies displaying fencing (B) and head butting (C) behaviors. (D) Percentage of flies engaging in aggressive behaviors, defined as displaying fencing or head butting, as measured by a JAABA classifier over the course of a 2minute trial during which a 30 -second $0.4 \mathrm{~mW} / \mathrm{mm}^{2}$ continuous light stimulus was delivered. The red shading indicates the stimulus period. The mean is represented as a solid line and shaded error bars represent standard variation between experiments. Each experiment included approximately 15 flies. See Figure 1 - figure supplement $7 \mathrm{G}$ for additional quantification. For a description of the JAABA classifier, see Materials and Methods. 20xUASCsChrimson, $\mathrm{n}=3$ experiments; aIPgSS1, $\mathrm{n}=3$ experiments; EmptySS $>20 \mathrm{xUAS}$-CsChrimson, $\mathrm{n}=3$ experiments; aIPgSS1 $>20 x U A S-C s C h r i m s o n, ~ n=3$ experiments. (E) Total time an individual spent performing aggressive behaviors during each of four 30-second periods: prior to, during, immediately following, and 30-60 sec after the stimulus. Points represent individual flies: 20xUAS-CsChrimson, $\mathrm{n}=15$ flies; aIPgSS1, $\mathrm{n}=27$ flies; EmptySS $>20 x U A S-C s C h r i m s o n, ~ n=29$ flies; aIPgSS1 > 20xUAS-CsChrimson, $n=28$ flies. Box-and-whisker plots show median and interquartile range (IQR); whiskers show range. A two-way ANOVA with a Tukey's multiple comparisons post-hoc test was used for statistical analysis. Asterisk indicates significance from 0 : ${ }^{*} \mathrm{p}<0.05$; $* * \mathrm{p}<0.01 ; * * * \mathrm{p}<0.001 ; \mathrm{n} . \mathrm{s}$, not significant. 
154 Aggressive behaviors have multiple components. We began by quantifying three such behaviors,

155 chasing, touching, and walking, using a set of previously created and validated automatic

156 behavior classifiers (Robie et al., 2017). We found that flies increased touching compared to the

157 empty split-GAL4 control during a 30-second stimulation (Figure 1-figure supplement 7A - B).

158 A low level of chasing was also detected upon activation (Figure 1-figure supplement 7C - D).

159 Although the average walking velocity of the flies over the course of the trial did not differ

160 compared to controls, a sharp decrease in the percent of flies walking followed stimulus onset

161 (Figure 1-figure supplement 7E - F). Examination of behavior metrics for individual flies, or

162 per-frame features, revealed a significant increase in the number of flies within two body lengths

163 during stimulation; moreover, apparent orientation of flies towards one another can be easily

164 observed, suggestive of visually directed movement (Figure 1-figure supplement 7H; compare

165 Videos 1 and 2). Additionally, behavioral changes were both diminished and delayed if

166 stimulation experiments were conducted in the absence of visible light (Figure 1-figure

167 supplement 8), indicating that vision is important in the initial phase of these actions.

169 Touching and chasing are components of social behaviors, including courtship and aggression, in

170 male flies (McKellar et al., 2019; Nilsen et al., 2004). While certain features of aggression are

171 shared between males and females, there are sex-specific aspects, including head butting and the

172 way in which behavioral patterns progress during an encounter (Nilsen et al., 2004). To examine

173 female-specific attributes, we generated and validated a new JAABA classifier for female

174 aggression (Supplementary Table 1). An aggressive event was defined as either an instance of

175 fencing (Figure 1B) and/or head butting (Figure 1C), as these behaviors were not always

176 distinguishable at the image resolution used for quantification. Examples of fencing and head 
177 butting are shown at a high resolution and frame rate in Video 3. Female aggression

178 encompasses a range of behaviors involved in attack and threat displays; however, in this paper

179 we used the term "aggression" in a limited way to refer to fencing and head butting behaviors.

180 Employing this classifier, we found that a 30-second stimulation of lines labelling aIPg neurons

181 increased the number of flies engaged in aggressive behaviors as well as the amount of time

182 individuals spent in such activities (Figure 1D - E, Figure 1-figure supplement 7G, Videos 1 and

183 2). These behaviors extended beyond the activation period (Figure $1 \mathrm{D}-\mathrm{E}$ ), suggesting a

184 persistent internal state.

186 Both the expression level of the effector and the light intensity used for optogenetic stimulation

187 can influence behavior and, in extreme cases, be cytotoxic (unpublished observations; Kim et al.,

188 2015). Higher levels of stimulation also increase the possibility that cells expressing the effector

189 at levels too low for detection with confocal microscopy may contribute to the observed

190 behavior. For these reasons, we examined the expression patterns of our split-GAL4 lines with

191 the highest level of the effector used in our behavioral experiments and did not detect expression

192 in other cell types or obvious toxicity in the aIPg cells (Figure 1, Figure 1-figure supplement 1).

193 We also conducted experiments using 5xUAS-, 10xUAS-, and 20xUAS-CsChrimson constructs

194 that are expected to produce a four-fold range of effector expression (Pfeiffer et al 2010; Figure

195 1-figure supplement 9A, C). Finally, we varied light intensity over a 10-fold range, 0.04, 0.1 and

$1960.4 \mathrm{~mW} / \mathrm{mm}^{2}$ (Figure 1-figure supplement 9B). Similar effects on aggressive behavior were

197 found under all conditions, strongly supporting the conclusion that the cell types we observe in

198 the split-GAL4 lines are mediating the observed phenotypes. 
200 We examined the effect of the stimulus frequency, as this can affect changes in the behavioral

201 state (Hoopfer et al., 2015). We modified the stimulus delivery from the constant protocol used

202 previously to a pulse stimuli. Delivery of a $5 \mathrm{~Hz} 0.1 \mathrm{~mW} / \mathrm{mm}^{2}$ stimulus $(5 \%$ duty cycle, or

203 fraction of time during which the stimulus is on) over 30 seconds induced significant behavioral

204 changes (Figure 1-figure supplement 10A - C). Changing the frequency and duty cycle $(10 \mathrm{~Hz}$,

$2050.1 \mathrm{~mW} / \mathrm{mm}^{2}$ stimulus, $10 \%$ duty cycle) increased aggressive behaviors, but frequencies beyond

$20610 \mathrm{~Hz}$ did not significantly change behavior from the levels observed at $10 \mathrm{~Hz}$. However, higher

207 frequencies did increase aggression during the off period, suggesting that increasing the

208 frequency and/or total intensity of the stimulation can increase persistence (Figure 1-figure

209 supplement 10A - D). These experiments demonstrate that aIPg neurons promote female

210 aggressive interactions under a range of stimulus conditions, and, at least under certain stimulus

211 protocols, can cause a change in brain state that continues beyond the stimulus period.

aIPg neurons mediate wild type female aggressive social interactions

The infrequent occurrence of female aggressive events (Bath et al., 2017; Shelly, 1999; Ueda and

216 Kidokoro, 2002), at least under laboratory conditions, has made it difficult to study its neuronal

217 correlates. To facilitate such experiments, we optimized environmental conditions to increase the

218 level of female aggression in wild-type files. Alterations to diet and life history are known to

219 increase female aggression in wild-type flies (Bath et al., 2017; Ueda and Kidokoro, 2002). We

220 varied these parameters and arena size to observe interactions between pairs of flies. A $1 \mathrm{~mm}$

221 spot of yeast was also included within the arena and the diet of the flies was adjusted to restrict

222 protein for 20 to 24 hours prior to testing (Ueda and Kidokoro, 2002). Under these conditions,

223 we observed sufficient levels of aggression to examine the effects of inactivation of aIPg neurons 
224 with the synaptic inhibitor tetanus toxin (Sweeney et al., 1995). A significant reduction in the

225 time spent performing aggressive behaviors, as measured using both manual and automated

226 behavioral analyses, was observed with three different split-GAL4 lines (Figure 2, Figure 2-

227 figure supplement $1 \mathrm{~A}-\mathrm{B}$, and Figure 2-figure supplement 2A - D). Such changes did not appear

228 to be due to decreased movement as flies exhibited similar or higher velocity compared to

229 controls over the 30-minute trial (Figure 2-figure supplement 1C-D). These results indicate that

230 aIPg neurons are important for modulating aggressive behaviors in wild-type females.

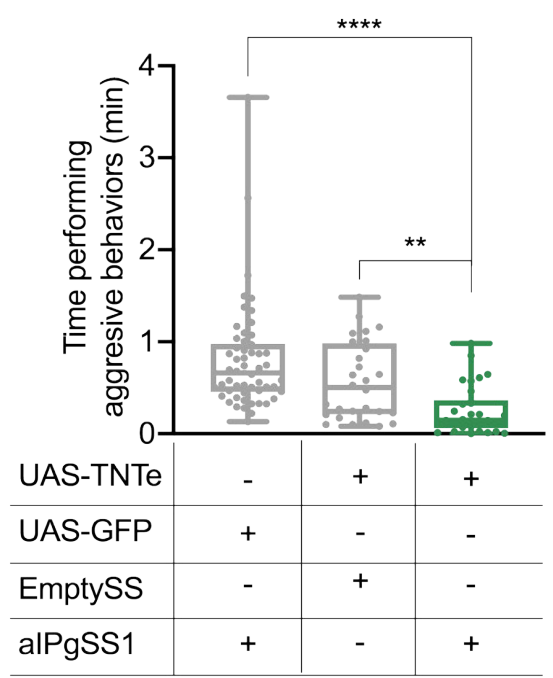

Figure 2. aIPg inactivation results in diminished aggressive social interactions.

Total time an individual spent performing aggressive behaviors over a 30 minute trial. Individuals were pooled over 2 independent testing days during the same week. Points indicate individual flies. aIPgSS1 > UASGFP, $\mathrm{n}=54$ flies; EmptySS > UAS-TNTe, $\mathrm{n}=28$ flies; aIPgSS1 > UASTNTe, $\mathrm{n}=30$ flies. Box-and-whisker plots show median and interquartile range (IQR); whiskers show either $1.5 \times \mathrm{IQR}$ of the lower and upper quartiles. Kruskal-Wallis and Dunn's post hoc tests were used for statistical analysis. Asterisk indicates significance from 0 : $* * \mathrm{p}<0.01$; $* * * * \mathrm{p}<0.0001$.

\section{Activation of aIPg overrides the requirement for specific environmental conditions for}

\section{6 female aggressive behaviors}

247 In addition to food availability, the genotype and sex of the target fly influence aggression (Bath

248 et al., 2020, 2018; Lim et al., 2014; Ueda and Kidokoro, 2002). Our previous neuronal activation

249 experiments demonstrated aggression even in the absence of competition for food (Figure 1D -

250 E). We investigated the effects of activation status and the sex of the opponent in experiments

251 using pairs of flies. Irrespective of whether aIPg neurons were stimulated in the both females,

252 photostimulation in the absence of food increased the total time spent displaying aggression and

253 decreased attack latency, or the time from stimulus on until the first aggressive event (Figure 3A 
- B, Figure 3-figure supplement 1 A - B, and Video 3). Females in which aIPg neurons were

figure supplement $1 \mathrm{C}$, and Video 4). Mating behaviors share neuronal circuitry with aggression

associated with increased aggression in wild-type females.

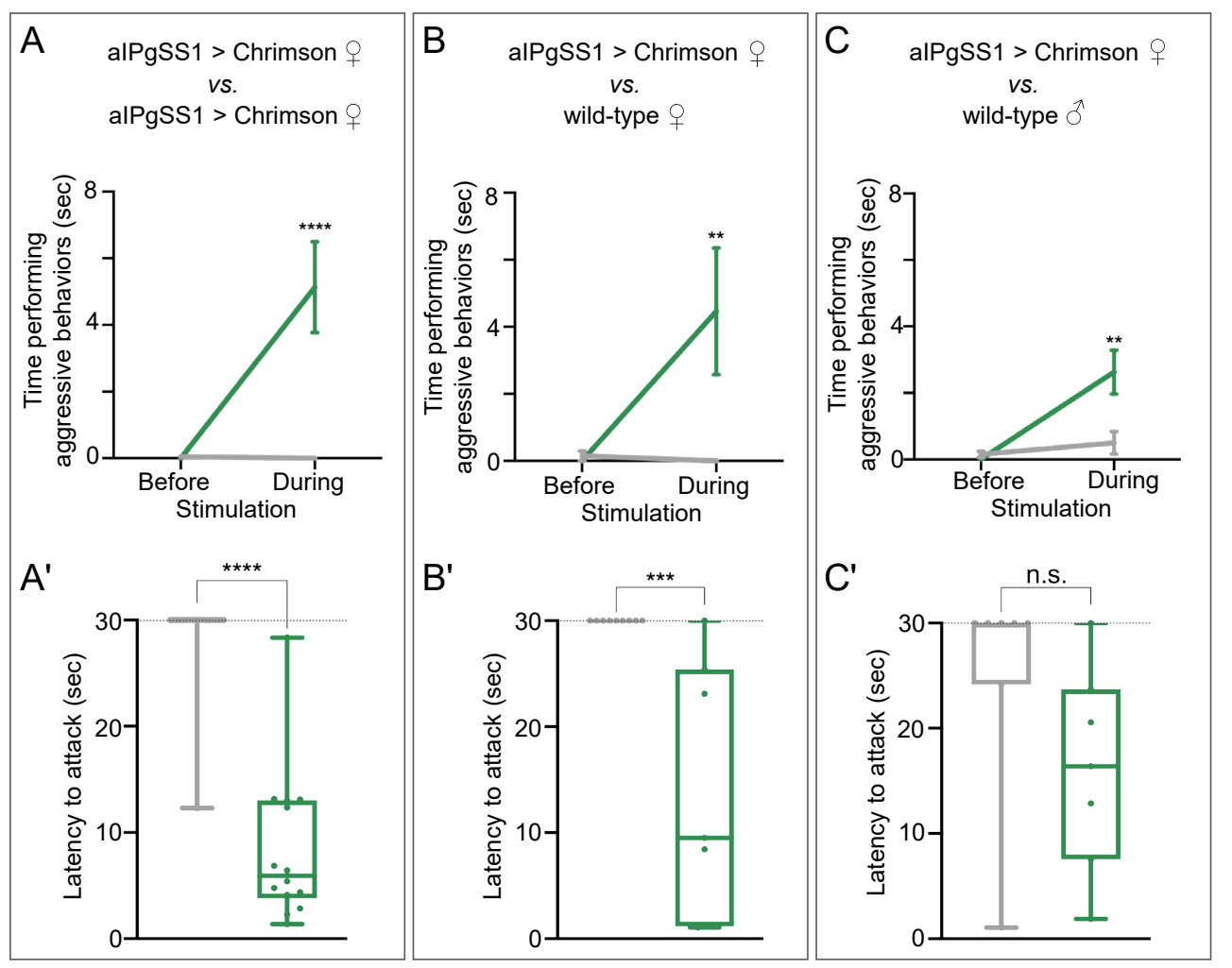

Figure 3. aIPg activation increases aggression against wild-type females and males.

(A-C) Total time an individual spent performing aggressive behaviors in a $16 \mathrm{~mm}$ arena over the 30 second period prior to or during stimulation. The plots refer only to the behavior of aIPgSS1 > Chrimson females and each arena contained only two flies: (A) Two aIPgSS1 > Chrimson females; (B) an aIPgSS1 > Chrimson female and a wild-type (Canton-S) female; and (C) a aIPgSS1 > Chrimson female and a wild-type (Canton-S)

male. The green line shows the stated genotype; the gray line shows the results when EmptySS $>$ Chrimson was used instead of aIPgSS1 > Chrimson. $\left(\mathrm{A}^{\prime}-\mathrm{C}^{\prime}\right)$ Amount of time during a 30 second $0.1 \mathrm{~mW} / \mathrm{mm}^{2}$ continuous stimulation period until first aggressive encounter. Points indicate individual flies. Dotted lines indicate the end of the trial and error bars are mean +/- S.E.M. Box-and-whisker plots show median and interquartile range (IQR); whiskers show either $1.5 \times$ IQR of the lower and upper quartiles. A': EmptySS $>20 x U A S-C h r i m s o n, ~ n=22$ flies; aIPgSS1 > 20xUAS-Chrimson, $\mathrm{n}=14$ flies; B': EmptySS > 20xUAS-Chrimson, $\mathrm{n}=8$ flies; aIPgSS1 $>$ 20xUASChrimson, $\mathrm{n}=7$ flies; $\mathrm{C}^{\prime}$ : EmptySS $>$ 20xUAS-Chrimson, $\mathrm{n}=7$ flies; aIPgSS $1>20 x$ UAS-Chrimson, $\mathrm{n}=7$ flies. A Mann-Whitney $U\left(\mathrm{~A}^{\prime}-\mathrm{C}^{\prime}\right)$ post hoc test or a two-way ANOVA with a Sidak's multiple comparisons post-hoc test $(A-C)$ was used for statistical analysis. Asterisk indicates significance from $0:{ }^{*} p<0.05 ;{ }^{* *} \mathrm{p}<0.01 ;{ }^{* * * *} \mathrm{p}<0.0001$; n.s., not significant. 


\section{Identifying additional cell types involved in mediating female aggression}

264 Having established a role for aIPg neurons in female aggression, we used two complementary

265 methods to discover additional cells involved in regulating this behavior. First, we used

266 behavioral screens to identify other cell types that could drive female aggression when activated.

267 Second, we used the aIPg neurons as an entry point for EM-based circuit mapping. As described 268 below, both approaches converged on the same set of cells.

aIPg and pC1d are two key groups of neurons involved in female aggressive behaviors

271 It is reasonable to expect that other neurons in the circuit or parallel pathways could also induce aggression upon activation. To identify such neurons, we took a strategy analogous to that used

In this way, the number of different genes that can give rise to the phenotype under study when

278 further cell types that could also increase female aggression, we analyzed the results of a

279 previous screen of over 2,000 GAL4 lines, in which cell types are expressed in multiple lines

280 (Robie et al., 2017). We generated split-GAL4 hemi-driver lines using the enhancers from this

281 screen's top hits for increased female-female chasing behavior and crossed them to each other to

282 reveal the presence of shared cell types (Figure 4; Figure 4-figure supplement 1). Strikingly, 13

283 of the top 14 hits based upon their behavioral score could be accounted for by just two cell types:

284 aIPg and $\mathrm{pC} 1 \mathrm{~d}$, one of the five cell types found in the $\mathrm{pC} 1$ cluster of cells in females. Members 
A

\begin{tabular}{|c|c|c|c|}
\hline LINE & Z-score & Cell type & $\begin{array}{c}\text { Panel in } \\
\text { Fig. suppl. }\end{array}$ \\
\hline R26F09 & 352 & pC1d & A \\
\hline R26E01 & 324 & pC1d & B \\
\hline R44D11 & 138 & pC1d & C \\
\hline R21A01 & 123 & pC1d & D \\
\hline R72C11 & 104 & alPg & E \\
\hline R20C08 & 79 & pC1d & F \\
\hline R45F11 & 59 & pC1d & G \\
\hline R51B06 & 55 & pC1d & H \\
\hline R71A09 & 48 & pC1d & A, C F \\
\hline R30G01 & 45 & pC1d & D, I \\
\hline R65G11 & 44 & pC1d & D \\
\hline R48F12 & 37 & N.D. & - \\
\hline R23A07 & 37 & alPg & E \\
\hline R24D02 & 36 & pC1d & G \\
\hline
\end{tabular}
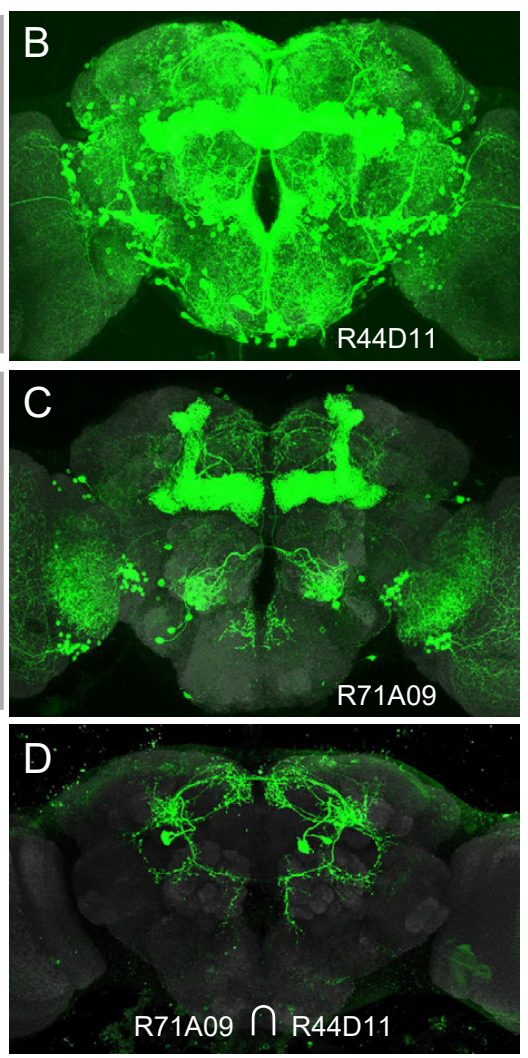

Figure 4. An unbiased screen suggests that aIPg1 - 3 and pC1d are key nodes for gating female-female aggression.

(A) The fourteen top hits for female-female chasing from an unbiased activation screen of 2,204 generation 1 GAL4 lines are listed along with their Z-scores for this behavior as determined by Robie et al. (2017). Also shown is the relevant cell type we concluded from our intersectional analysis (Figure 4-figure supplement 1) to be present in each line. N.D., no cell type reproducibly detected, suggesting that R48F12 may not share a common cell type with any of the other 13 lines. The final column in the table refers to the panels in Figure 4-figure supplement 1 where results supporting the stated conclusion are shown. (B, C) The expression patterns of the two indicated GAL4 lines (Jenett et al. 2012). The images shown were taken from the database at www.janelia.org/gal4-gen1, where the expression patterns of the other lines listed in A can also be found. (D) The expression pattern of a split-GAL4 line made by intersecting these two enhancers; a cell with the morphology of pC1d can be seen.

290 The five cell types that make up the pC1 group express $d s x$ and have been collectively implicated

291 in female receptivity, oviposition, male courtship, and both male and female aggression (Fathy,

292 2016; Hoopfer et al., 2015; Ishii et al., 2020; Palavicino-Maggio et al., 2019; Rideout et al., 
2010; Scheffer et al., 2020; Wang et al., 2020; Wohl et al., 2020; Zhou et al., 2014). In previous work, it has proven difficult to elucidate the relative contribution of the five different $\mathrm{pC} 1 \mathrm{cell}$ types present in females to these behaviors. pC1d cells are revealed in intersections using the enhancers from 11 of the top 14 hits from the Robie et al. (2017) screen (Figure 4; Figure 4-

297 figure supplement 1). However, nearly all of these intersections contain at least one other $\mathrm{pC} 1$

298 cell type, leaving open the possibility that inducing aggression requires a combination of 299 multiple $\mathrm{pC} 1$ cell types.

\section{Activation of pC1d alone, but not pC1e or pC1a - c, promotes female aggressive behaviors}

To address the role of individual pC1 cell types in female aggression, we generated split-GAL4

304 lines that drive expression in either $\mathrm{pC} 1 \mathrm{~d}$ or $\mathrm{pC} 1 \mathrm{e}$ as well as lines containing both cell types

305 (Figure 5A, Figure 6A, Figure 5-figure supplements 1 and 2, and Figure 6-figure supplement 1

and 2). We also used a split-GAL4 line that labels pC1a - c (provided by K. Wang and B.

307 Dickson). No expression was observed in males in the majority of the lines used (Figure 5-figure

308 supplement 1C and Figure 6-figure supplement 1C). The cells labeled in males in two of the

309 lines were not morphologically similar to $\mathrm{pC} 1 \mathrm{~d}$ or $\mathrm{pC} 1 \mathrm{e}$ and these lines were not used for

310 behavioral analysis in males. Transcriptional profiling of $\mathrm{pC} 1 \mathrm{~d}$ and $\mathrm{pC} 1 \mathrm{e}$ confirmed that the cells

311 in females expressed $d s x$ and genes associated with use of acetylcholine (Figure 1-figure

312 supplement 5), as previously described (Palavicino-Maggio et al., 2019; Rezával et al., 2016;

313 Rideout et al., 2010; Zhou et al., 2014). Our data suggest that these cells might also express fru

314 (Figure 1-figure supplement 5). 


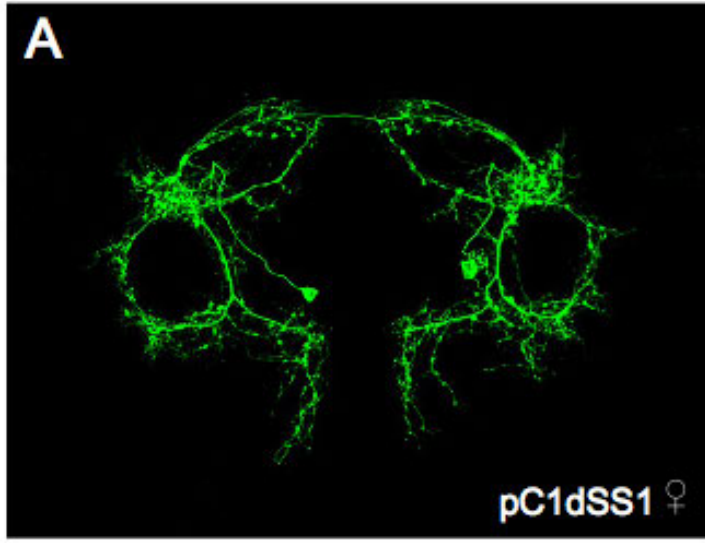

C
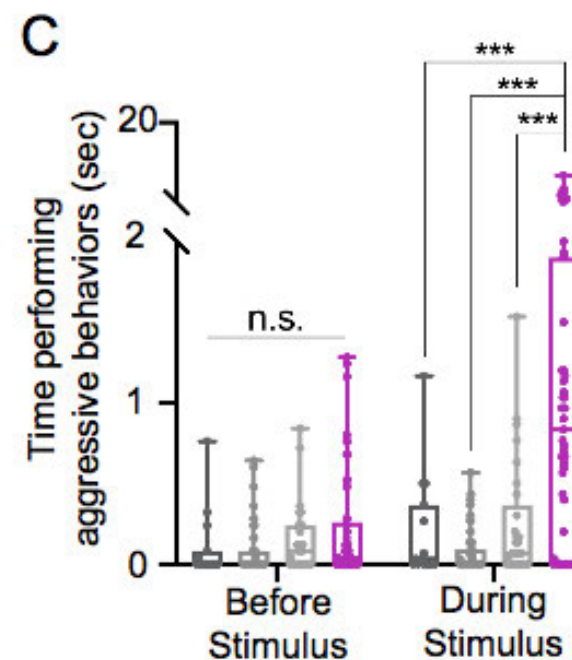

B

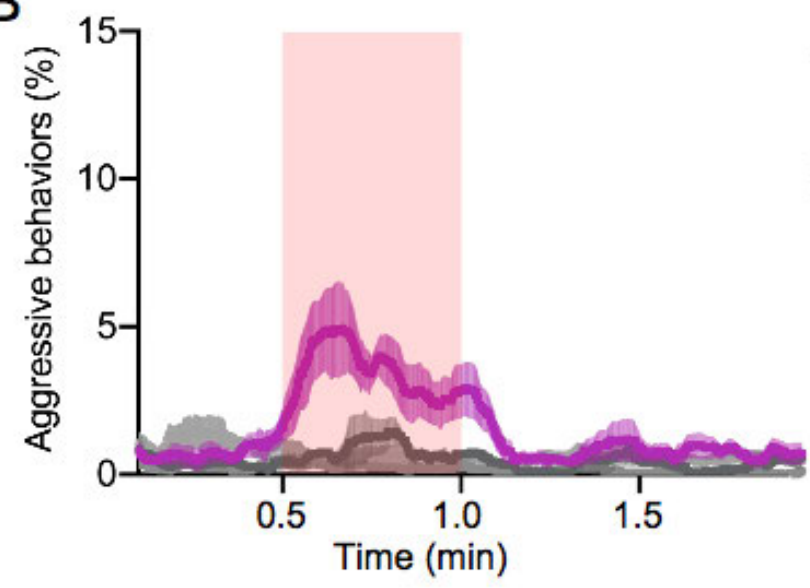

- UAS-Chrimson

n.s.

n.s.

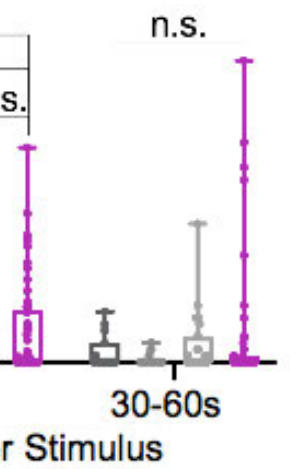

- pC1dSS1

- EmptySS > Chrimson

- pC1dSS1 > Chrimson

Figure 5. pC1d significantly increases aggressive social interactions in female flies.

(A) Maximum intensity projection (63x) image of the central brain of a female from the pC1dSS1 split-GAL4 line crossed with 20xUAS-CsChrimson::mVenus and stained with anti-GFP antibody. Images of the complete brain and ventral nerve cord of a female and male of the same genotype are shown in Figure 5 - figure supplement 1A - C. (B) Percentage of flies engaging in aggressive behaviors over the course of a 2-minute trial during which a 30-second $0.4 \mathrm{~mW} / \mathrm{mm}^{2}$ continuous light stimulus was delivered. Mean is represented as a solid line and shaded error bars represent variation between experiments. Each experiment included approximately 15 flies. 20xUAS-CsChrimson, $\mathrm{n}$ $=3$ experiments; $\mathrm{pC} 1 \mathrm{dSS} 1, \mathrm{n}=3$ experiments; EmptySS > 20xUAS-CsChrimson, $\mathrm{n}=5$ experiments; $\mathrm{pC} 1 \mathrm{dSS} 1>$ 20xUAS-CsChrimson, $\mathrm{n}=5$ experiments. (C) Total time an individual spent performing aggressive behaviors during each of four 30-second periods: prior to, during, immediately following, and 30-60 sec after the stimulus. Points represent individual flies. 20xUAS-CsChrimson, $\mathrm{n}=15$ flies; $\mathrm{pC} 1 \mathrm{dSS} 1, \mathrm{n}=48$ flies; EmptySS $>$ 20xUASCsChrimson, $\mathrm{n}=29$ flies; $\mathrm{pC} 1 \mathrm{dSS} 1>20 x \mathrm{x} A S-C s C h r i m s o n, \mathrm{n}=53$ flies. Box-and-whisker plots show median and interquartile range (IQR); whiskers show range. A two-way ANOVA with a Tukey's multiple comparisons post-hoc test was used for statistical analysis. Asterisk indicates significance from 0 : ${ }^{*} \mathrm{p}<0.05 ; * * \mathrm{p}<0.001 ;$ n.s., not significant.

316 Using the same stimulation parameters as we used for lines labeling aIPg neurons, optogenetic

317 activation of $\mathrm{pC} 1 \mathrm{~d}$ alone increased the percentage of aggressive flies (Figure 5B, Figure 5-figure 
319 included fencing and head butting as observed upon aIPg activation (Video 6). pC1d stimulation

320 increased touching and chasing; however, there were no sharp decreases in walking (Figure 5-

321 figure supplement 3B - D). Similar results were seen with additional split-GAL4 lines labelling

322 pC1d (Figure 5-figure supplement 4), while behavioral changes were not observed upon

323 stimulation of males (Figure 5-figure supplement 5A-B). Additional controls in which all

324 trans-retinal was omitted from the food did not show an elevation in aggression following light

325 onset (Figure 5-figure supplement $6 \mathrm{~A}-\mathrm{B}$ ). Increasing the intensity and frequency of stimulation

326 did heighten behavior (Figure 5-figure supplement 7A, Figure 5-figure supplement 8A - C).

327 However, changes in the expression level of CsChrimson were, unexpectedly, inversely

328 correlated with behavior (Figure 5-figure supplement $7 \mathrm{~B}-\mathrm{C}$ ), suggesting that higher effector

329 expression levels might be detrimental to cell function.

331 Notably, we did not find significant continued changes in behavior following the stimulation

332 period for any of the frequencies tested using $\mathrm{pC} 1 \mathrm{~d}$, in contrast to the persistence of aggression

333 observed after similar activation of aIPg neurons (Figure 1-figure supplement 10A, B, D and

334 Figure 5-figure supplement 8A, B, D). As the behaviors induced by activation of aIPg neurons

335 were largely independent of external conditions, we tested whether pC1d behavioral effects were

336 similarly resistant to changes in the target or arena. pC1d stimulation resulted in similar attack

337 latency and time spent performing aggressive behaviors irrespective of whether the opponent

338 was a wild-type female or male (Figure 5-figure supplement 9A-C). While activation promoted

339 aggression, no differences were observed following pC1d inactivation with tetanus toxin (Figure

340 5-figure supplement 10). These results suggest that $\mathrm{pC} 1 \mathrm{~d}$ neurons are not essential for female 
341 aggression; however, we have not independently confirmed the degree of effectiveness of the

342 tetanus toxin inactivation.

344 In contrast to $\mathrm{pC} 1 \mathrm{~d}$, stimulation of lines containing $\mathrm{pC} 1 \mathrm{e}$ alone did not significantly alter any of

345 the behaviors we assayed (Figure 6B, Figure 6-figure supplement 1A - E, Figure 6-figure

346 supplement 2A - D, Figure 6-figure supplement 3A-B). Changes in the effector expression

347 levels, light power density, and frequency did not significantly alter behavior upon activation of

$348 \mathrm{pCle}$ (Figure 6-figure supplement 4A - D), suggesting that the absence of behavioral changes

349 was not due to the stimulation parameters. Analysis of lines containing both $\mathrm{pC} 1 \mathrm{~d}$ and $\mathrm{pC} 1 \mathrm{e}$

350 exhibited similar levels of behavior to containing pC1d alone, implying that $\mathrm{pC} 1 \mathrm{~d}$ and $\mathrm{pC} 1 \mathrm{e}$ do

351 not act synergistically (Figure 5-figure supplement $4 \mathrm{~A}-\mathrm{B}$ ). Likewise, activation of $\mathrm{pC} 1 \mathrm{a}-\mathrm{c}$ did

352 not change the percentage of flies displaying aggression (Figure 6-figure supplement 5A - C).

353 Taken together, our results suggest that $\mathrm{pC} 1 \mathrm{~d}$, but not $\mathrm{pCle}$ or $\mathrm{pCla}-\mathrm{c}$, acts as a significant

354 facilitator of female aggression.
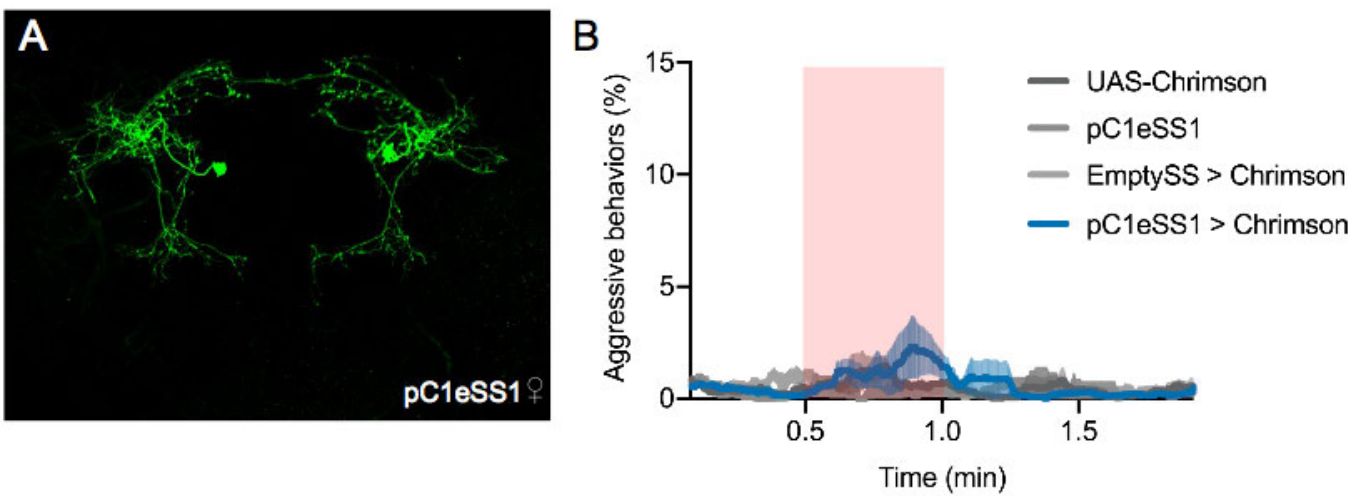

Figure 6. pC1e does not significantly increases aggressive social interactions in female flies.

(A) Maximum intensity projection (63x) image of the central brain of a female from the pCleSS1 line crossed with 20xUAS-CsChrimson::mVenus and stained with anti-GFP antibody. Images of the complete brain and ventral nerve cord of a female and male of the same genotype are shown in Figure 6-figure supplement 1A - C. (B) Percentage of flies engaging in aggressive behaviors over the course of a 2-minute trial during which a 30 -second $0.4 \mathrm{~mW} / \mathrm{mm}^{2}$ continuous light stimulus was delivered. Red shading indicates the stimulus period. The mean is represented as a solid line and shaded error bars represent variation between experiments. Each experiment included approximately 15 flies. 20xUAS-CsChrimson, $\mathrm{n}=3$ experiments; $\mathrm{pCleSS1}, \mathrm{n}=2$ experiments; EmptySS $>$ 20xUAS-CsChrimson, $\mathrm{n}=3$ experiments; $\mathrm{pC} 1 \mathrm{eSS} 1>20 \mathrm{xUAS-CsChrimson,} \mathrm{n}=3$ experiments. 
aIPg and pC1d neurons form reciprocal connections and only share a few overlapping preor post-synaptic connections

The generation of the full adult female brain (FAFB) electron microscopic (EM) image set

360 (Zheng et al., 2018) and the connectome of the hemibrain (Scheffer et al., 2020) allowed us to

361 use EM level connectomics to determine the structure of the circuit(s) that contained the cells

362 identified through our behavioral studies. First, we identified the cells in EM volumes that

363 correspond to those observed in our aIPg split-GAL4 lines. We began this work in FAFB, before

364 the availability of the hemibrain dataset. First, the fiber bundle in each hemisphere that contained

365 the neurons corresponding to the $\sim 32$ cells of the aIP-g lineage based on cell body and soma tract

366 position in the brain (Cachero et al., 2010) was identified. We sufficiently traced the axon and

367 major dendritic arbors of all these cells to determine if they matched the morphologies of the

368 neurons observed in our split-GAL4 lines (Figure 7). While the hemibrain connectome was being

369 generated, we were able to identify the corresponding aIPg cells (Figure 7 - figure supplement 1)

370 as well as those of the $\mathrm{pC} 1$ cluster, allowing us to participate in the effort to improve the

371 accuracy of their reconstruction and analyze their morphology in greater detail. The results of

372 these reconstructions are in the v1.0 connectome (neuprint.janelia.org) reported in Scheffer et al.

373 (2020). Based on morphological differences, most notably in their SMP and SIP arbors, and later

374 confirmed by their distinct connectivity, we separated the aIPg neurons into 3 distinct types, aIPg

375 type 1, aIPg type 2, and aIPg type 3 (Figure 8 and Videos 7 and 8). Light microscopy images

376 confirmed the presence of these aIPg types within our split-GAL4 lines (Figure 1- figure

377 supplement 2). 
bioRxiv preprint doi: https://doi.org/10.1101/2020.05.27.118810; this version posted May 27, 2020. The copyright holder for this preprint (which

was not certified by peer review) is the author/funder, who has granted bioRxiv a license to display the preprint in perpetuity. It is made available under aCC-BY 4.0 International license.

A
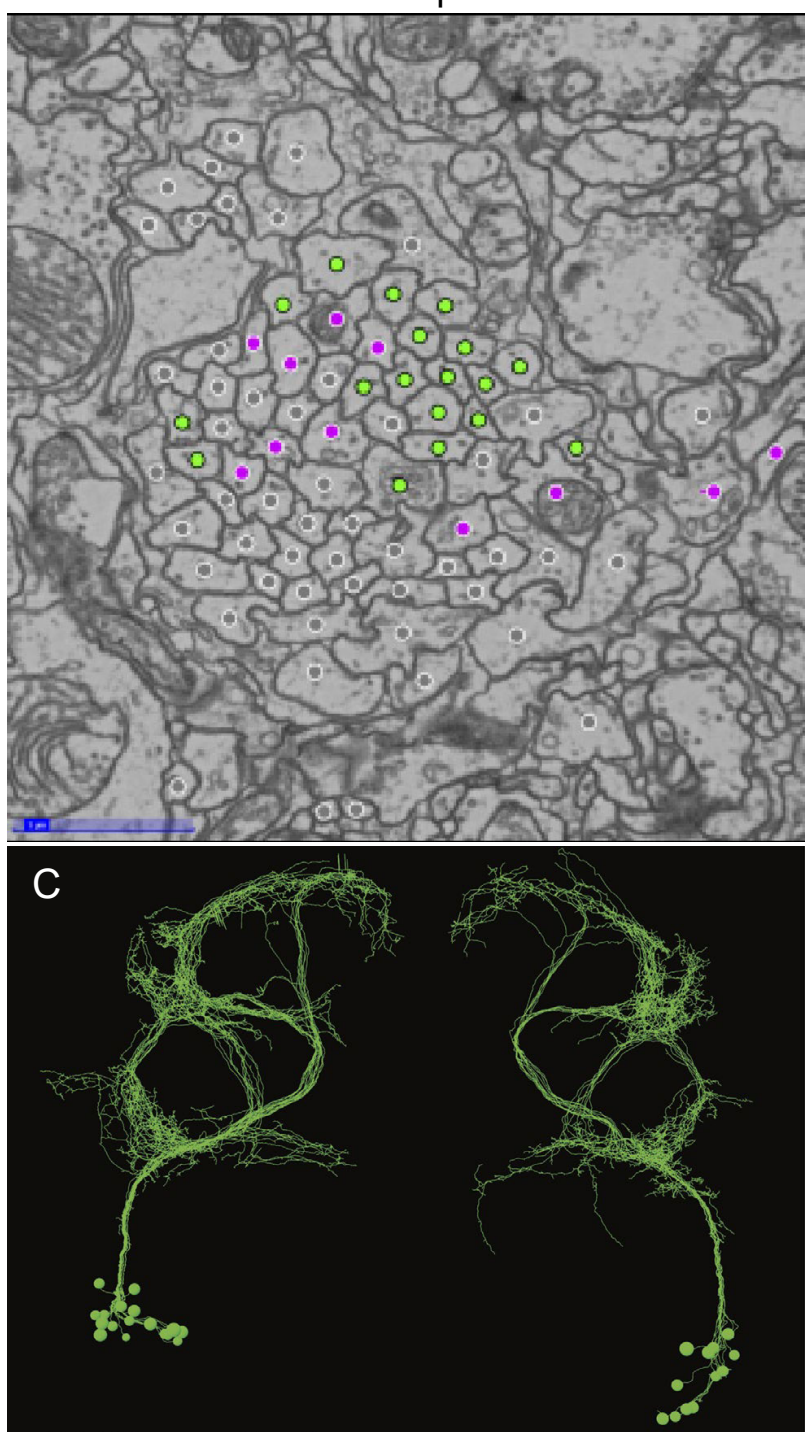

B

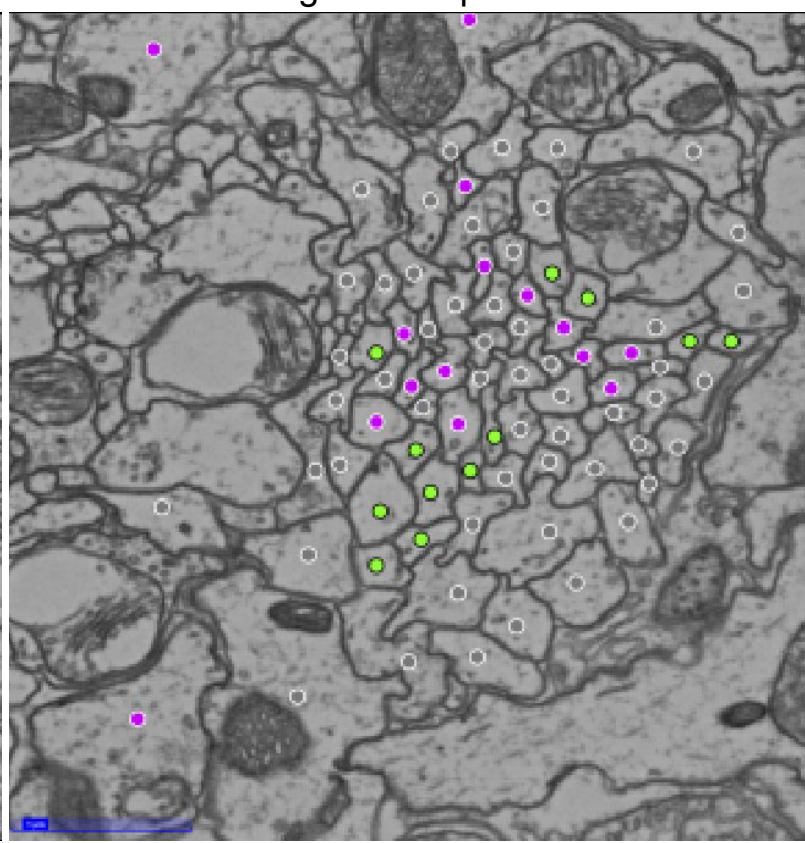

D

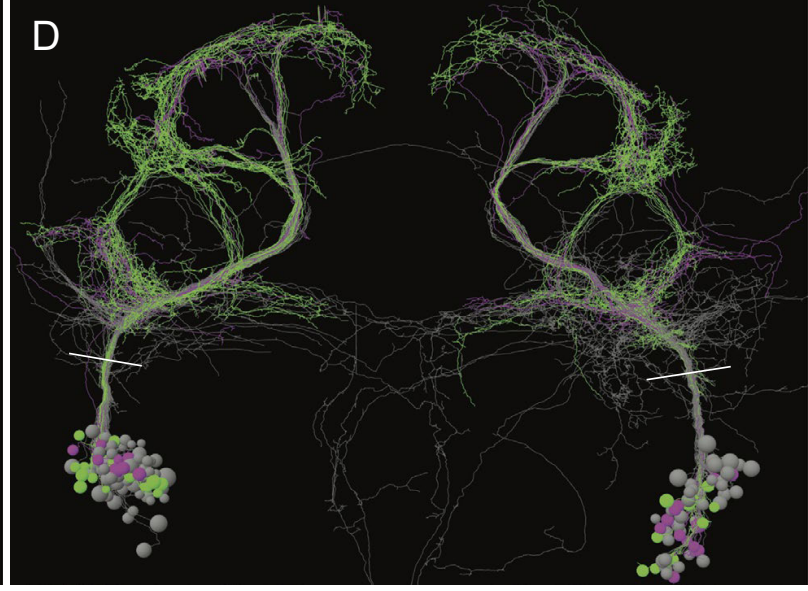

clearly different

Figure 7. Identification of aIPg neurons in the FAFB dataset.

(A - B) Images of an area of the left and right hemisphere of an EM section from the FAFB dataset containing the fiber tracts of the 32 putative aIP-g neurons described by Cachero et al. (2010). A dot has been placed in each axon, color-coded to reflect the degree of similarity of its morphology, revealed by manual tracing and visual inspection, to the aIPg neurons contained in our split-GAL4 lines. Grey represents neurons whose morphology clearly differed, magenta represents neurons whose morphology were similar but differed in one or more branches, and green represents neurons that we judged to correspond to those in our split-GAL4 lines. Note that, as we often observe in our split-GAL4 lines (see Figure 1-figure supplement 1), the number of neurons differed between hemispheres; in this case, the left hemisphere had 17 green cells, while the right hemisphere had only 12. (C - D) Skeleton rendering of the traced aIPg neurons, colored based on their similarity to the aIPg neurons identified in our split-GAL4 lines. Panel C shows only cells we judged to correspond to those in our split-GAL4 lines, while D shows all traced cells. White lines in $\mathrm{D}$ indicate the approximate plane of $\mathrm{A}-\mathrm{B}$. Tracing of grey neurons was stopped when it was clear they did not match our split-GAL4 lines; therefore, their arbors are likely to be incomplete in these images. 
A
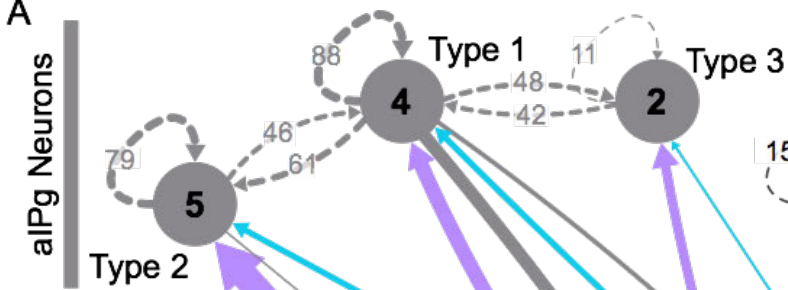

\section{3}

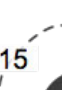

3 PVL04om/lm

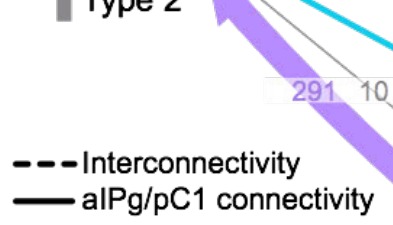

号
을
$\frac{0}{0}$

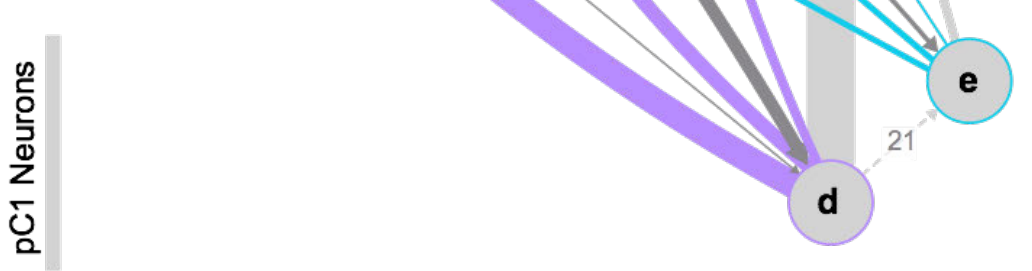

B

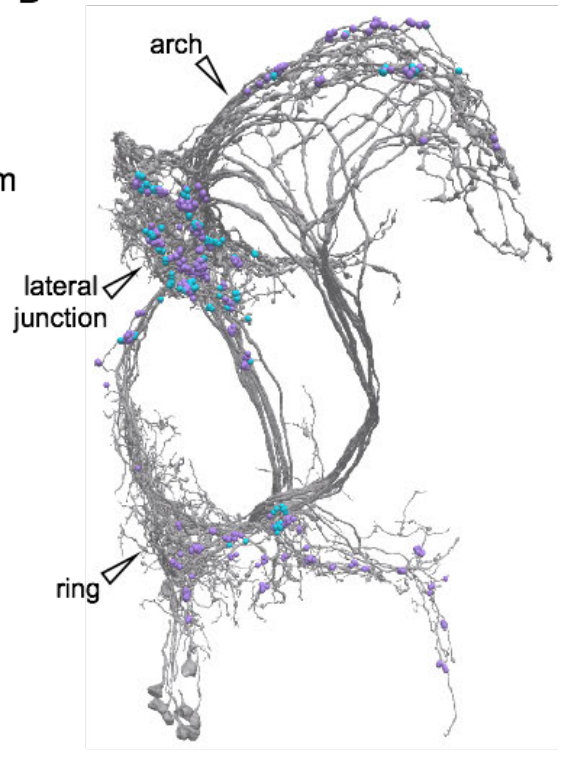

Figure 8. Reciprocal interactions between aIPg and pC1 neurons identified by EM connectomics.

(A) Interconnectivity between $\mathrm{pC} 1 \mathrm{~d}$, pC1e, aIPg Type 1, aIPg Type 2, aIPg Type 3, and PVL04om/lm neurons thresholded at 10 synapses. PVL04om/lm neurons also derive from the aIP-g lineage. Synapse number is noted on each arrow and dashed lines represent interconnectivity within the aIPg Type 1, Type 2 or Type 3, PVL04om/lm or $\mathrm{pC} 1$ neurons. Number within circle represents the number of neurons within the cell type; circles without numbers have only one neuron per brain hemisphere. Colored arrows depict synapses from pCld (purple) and pCle (blue) to aIPg types. (B) Electron microscopy rendering of all 11 Type 1, 2 and 3 aIPg neurons seen in the hemibrain with color-coded dots at the sites of synapses from pCld (purple) and pCle (blue); these synapses are concentrated in the lateral junction above the peduncle of the mushroom body. See Video 7 for more detail on interconnectivity between aIPg neuron types and Video 8 for detail on the connections between aIPg and $\mathrm{pC} 1$ neurons.

379 From analyzing the connectivity of aIPg neurons, we identified two pC1 cell types, pC1d and

380 pCle, among their top six presynaptic inputs. We are confident that these $\mathrm{pC} 1$ cells correspond

381 to those observed in our split-GAL4 lines (Figure 5- figure supplement 2, Figure 6-figure

382 supplement 2) as no other cells with similar morphology were found in the hemibrain volume.

383 These light to EM level assignments are also consistent with those reported by Wang et al (2020)

384 for light microscopy-FAFB correspondences. No connections above the threshold of 10 synapses

385 were identified in the hemibrain between aIPg neurons and the other three cell types in the $\mathrm{pC} 1$

386 cluster, pC1a - c (Figure 8-figure supplement 1). 
388 Another group of cells in the hemibrain volume, PVL04om/lm, is also heavily innervated by

389 pC1d (Figure 8A). These cells bear a morphological resemblance to aIPg neurons, but they have

390 distinct projections in the SMP. Moreover, PVL04om/lm neurons do not directly connect with

391 the aIPg type 1, type 2, or type 3 neurons and two of their top inputs include pC1a and pC1c,

392 which do not provide input to the aIPg neurons (Figure 8A and Figure 8-figure supplement 1).

393 Analysis of our split-GAL4 lines indicates that PVL04om/lm cells are unlikely to be consistently

394 present, especially in split-GAL4 lines, such as aIPgSS3, that have expression in a smaller

395 number of cells. Consistent with this interpretation, we have not observed PVL04om/lm cells in

396 our MultiColor FlpOut (MCFO) analyses of the aIPg lines. Their similar morphology combined

397 with the inherent variability of the cell number in our split-GAL4 lines, even between the brain

398 hemispheres of a single individual, means that we cannot formally rule out the possibility of

$399 \mathrm{PVL}$ 04om/lm cells contributing to activation phenotypes seen with our aIPg split-GAL4 lines.

400 Nevertheless, PVL04om/1m neurons were not included in further analysis and examination of the 401 role of these cells awaits split-GAL4 lines that specifically express in them.

402

403 Type 1 aIPg neurons provide strong reciprocal feedback onto pC1d (Figure 8A - B and Video 9),

404 but types 2 and 3 do not. There are also extensive reciprocal connections between aIPg neurons

405 of types 1 and 2 and between types 1 and 3, but not between types 2 and 3 (Figure $8 \mathrm{~A}$ and Video

406 8). 
A

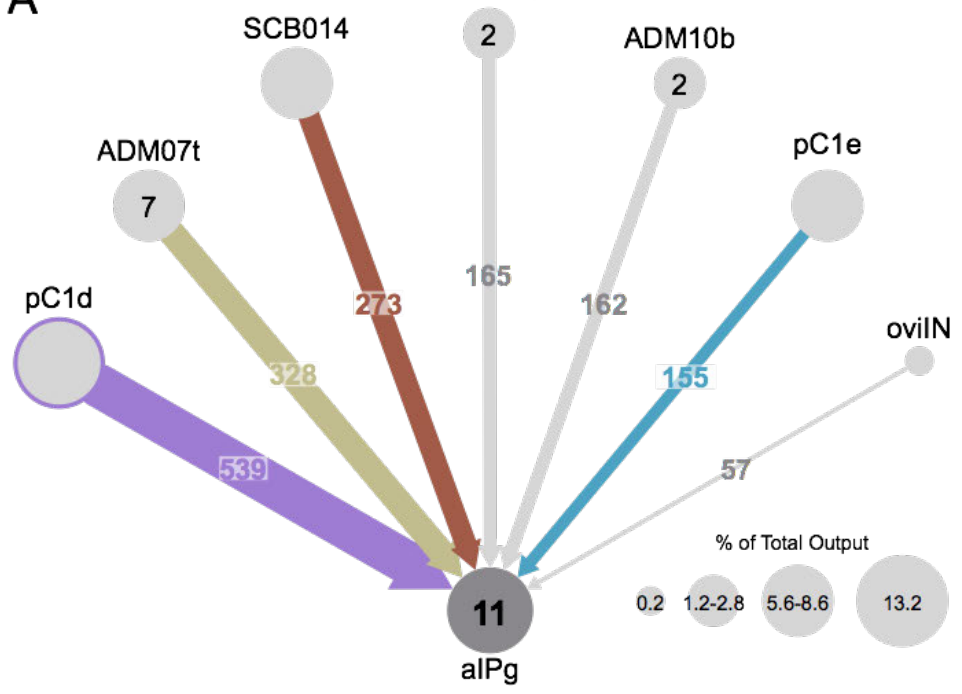

C

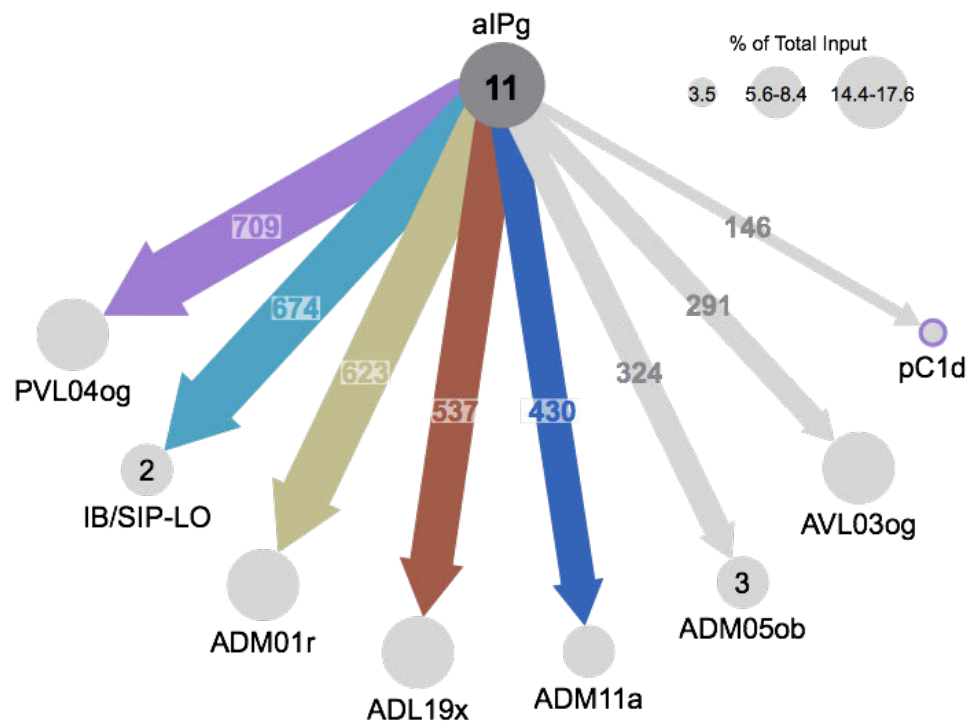

B

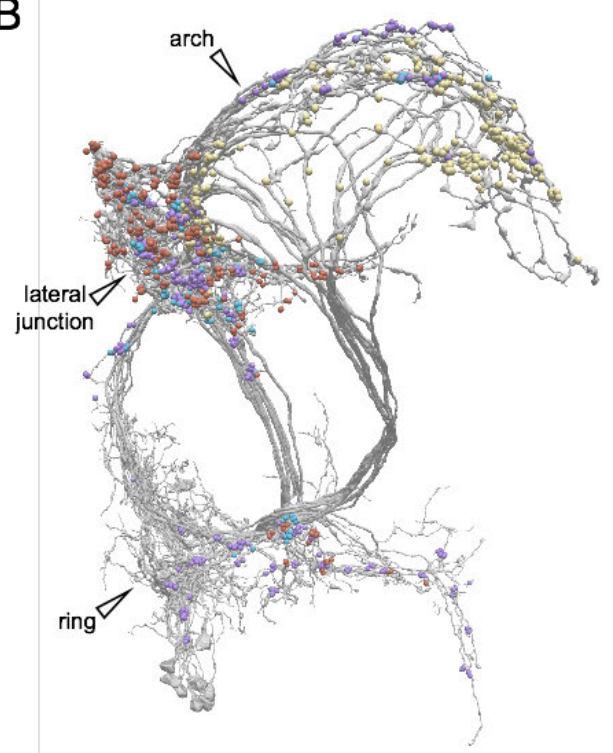

D

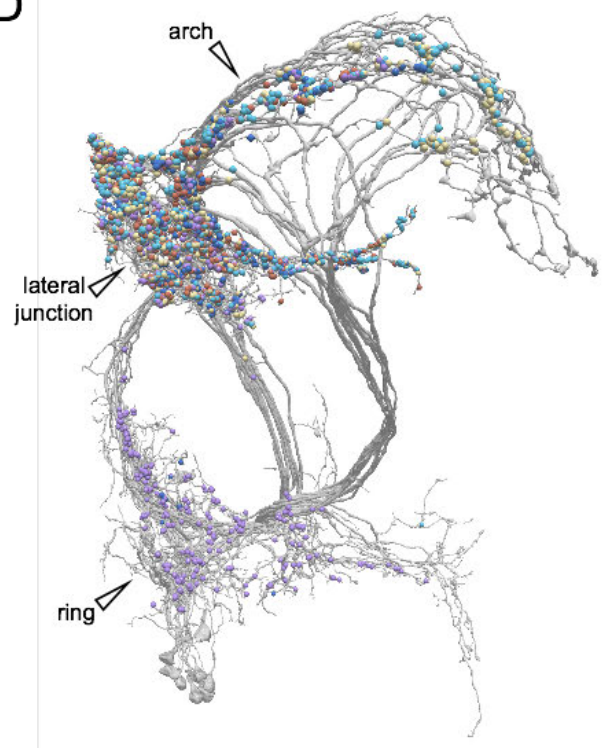

Figure 9. Major inputs to and outputs from aIPg neurons.

(A) Inputs to the 11 aIPg neurons in the right hemisphere thresholded at 150 synapses (inputs to all 11 aIPg neurons pooled). OviIN was included due to its involvement in other female behaviors. The size of the circles representing input neurons indicates the percentage of their output (estimated by synapse number) that goes to aIPg neurons. (B) Positions on the aIPg arbors of post-synaptic sites where these connections occur, color coded to match diagram in (A). While SCB014 forms a large number of synapses to all aIPg neuron types in the lateral junction, the majority of the synapses from the ADM07t neurons are on type 1 and 3 neurons and occur within the arch of the lateral protocerebral complex (LPC). (C) Post-synaptic outputs of aIPg neurons, thresholded at 290 synapses; pC1d at 146 synapses is included to show reciprocal connections. The size of the circles representing the downstream targets of aIPg indicates the percentage of their input (estimated by synapse number) that comes from aIPg neurons. (D) Positions on the aIPg arbors of the presynaptic sites where these connections occur are shown, color coded to match diagram in (C). See Video 9 for details on the inputs to aIPg neurons and Video 10 for details on their outputs. 
408 Among the aIPg neurons' six strongest pre-synaptic inputs, as judged by synapse number, we

409 found that $\mathrm{pC} 1 \mathrm{~d}$ and $\mathrm{pC} 1 \mathrm{e}$ rank first and sixth, respectively (Figure 9 and Video 10). A neuron

410 previously implicated in oviposition, oviIN (Wang et al. 2020), ranked eleventh among aIPg

411 inputs, based on synapses number (Figure 9 and Supplementary Table 2). The second and third

412 strongest inputs, SCB014 and the ADM07t, also provide presynaptic input to $\mathrm{pC} 1 \mathrm{~d}$, although it is

413 a lower percentage of their total output at 1.89\% from SCB014 and $0.22 \%$ from the ADM07t

414 group to $\mathrm{pC} 1 \mathrm{~d}$, compared to $7.7 \%$ and $8.6 \%$ to the aIPg neurons, respectively (Figure 9A, Video

415 10, Supplementary Table 2 and 3). OviIN also forms connections with both aIPg and pC1d, but

416 aside from these three cell types aIPg and $\mathrm{pC} 1 \mathrm{~d}$ do not share any strong pre-synaptic inputs

417 (Figure 9A - B, 10A - B, Video 10 and 12; note that ADM07t falls below the cutoff for being

418 displayed and for being considered a significant connection in Figure 10A and in Supplementary

419 Table 3). Only one of the top downstream targets of the aIPg neurons, ADM01r, is also a major

420 downstream target of pC1d (Figure 9C - D and 10C - D, Video 11 and 13, Supplemental Table 2

421 and 3). However, again we found that the connectivity strengths differ considerably: $1.13 \%$ of

422 ADM01r's input comes from pC1d, while $14.42 \%$ is provided by aIPg neurons (Figure 9C and

423 10C, Video 11, Supplemental Table 2 - 3). 

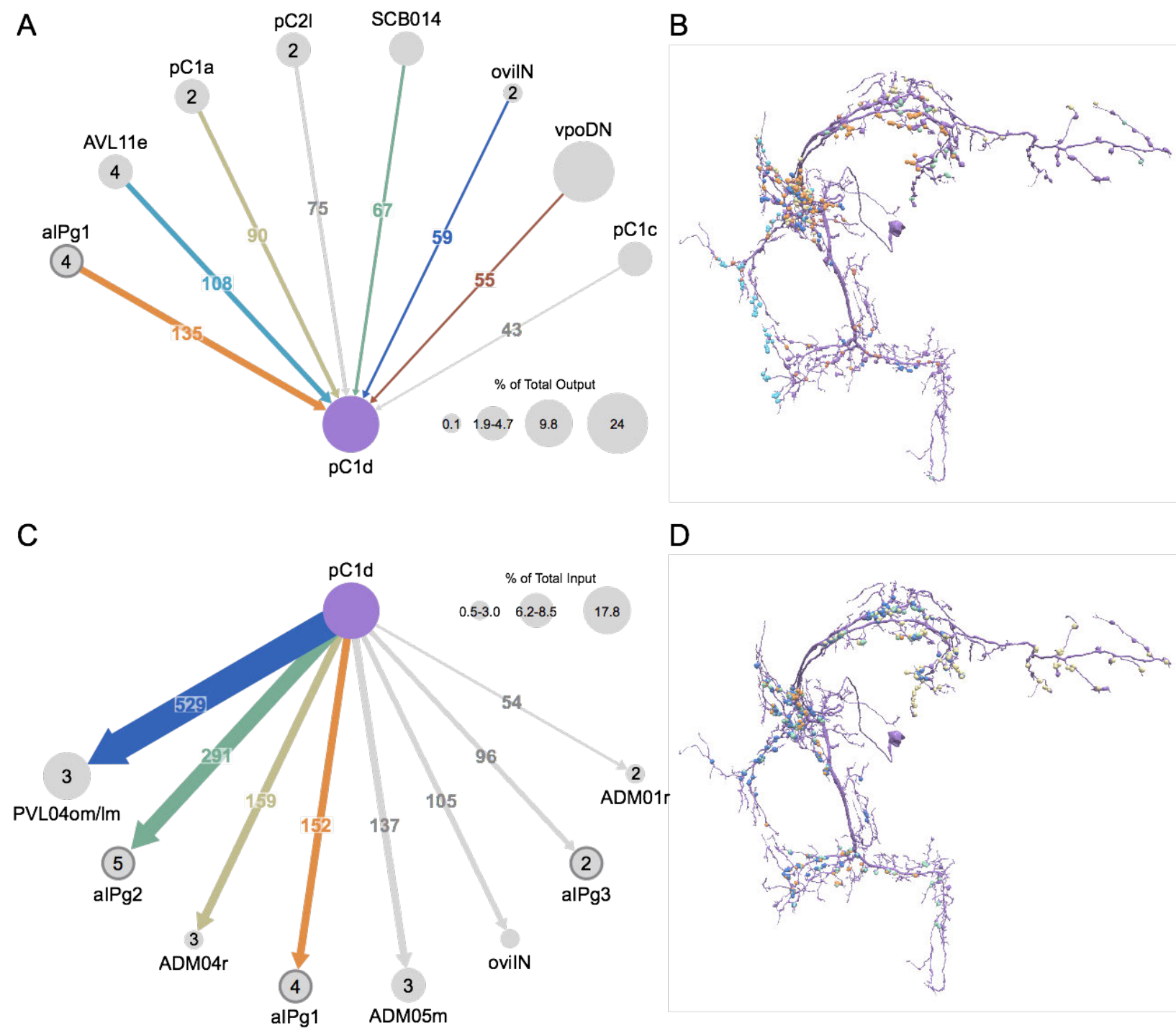

D

Figure 10. Major inputs to and outputs from pC1d.

(A) Inputs to the single right-hemisphere $\mathrm{pC} 1 \mathrm{~d}$ neurons thresholded at 90 synapses with other neurons of interest included. The size of the circles representing input neurons indicates the percentage of their output (estimated by synapse number in the hemibrain volume) that goes to $\mathrm{pCld}$; note that vpoDN is a descending interneuron with most of its outputs likely to lie outside the volume. aIPg neurons are indicated with a dark grey outline. (B) Positions on the $\mathrm{pC} 1 \mathrm{~d}$ arbor of post-synaptic sites, color coded to match diagram in (A). (C) Post-synaptic outputs of pC1d thresholded at 90 synapses with other neurons of interest included. The size of the circles representing output neurons indicates the percentage of their input (estimated by synapse number) that comes from $\mathrm{pC} 1 \mathrm{~d}$. aIPg neurons are indicated with a dark grey outline. (D) Positions on the $\mathrm{pCld}$ arbors of the presynaptic sites where these connections occur are shown, color coded to match diagram in (C). See Video 11 for details on the inputs to pC1d and Video 12 for details on pC1d's outputs.

425 Inputs to pC1d include several cell types implicated in oviposition and receptivity (Wang et al., 
central brain as judged by synapse number (Figure 7A, Supplemental Table 3) (Wang et al.

separated sites on the pC1d arbor (Figure 10B and Video 12), suggesting a potential spatial

separation of the circuit connections underlying these two social behaviors. These connections

may play a role in the flies' ability to display one action at a time.

\section{Discussion}

435 The circuits that govern aggression in Drosophila are known to be sexually dimorphic and are

436 poorly understood in females. In this paper, we described female aggressive behaviors, uncover

437 key components of the underlying neuronal circuits, develop genetic reagents to manipulate

438 these neurons, and map their connections using EM-level connectomics. Specifically, we

439 discovered the involvement of a subset of the aIPg lineage, a collection of cell types not

440 previously implicated in social behaviors, in mediating female aggressive social interactions.

441 Optogenetic activation with the channelrhodopsin CsChrimson dramatically increased aggression

442 in lines labelling this subset of aIPg neurons, while inactivation diminished these actions.

443 Analysis using EM-level connectomics revealed strong connectivity between these aIPg neurons

444 and two members of the $\mathrm{pC} 1$ cluster, a group of related cell types previously linked with social

445 behaviors (Hoopfer et al., 2015; Palavicino-Maggio et al., 2019; Scheffer et al., 2020; Wang et

446 al., 2020; Zhou et al., 2014). In particular, pC1d is the top pre-synaptic input to aIPg neurons,

447 devoting $\sim 13 \%$ of its output synapses to them. Behavioral tests using split-GAL4 lines cleanly

448 labeling $\mathrm{pC} 1 \mathrm{~d}$ demonstrated its ability to increase female aggression. In contrast, we found no

449 evidence for the involvement of the other four $\mathrm{pC} 1$ cell types, $\mathrm{pC} 1 \mathrm{a}-\mathrm{c}$ and $\mathrm{pC} 1 \mathrm{e}$, in aggression;

450 however, we found that $\mathrm{pC} 1 \mathrm{e}$ makes synapses onto aIPg neurons raising the possibility that this

451 cell type plays a role that was not revealed by our behavioral assays. 
bioRxiv preprint doi: https://doi.org/10.1101/2020.05.27.118810; this version posted May 27, 2020. The copyright holder for this preprint (which was not certified by peer review) is the author/funder, who has granted bioRxiv a license to display the preprint in perpetuity. It is made available under aCC-BY 4.0 International license.

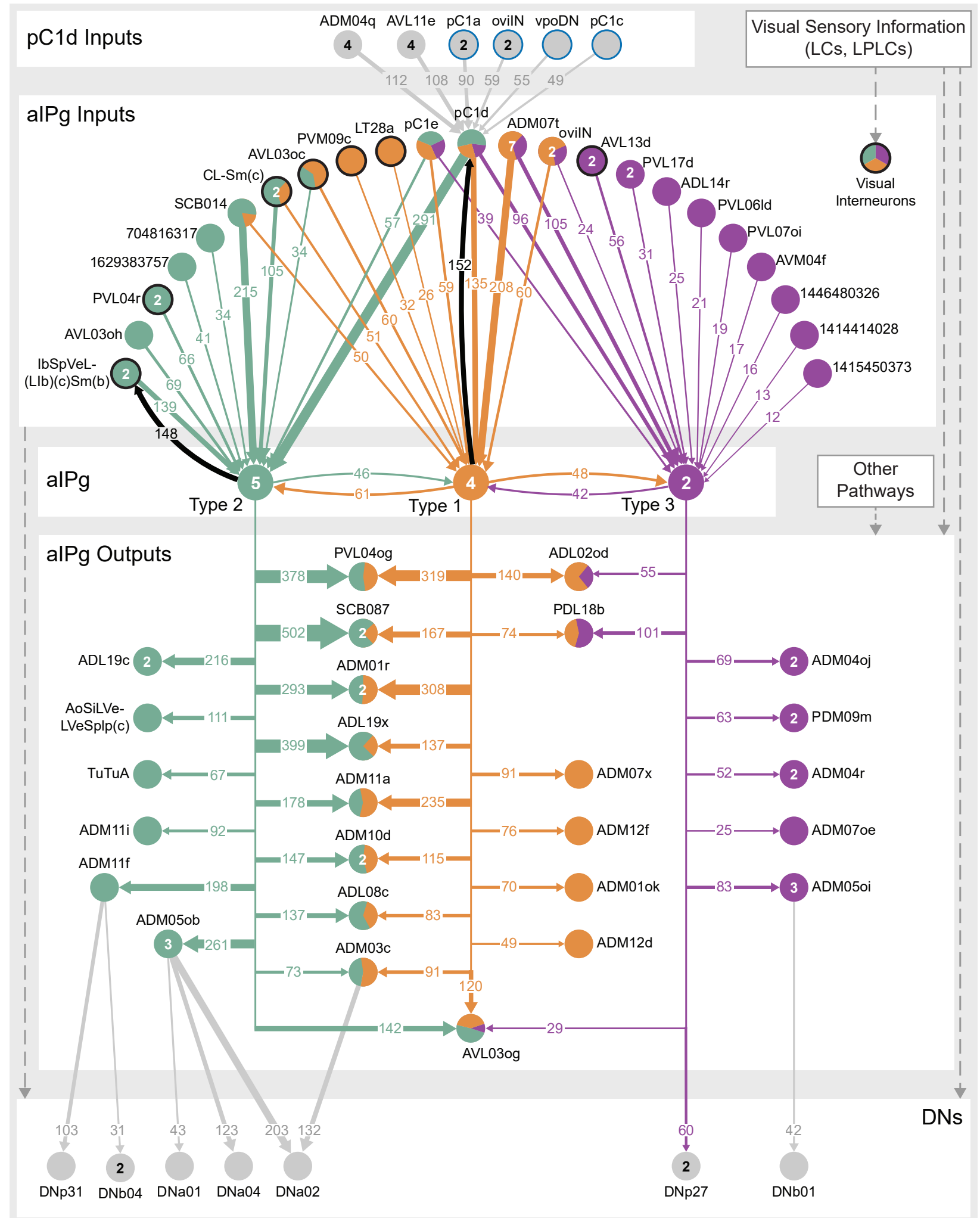

Figure 11. Proposed circuit underlying female aggressive behaviors.

A circuit diagram showing the key neuronal pathways we uncovered in our behavioral and connectomics analyses. The numbers in arrows represent synapse number. We propose that $\mathrm{pC} 1 \mathrm{~d}$ facilitates female aggressive behaviors through aIPg type 1 (orange), type 2 (green), and type 3 neurons (purple), which act as a mediator of these social 
interactions. The aIPg type 1 neurons, based on their connections with the other two types and feedback onto pC1d, appear to be important for the recurrency within the circuit. We propose that visual information enters the circuit through interneurons (black outline) that innervate aIPg neurons (see Figure 11-figure supplement 1) or at later nodes in the circuit. Like pC1d, other neuronal populations (color-coded by the proportion of their synapses on each aIPg type) also provide different proportions of their output to the aIPg type 1, aIPg type 2 and aIPg type 3 subtypes. The EM dataset further identified differences in the post-synaptic targets of aIPg type 1, aIPg type 2 and aIPg type 3 neurons, which are shown color-coded by the relative proportion of their synaptic input they receive from each aIPg type. Additionally, while only aIPg type 3 neurons directly connect with a descending interneuron (DN), we identified four post-synaptic targets of the aIPg type 1, aIPg type 2 and aIPg type 3 neurons that form synapses onto DNs, which may function as motor outputs of the circuit. The top four inputs to $\mathrm{pCld}$ and three other neurons of interest are shown. These include neurons implicated in other female behaviors (blue outline), including oviposition (oviIN, pC1a) and mating (vpoDN), suggesting communication between the circuits underlying these interactions. Inputs to the aIPg neurons were thresholded at 6 synapses per aIPg neuron, resulting in collective thresholds of 24 , 30 , and 12 synapses to aIPg type 1 , aIPg type 2 , and aIPg type 3, respectively. Outputs of the aIPg neurons were similarly adjusted based on the aIPg type, with 48,60 , and 24 synapses used as the collective thresholds for aIPg type 1, aIPg type 2, and aIPg type 3, respectively. Connections with DNs were thresholded at 30 synapses. Connections between IbSpVeL-(LIb)(c)Sm(b), a strong reciprocal target of aIPg type 2, and DNa03 (334 synapses) and DNb01 (32 synapses), as well as connections between pC1d and DNa01 (40 synapses) are not shown.

\section{aIPg neurons mediate female aggressive behaviors}

454 Innate behaviors, including aggression, have been proposed to result from the interplay of

external stimuli and the internal state of the animal (Lorenz, 1963; Tinbergen, 1951). Neuronal

populations that have the ability to bypass normally required sensory cues to induce aggressive

457 behavior have been previously identified by experimentally activating select subsets of neurons

458 in Drosophila. For example, male-specific neurons expressing Drosophila tachykinin (DTK) act

459 as a hub mediating aggressive behaviors when activated and have been proposed to encode

460 higher levels of motivation (Anderson, 2016; Asahina, 2017; Asahina et al., 2014; Hashikawa et

461 al., 2018, 2017; Hoopfer, 2016). As aIPg appears to be both necessary and sufficient for

462 performing a high level of female aggression, we consider this population to be performing a

463 mediator function (Gregg, 2003; Siegel et al., 1999).

\section{4}

465 Neurons that provide input to aIPg could act as facilitators adjusting the degree of behavior or

466 conveying specific sensory information important for its initiation and execution. For example,

467 pC1d activation was able to drive aggressive behaviors but was not essential for these social 
interactions, indicating it may act as a facilitator. Stimulation of $\mathrm{pC} 1 \mathrm{e}$ neurons alone did not

470 when activated with pC1d. However, multiple external factors required for aggression in wild-

471 type females were not assessed in our activation experiments. For example, diet and the presence

472 of yeast in the arena are known to be important factors determining the level of female

473 aggression (Ueda and Kidokoro, 2002). Experiments examining the influence of such conditions

474 on the circuit and behavioral dynamics will be needed to evaluate pC1e's potential role. As

475 circuits can perform more than one behavioral output, $\mathrm{pC} 1 \mathrm{e}$ and the rest of this circuit could also

476 be involved in other interactions that will need to be studied using additional assays.

\section{aIPg type 1, type2 and type 3 neurons differ in their pre- and post-synaptic connections}

479 The aIPg neurons we studied are three distinct cell types that differ in morphology and

480 connectivity. Our efforts to derive split-GAL4 lines specific for each of these cell types have

481 been unsuccessful, which has limited our ability to explore their individual roles. Nor have we

482 yet performed physiological experiments that might reveal distinct features of the responses of

483 these neurons when the circuit is activated. Nevertheless, we propose that the three aIPg types

484 found in our split-GAL4 lines make distinct contributions to the phenotypes we observed, as

485 there are many differences in their inputs and outputs (Figure 11). For example, while pC1d

486 makes strong connections onto all three aIPg types, it only receives strong recurrent feedback

487 from type 1 neurons. Similarly, SCB014, which is the third top input to the aIPg neurons, 488 predominately sends its projections to type 2 neurons. There are likewise many differences in 489 downstream targets, some of which connect to descending neurons (Figure 11). Generating split- 
490 GAL4 lines that specifically target such cell types will allow us to perform experiments to 491 explore their roles in aggressive behaviors.

\section{Activation of aIPg neurons generated a persistent internal state resulting in continued}

495 In both males and female flies, aggression-related behaviors have been found to extend beyond

496 the stimulation period of certain neuronal cell types (Deutsch et al., 2020; Hoopfer et al., 2015),

497 suggesting a persistent internal state. We did observe head butting and fencing behaviors after

498 the cessation of the optogenetic stimulus when lines labeling aIPg cells were used. Additionally,

499 the extent of these behaviors depended on stimulus conditions. In contrast, levels of post-

500 stimulus aggressive behavior similar to those observed in aIPg lines were not found when

501 activating $\mathrm{pC} 1 \mathrm{~d}$ or $\mathrm{pC} 1 \mathrm{e}$, or a combination of these two cell types. Persistence of aggression after

502 stimulating a combination of $\mathrm{pC} 1 \mathrm{~d}$ and $\mathrm{pC} 1 \mathrm{e}$ has been reported following a 5 minute stimulation

503 paradigm (Deutsch et al., 2020) rather than the 30 second stimulation we used. It is possible that

504 such persistence is the result of downstream activation of aIPg neurons. However, it is important

505 to keep in mind that the stimulus conditions required to induce persistent aggression are more

506 intense than those needed to generate high levels of aggression during the stimulus period itself

507 and the ethological relevance of persistence in female aggression is unclear. Female aggressive

508 bouts range from 10 to 30 seconds depending on their mated status as opposed to an average of

50980 seconds for single-housed males (Bath et al., 2017; Yuan et al., 2014). Additionally, female

510 flies do not appear to establish dominance, and aggressive behaviors are not found to escalate

511 over the course of a trial (Nilsen et al., 2004; Vrontou et al., 2006). While some persistence is

512 needed to maintain aggressive behaviors during bouts lasting tens of seconds, it is unclear what 
513 role longer persistence would play. Consistent with this view, we observed that aggressive

514 behaviors took only a few 10 s of seconds to return to baseline levels after the stimulation of aIPg

515 neurons.

517 Multiple recurrent circuit motifs that could provide the basis for persistent neural activity over

518 this time scale were revealed by our connectomics analyses. In particular, the feedback between

519 aIPg type 1 neurons and pC1d, and recurrent connections between the aIPg neurons themselves,

520 are obvious candidates as all of these neurons are cholinergic and thus putatively excitatory. The

521 higher levels of stimulation required to induce persistence raises the possibility that this

522 phenotype is an experimental artefact of non-physiological levels of stimulation of those same

523 circuits. Nevertheless, it seems likely that more subtle changes in brain state persist, and the

524 circuit mechanisms of such changes remain an open question. For example, our RNA profiling

525 experiments raise the possibility of a role for the neuropeptide sNPF.

527 Changes in brain state, such as those occurring during aggressive encounters, also influence

528 sensory processing. For example, olfactory cues paired with a male fly winning an aggressive

529 encounter become associated with reward (Kim et al., 2018). We observed that females quickly

530 altered their speed and direction following aIPg stimulation, appearing to approach other flies.

531 These behaviors raise the possibility that visual signals influence the "decision" to be aggressive.

532 For example, certain features of the target, including its size and distance, might be important to

533 drive aggression through aIPg neurons. Another non-exclusive hypothesis is that aggression

534 includes behaviors that rely on vision, such that aIPg activation recruits orientation circuits and

535 other pathways also used by different types of social interactions. Connections were identified in 
536 the hemibrain between visual interneurons and all three types of the aIPg neurons (Figure 11 and

537 Figure 11-figure supplement 1), which would be in a position to convey information about the

538 target and salient aspects of the environment. Specific types of lobular columnar cells convey

539 different information (Keleş and Frye, 2017; Wu et al., 2016), and these inputs differ amongst

540 the aIPg types. For example, optogenetic activation of LC10 can elicit behaviors resembling

541 reaching (Wu et al, 2016) and components of courtship behavior (Ribeiro et al., 2018), and these

542 neurons connect through an interneuron to aIPg type 1 and type 2. Additionally, visual

543 information could be integrated at later nodes within the circuit, as has been shown for P9

544 descending neurons involved in mediating courtship behavior (Bidaye et al., 2019).

Interactions between pC1d and neurons implicated in other female social behaviors

547 Our analysis of the pC1d and aIPg also revealed connections to neurons known to be involved in

548 other female-specific behaviors, including oviposition. Links between oviposition and female

549 aggression have been observed in field work on other Drosophila species, with increased

550 aggressive behaviors occurring on egg laying sites (Shelly, 1999). Supporting this close

551 relationship between the behaviors, $\mathrm{pC} 1 \mathrm{~d}$ receives innervation from $\mathrm{pC} 1 \mathrm{a}$, a cell type involved in

552 the egg laying pathway (Wang et al., 2020). Interestingly, Wang et al. (2020) noted differences

553 in the depolarization of $\mathrm{pC} 1 \mathrm{~d}$ and $\mathrm{pC} 1 \mathrm{e}$ following activation of the sex-peptide abdominal

554 ganglion (SAG) neuron in the sex-peptide pathway. While these are not as extensive as seen in

$555 \mathrm{pCla}$, they provide a potential neural basis for the reported effect of mating status on female

556 aggressive behavior (Bath et al., 2020, 2018; Ueda and Kidokoro, 2002). Further supporting the

557 close tie between these two social behaviors, we found that a very high proportion of the

558 synaptic output of vpoDN in the central brain goes to $\mathrm{pC} 1 \mathrm{~d}$ and oviIN innervates both $\mathrm{pC} 1 \mathrm{~d}$ and 
559 aIPg neurons (Wang et al., 2020a,b). Mating behavior and aggression also overlaps at the P1/pC1

560 level in male flies, raising the possibility of a common node in both sexes (Hoopfer et al., 2015;

561 Wang et al., 2020). Due to their connectivity, we expect aIPg, pC1d, and pC1e to be involved in

562 a range of social interactions in addition to aggression.

564 Concluding remarks

565 The female fly must integrate information from sensory cues about the external world—such as

566 the presence of other individuals, food and egg laying sites — with internal states — such as

567 hunger and mating status — when weighing when to initiate or abandon aggressive interactions.

568 Our work provides a foundation for future studies aimed at understanding the complex social

569 behavior of aggression by identifying key neuronal cell types, placing them in the context of a

$570 \quad$ larger neuronal circuit, and providing tools for their manipulation.

571

572

573

574

575

576

577

578

579

580

581

582

583

\section{Acknowledgements}

We thank Drs. U. Heberlein, D. J. Anderson, V. Chiu, K. Longden, K. Wang, F. Wang, A. Nern, and T. Wolff as well as the Janelia community for their helpful suggestions during the course of this work and their comments on the manuscript. We thank the Janelia Project Technical Resources team (led by Gudrun Ihrke) for assistance with the manual correction of automated fly trajectories (Rebecca Arruda and NC) and performing neuronal segmentation of confocal images (Claire Managan). We also thank the Quantitative Genomics facility at Janelia for performing RNA profiling of the aIPg, pC1d, and pC1e lines, M. Eddison and J. Simon for their help in setting up the inactivation experiments and Igor Siwanowicz and Wyatt Korff for their assistance with the high-speed videos. We thank the Fly Light team for generating the images of GAL4 expression patterns and the connectome annotation team for their help with proofreading. 


\section{Materials and Methods}

585

586

587

588

589

590

591

592

$$
\text { impTNT-HA; (6) (w-); UAS-TNT-E (Sweeney et al. 1995). }
$$

Table 1. Split-GAL4 lines

\section{Fly stocks}

\begin{tabular}{|c|c|c|c|}
\hline Name & Number & AD Parent & DBD Parent \\
\hline emptySS & emptySS & BPp65ADZp in attP40 & BPZpGDBD in attP2 \\
\hline alPgSS1 & SS36564 & VT064565-pBPp65ADZpUw in attP40 & VT043699-pBPZpGdbdUw in attp2 \\
\hline alPgSS2 & SS36551 & VT043699-pBPp65ADZpUw in attP40 & R11A07-pBPZpGdbdUw in attp2 \\
\hline alPgSS3 & SS32237 & R11A07-pBPp65ADZpUw in attP40 & VT043700-pBPZpGdbdUw in attp2 \\
\hline alPgSS4 & SS47478 & R72C11-pBPp65ADZpUw in VK00027 & R11A07-pBPZpGdbdUw in attP2 \\
\hline alPgSS5 & SS56964 & R11A07-pBPp65ADZpUw in attP40 & R72C11-pBPZpGdbdUw in attp2 \\
\hline pC1dSS1 & SS56987 & R35C10-pBPp65ADZpUw in JK73A & R71A09-pBPZpGdbdUw in attp2 \\
\hline pC1dSS2 & SS57598 & R71A09-pBPp65ADZpUw in JK73A & R26F09-pBPZpGdbdUw in attp2 \\
\hline pC1dSS3 & SS43274 & VT25602-pBPp65ADZpUw in attP40 & VT002064-pBPZpGdbdUw in attp2 \\
\hline pC1dSS4 & SS59331 & R35C10-pBPp65ADZpUw in JK73A & VT002063-pBPZpGdbdUw in attp2 \\
\hline pC1eSS1 & SS59336 & R35C10-pBPp65ADZpUw in JK73A & R60G08-pBPZpGdbdUw in attp2 \\
\hline pC1eSS2 & SS39313 & R60G08-pBPp65ADZpUw in attP40 & VT002063-pBPZpGdbdUw in attp2 \\
\hline pC1eSS3 & SS59433 & R60G08-pBPp65ADZpUw in attP40 & R35C10-pBPZpGdbdUw in attp2 \\
\hline pC1a-c & SS75230 & R18A05-pBPp65ADZpUw in attP40 & dsx-ZpGal4DBD/TM6B \\
\hline
\end{tabular}

All experiments used virgin female flies unless otherwise stated. Flies were reared on standard cornmeal molasses food at $25^{\circ} \mathrm{C}$ and $50 \%$ humidity. For optogenetic activation experiments, flies

were reared in the dark on standard food supplemented with retinal (Sigma-Aldrich, St. Louis, MO), $0.2 \mathrm{mM}$ all trans-retinal prior to eclosion and $0.4 \mathrm{mM}$ all trans-retinal post eclosion. Hemidriver lines were created using gateway cloning as previously described (Dionne et al. 2018).

In addition to the split-GAL4 lines (see Table 1), the following fly strains were used: (1)

20XUAS-CsChrimson-mVenus in attP18 (Klapoetke et al., 2014); (2) w; Enhancer1-ADp65

(attP40); Enhancer2-ZpGAL4DBD (attP2) referred to as EmptySS and is described previously

(Pfeiffer et al., 2010; Luan et al., 2006; Aso et al., 2014); (3) MCFO-1 (Nern et al., 2015) (4)

UAS-GFP: pJFRC2-10XUAS-IVS-mCD8::GFP in attP2 (Pfeiffer et al., 2010); (5) UAS- 


\section{Optogenetic activation behavioral testing}

603 Groups of 13 - 18 group-housed virgin female flies (5 - 10 days post-eclosion) were tested at

$60425^{\circ} \mathrm{C}$ and $50 \%$ relative humidity in a $127 \mathrm{~mm}$ circular arena with a center depth of $3.5 \mathrm{~mm}$ as

605 described previously (Robie et al. 2017, Wu et al. 2014). Flies were loaded into the arena using

606 an aspirator. For activation of neurons expressing CsChrimson, the arena was uniformly

607 illuminated with 617 nm LEDs (Red-Orange LUXEON Rebel LED - 122 lm; Luxeon Star LEDs,

608 Brantford, Canada) at the power density specified in the figure legend. Unless otherwise stated,

609 all trials were performed under white-light illumination. For each trial, flies were acclimatized to

610 the area for $30 \mathrm{~s}$ prior to the delivery of a single constant stimulus lasting $30 \mathrm{~s}$. Pulse stimulation

611 at $0.1 \mathrm{~mW} / \mathrm{mm}^{2}$ was given in $30 \mathrm{~s}$ intervals with an inter-stimulus interval of 30 to $60 \mathrm{~s}$. The

612 pulse width was kept constant at $10 \mathrm{~ms}$ while the pulse number and period varied. Videos were

613 recorded from above using a camera (ROHS 1.3 MP B and W Flea3 USB 3.0 Camera; Point

614 Grey, Richmond, Canada) with an $800 \mathrm{~nm}$ long pass filter (B and W filter; Schneider Optics,

615 Hauppauge, NY) at 30 frames per second and $1024 \times 1024$ pixel resolution. Flies were tracked

616 using Ctrax (Branson et al. 2009) followed by automated classification of behavior with JAABA

617 classifiers (see Robie et al. (2017) or Supplementary file 1 for performance summary). For

618 experiments examining individual behaviors, the identities of the fly tracks were manually

619 corrected using the FixErrors GUI available with Ctrax. To validate classifiers, we manually

620 scored a separate group of ground-truth videos for head butting and fencing using the 'ground-

621 truthing' mode in JAABA. Using the balanced random feature, set of randomly selected frames

622 from the ground-truth videos was manually labeled as positive or negative for the behavior of

623 interest on a frame-by-frame basis. 
625 Activation experiments detailed in Figure 3 and Figure 5 supplement 9 were performed in 16

$626 \mathrm{~mm}$ diameter $\mathrm{x} 12 \mathrm{~mm}$ high aggression chambers using same LEDs and camera setup as detailed

627 above. For these experiments, two flies (genotypes and sex specified in figure) were introduced

628 into the arena with an aspirator. Flies were acclimatized to the arena for $30 \mathrm{~s}$ prior to delivery of

629 a single constant stimulus lasting 30 s. Flies were tracked using the Caltech FlyTracker

630 (http://www.vision.caltech.edu/Tools/FlyTracker/) followed by automated classification of

631 behavior with a JAABA classifier for head butting and fencing behaviors (see Supplementary

632 file 1 for performance summary).

634 For high speed videos, two flies were loaded into an arena using cold anesthesia and an aspirator.

635 The arena was illuminated with a ring light and a LED gooseneck. Constant stimulation was

636 provided from above during the recording at $0.45 \mathrm{~mW} / \mathrm{mm}^{2}$ with a $625 \mathrm{~nm}$ light source. Videos

637 were recorded with a Photon Fastcam Mini camera at 1000 frames per second.

639 Receptivity assays were performed as detailed in Wang et al. (2020). Briefly, one virgin female

640 and one wild-type single housed virgin male were transferred into a $10 \mathrm{~mm}$ diameter x $2 \mathrm{~mm}$

641 height arena through aspiration. Flies were videotaped under white-light illumination for 30 min

642 and photostimulated at $0.4 \mathrm{~mW} / \mathrm{mm}^{2}$ as described by Wang et al. (2020). The copulation latency

643 was examined manually. 
646 Virgin female flies were group-housed at a density of $20-40$ females per vial at $22^{\circ} \mathrm{C}$ on

647 dextrose media (79 g agar, 275 g yeast, 520 g cornmeal, 1100 g Dextrose, $87.5 \mathrm{~mL} \mathrm{20 \%}$

648 Tegosept, $20 \mathrm{~mL}$ Proprionic Acid in $11000 \mathrm{~mL}$ of water) for $21-28$ days. Female flies were

649 single housed for $5-7$ days and subsequently starved on $1 \%$ agarose in water for $20-24$ hours

650 prior to testing. Rearing and housing were performed in a light cycling incubator set for 12/12 hr

651 light/dark cycle. All inactivation experiments were run at $25^{\circ} \mathrm{C}$ and $40 \%$ humidity and performed

652 with two female flies per arena.

653

654 Assays were performed in previously described acrylic multi-chamber aggression arenas

655 (Hoopfer et al. 2015, Kim et al. 2018, Asahina et al. 2014). Each circular arena measured $16 \mathrm{~mm}$

656 diameter x $12 \mathrm{~mm}$ high and was coated with Insect-a-Slip (Bioquip Products, Rancho

657 Dominguez, CA) on the walls and SurfaSil Siliconizing Fluid (Thermo Fisher Scientific,

658 Waltham, MA) on the clear acrylic top plate to confine the flies to the bottom plate. The floor of

659 the arenas was composed of a uniform layer ( 1 mm thick) of apple juice-agarose food $(2.5 \%$

$660(\mathrm{w} / \mathrm{v})$ sucrose and $2.25 \%(\mathrm{w} / \mathrm{v})$ agarose in apple juice) that contained a $\sim 1 \mathrm{~mm}$ spot of live yeast

661 (Fleischmann, Cincinnati, Ohio) placed in approximately the center of each arena. Flies were

662 illuminated from beneath with visible light and recorded with ambient overhead room lighting.

663 Flies were introduced into the chamber with gentle aspiration through a hole in the top plate and

664 allowed to acclimatize for $30 \mathrm{~s}-1 \mathrm{~min}$ prior to recording. For automated analysis, flies were

665 tracked using the Caltech FlyTracker (http://www.vision.caltech.edu/Tools/FlyTracker/)

666 followed by automated classification of behavior with a JAABA classifier for head butting and

667 fencing behaviors (see Supplementary file 1 for performance summary). For manual analysis,

668 videos were blinded and scored for head butting and fencing behaviors using JWatcher software 
669 (http://www.jwatcher.ucla.edu/). Data were reported for each pair tested for manually annotated

670 analysis.

\section{Immunohistochemistry and imaging}

673 Dissection and immunohistochemistry of fly brains were carried out as previously described

674 (Jenett et al., 2012, Aso et al. 2019). Each split-GAL4 line was crossed to the same Chrimson

675 effector used for behavioral analysis. Full step-by-step protocols can be found at

676 https://www.janelia.org/project-team/flylight/protocols.

678 For single cell labelling of aIPg neurons, we used the MultiColor FlpOut (MCFO) technique (Nern et al., 2015). For MultiColor FlpOut (MCFO) experiments, the MCFO stock was crossed to a split-GAL4 line. Flies were collected after eclosion, transferred to a new food vial and

681 incubated in a $37^{\circ} \mathrm{C}$ water bath for $20-25 \mathrm{~min}$. These flies were dissected and underwent wholemount immunohistochemistry and confocal imaging (Dolan et al. 2018). 


\section{Connectomics Analysis}

694 Data collection and processing is specified in Zheng et al. (2018) for the FAFB dataset and

695 Scheffer et al. (2020) for the hemibrain dataset. Hemibrain data was queried using NeuPrint and 696 v1.0 of the connectome (neuprint.janelia.org). Visualizations of neuronal morphologies from the

697 hemibrain dataset were generated in NeuTu. Thresholds were used in order to limit the number

698 of neurons in the figures to those connections with the most synapses. The specific thresholds

699 used are given in each figure. In Figures $9-11$, neurons of interest that fell below the thresholds

700 indicated were also included. A complete list of synaptic connections can be found in NeuPrint.

702 For anatomical videos made in Blender (https://www.blender.org/), neuron and ROI meshes

703 were pulled directly from DVID using a set of python scripts (https://github.com/connectome-

704 neuprint/neuVid). Adobe Premier Pro and Adobe Illustrator were used to add text and diagrams

705 to the videos, respectively. Recordings for the narrations were performed using Camtasia.

706

707 Statistics

708 No statistical methods were used to pre-determine sample size. Sample size was based on

709 previous literature in the field and experimenters were not blinded in most conditions as almost

710 all data acquisition and analysis were automated. However, inactivation experiments in which

711 manual quantification was performed were blinded. All are biological replicates and behavioral

712 data are representative of at least 2 independent trials per experiment.

714 For each experiment, the experimental and control flies were collected, treated and tested at the 715 same time. A Mann-Whitney $U$ test or Kruskal-Wallis test and Dunn's post hoc test was used 
716 for statistical analysis. Comparisons with more than one variant were first analyzed using two-

717 way ANOVA. All statistical analysis was performed using Prism Software (GraphPad, version

718 7). $\mathrm{P}$ values are indicated as follows: $* * * \mathrm{p}<0.0001 ; * * * \mathrm{p}<0.001 ; * * \mathrm{p}<0.01$; and $* \mathrm{p}<0.05$

719 See Supplementary file 5 for exact $p$-values for each figure.

721 For boxplots, lower and upper whiskers represent 1.5 $\times$ IQR of the lower and upper quartiles,

722 respectively; boxes indicate lower quartile, median, and upper quartile, from bottom to top.

723 When all points are shown, whiskers represent range and boxes indicate lower quartile, median, 724 and upper quartile, from bottom to top. Shaded error bars on graphs are presented as mean \pm 725 s.e.m.

726

727 Competing interests

728 The authors declare no competing interests. 


\section{References}

Albert DJ, Nanji N, Brayley KN, Madryga FJ. 1979. Hyperreactivity as well as mouse killing is induced by electrical stimulation of the lateral hypothalamus in the rat. Behav Neural Biol 27:59-71. doi:10.1016/s0163-1047(79)92756-0

Alekseyenko OV, Chan Y-B, Okaty BW, Chang Y, Dymecki SM, Kravitz EA. 2019. Serotonergic modulation of aggression in Drosophila involves GABAergic and cholinergic opposing pathways. Curr Biol 29:1-12. doi:10.1016/j.cub.2019.05.070

Alekseyenko OV, Chan Y-B, De La Paz Fernandez M, Bülow T, Pankratz MJ, Kravitz EA. 2014. Single serotonergic neurons that modulate aggression in Drosophila. Curr Biol 24:2700-2707. doi:10.1016/j.cub.2014.09.051

Anderson DJ. 2016. Circuit modules linking internal states and social behaviour in flies and mice. Nat Rev Neurosci 17:692-704. doi:10.1038/nrn.2016.125

Asahina K. 2018. Sex differences in Drosophila behavior: qualitative and quantitative dimorphism. Curr Opin Physiol 6:35-45. doi:10.1016/j.physbeh.2017.03.040

Asahina K. 2017. Neuromodulation and strategic action choice in Drosophila aggression. Annu Rev Neurosci 40:51-75. doi:10.1146/annurev-neuro-072116-031240

Asahina K, Watanabe K, Duistermars BJ, Hoopfer E, González CR, Eyjólfsdóttir EA, Perona P, Anderson DJ. 2014. Tachykinin-expressing neurons control male-specific aggressive arousal in Drosophila. Cell 156:221-235. doi:10.1016/j.cell.2013.11.045

Aso Y, Sitaraman D, Ichinose T, Kaun KR, Vogt K, Belliart-Guérin G, Plaçais PY, Robie AA, Yamagata N, Schnaitmann C, Rowell WJ, Johnston RM, Ngo TTB, Chen N, Korff W, Nitabach MN, Heberlein U, Preat T, Branson KM, Tanimoto H, Rubin GM. 2014. Mushroom body output neurons encode valence and guide memory-based action selection in Drosophila. Elife 3:e4580. doi:10.7554/eLife.04580

Bandler RJJ, Chi CC, Flynn JP. 1972. Biting attack elicited by stimulation of the ventral midbrain tegmentum of cats. Science 177:364-366. doi:10.1126/science.177.4046.364

Bath E, Biscocho ER, Easton-Calabria A, Wigby S. 2020. Temporal and genetic variation in female aggression after mating. PLoS One 15: e0229633. doi:

10.1371/journal.pone.0229633

Bath E, Bowden S, Peters C, Reddy A, Tobias JA, Easton-Calabria E, Seddon N, Goodwin SF, Wigby S. 2017. Sperm and sex peptide stimulate aggression in female Drosophila. Nat Ecol Evol 1. doi:10.1038/s41559-017-0154

Bath E, Morimoto J, Wigby S. 2018. The developmental environment modulates mating-induced aggression and fighting success in adult female Drosophila. Funct Ecol 32:2542-2552. doi: $10.1111 / 1365-2435.13214$

Bellen HJ, Tong C, Tsuda H. 2010. 100 years of Drosophila research and its impact on vertebrate neuroscience: A history lesson for the future. Nat Rev Neurosci. 11: 514-522 doi:10.1038/nrn2839

Berntson GG. 1973. Attack, grooming, and threat elicited by stimulation of the pontine tegmentum in cats. Physiol Behav 11:81-87. doi:10.1016/0031-9384(73)90126-1

Bidaye SS, Laturney M, Chang AK, Liu Y, Bockemühl T, Büschges A, Scott K. 2019. Two brain pathways initiate distinct forward walking programs in Drosophila. bioRxiv https://doi.org/10.1101/798439.

Branson K, Robie AA, Bender J, Perona P, Dickinson MH. 2009. High-throughput ethomics in large groups of Drosophila. Nat Methods 6:451-457. doi:10.1038/nmeth.1328

Cachero S, Ostrovsky AD, Yu JY, Dickson BJ, Jefferis GSXE. 2010. Sexual dimorphism in the 
fly brain. Curr Biol 20:1589-1601. doi:10.1016/j.cub.2010.07.045

Chen P, Hong W. 2018. Neural circuit mechanisms of social behavior. Neuron. 98:16-30 doi:10.1016/j.neuron.2018.02.026

Chi CC, Flynn JP. 1971. Neural pathways associated with hypothalamically elicited attach behavior in cats. Science 171:703-706. doi:10.1126/science.171.3972.703

Deutsch D, Pacheco DA, Encarnacion-Rivera LJ, Pereira T, Fathy R, Calhoun A, Ireland EC, Burke AT, Dorkenwald S, McKellar C, Macrina T, Lu R, Lee K, Kemnitz N, Ih D, Castro M, Halageri A, Jordan C, Silversmith W, Wu J, Seung HS, Murthy M. 2020. The neural basis for a persistent internal state in Drosophila females. bioRxiv 2020.02.13.947952. doi:10.1101/2020.02.13.947952

Dickson BJ. 2008. Wired for sex: The neurobiology of Drosophila mating decisions. Science 322: 904-909 doi:10.1126/science. 1159276

Dierick HA, Greenspan RJ. 2007. Serotonin and neuropeptide F have opposite modulatory effects on fly aggression. Nat Genet 39:678-682. doi:10.1038/ng2029

Dionne H, Hibbard KL, Cavallaro A, Kao JC, Rubin GM. 2018. Genetic reagents for making split-GAL4 lines in Drosophila. Genetics 209:31-35. doi:10.1534/genetics.118.300682

Fathy R. 2016. Activation of central brain neurons in female Drosophila melanogaster promotes a persistent internal state that increases courtship receptivity and aggression. Princeton University, Senior thesis. http://arks.princeton.edu/ark:/88435/dsp01jw827f10m

Gregg TR. 2003. Cortical and limbic neural circuits mediating aggressive behavior. In: Mattson M.P. (ed) Neurobiology of Aggression Humana Press doi:10.1007/978-1-59259-382-8_1

Hashikawa K, Hashikawa Y, Lischinsky J, Lin D. 2018. The neural mechanisms of sexually dimorphic aggressive behaviors. Trends Genet. 30: 755-776 doi:10.1016/j.tig.2018.07.001

Hashikawa K, Hashikawa Y, Tremblay R, Zhang J, Feng JE, Sabol A, Piper WT, Lee H, Rudy B, Lin D. 2017. Esr1+ cells in the ventromedial hypothalamus control female aggression. Nat Neurosci 20:1580-1590. doi:10.1038/nn.4644

Hoffmann AA. 1990. The influence of age and experience with conspecifics on territorial behavior in Drosophila melanogaster. J Insect Behav 3:1-12. doi:10.1007/BF01049191

Hoopfer ED. 2016. Neural control of aggression in Drosophila. Curr Opin Neurobiol. 38: 109118 doi:10.1016/j.conb.2016.04.007

Hoopfer ED, Jung Y, Inagaki HK, Rubin GM, Anderson DJ. 2015. P1 interneurons promote a persistent internal state that enhances inter-male aggression in Drosophila. Elife 4. doi:10.7554/eLife. 11346

Hoyer SC, Eckart A, Herrel A, Zars T, Fischer SA, Hardie SL, Heisenberg M. 2008. Octopamine in male aggression of Drosophila. Curr Biol 18:159-167. doi:10.1016/j.cub.2007.12.052

Ishii K, Wohl M, DeSouza A, Asahina K. 2020. Sex-determining genes distinctly regulate courtship capability and target preference via sexually dimorphic neurons. Elife 9:1-34. doi:10.7554/eLife.52701

Keleş MF, Frye MA. 2017. Object-detecting neurons in Drosophila. Curr Biol 27:680-687. doi:10.1016/j.cub.2017.01.012

Kim SS, Franconville R, Turner-Evans D, Jayaraman V. 2015. Optogenetics in Drosophila melanogaster. In: Douglass A.D. (ed) New Techniques in Systems Neuroscience Springer International 147-176. doi:10.1007/978-3-319-12913-6_6

Kim YK, Saver M, Simon J, Kent CF, Shao L, Eddison M, Agrawal P, Texada M, Truman JW, Heberlein U. 2018. Repetitive aggressive encounters generate a long-lasting internal state in Drosophila melanogaster males. Proc Natl Acad Sci 115:1099-1104. 
doi:10.1073/pnas.1716612115

Klapoetke NC, Murata Y, Kim SS, Pulver SR, Birdsey-Benson A, Cho YK, Morimoto TK, Chuong AS, Carpenter EJ, Tian Z, Wang J, Xie Y, Yan Z, Zhang Y, Chow BY, Surek B, Melkonian M, Jayaraman V, Constantine-Paton M, Wong GKS, Boyden ES. 2014. Independent optical excitation of distinct neural populations. Nat Methods 11:338-346. doi:10.1038/nmeth.2836

Kravitz EA, Huber R. 2003. Aggression in invertebrates. Curr Opin Neurobiol. 13:736-743. doi:10.1016/j.conb.2003.10.003

Kruk MR, Van der Poel AM, Meelis W, Hermans J, Mostert PG, Mos J, Lohman AH. 1983. Discriminant analysis of the localization of aggression-inducing electrode placements in the hypothalamus of male rats. Brain Res 260:61-79. doi:10.1016/0006-8993(83)90764-3

Lammers JH, Kruk MR, Meelis W, van der Poel AM. 1988. Hypothalamic substrates for brain stimulation-induced attack, teeth-chattering and social grooming in the rat. Brain Res 449:311-327. doi:10.1016/0006-8993(88)91046-3

Lee H, Kim DW, Remedios R, Anthony TE, Chang A, Madisen L, Zeng H, Anderson DJ. 2014. Scalable control of mounting and attack by Esr1+ neurons in the ventromedial hypothalamus. Nature 509:627-632. doi:10.1038/nature13169

Lim RS, Eyjólfsdóttir E, Shin E, Perona P, Anderson DJ. 2014. How food controls aggression in Drosophila. PLoS One 9:e105626. doi:10.1371/journal.pone.0105626

Lorenz K. 1963. On aggression. Harcourt, Brace and World: New York. doi:10.4324/9780203994269

Luan H, Peabody NC, Vinson CRR, White BH. 2006. Refined spatial manipulation of neuronal function by combinatorial restriction of transgene expression. Neuron 52:425-436. doi:10.1016/j.neuron.2006.08.028

McKellar CE, Lillvis JL, Bath DE, Fitzgerald JE, Cannon JG, Simpson JH, Dickson BJ. 2019. Threshold-based ordering of sequential actions during Drosophila courtship. Curr Biol 29:426-434.e6. doi:10.1016/j.cub.2018.12.019

Nilsen SP, Chan Y-B, Huber R, Kravitz EA. 2004. Gender-selective patterns of aggressive behavior in Drosophila melanogaster. Proc Natl Acad Sci 101:12342-12347. doi:10.1073/pnas.0404693101

Nüsslein-Volhard C, Wieschaus E. 1980. Mutations affecting segment number and polarity in Drosophila. Nature 287:795-801. doi:10.1038/287795a0

Palavicino-Maggio CB, Chan YB, McKellar C, Kravitz EA. 2019. A small number of cholinergic neurons mediate hyperaggression in female Drosophila. Proc Natl Acad Sci 116:17029-17038. doi:10.1073/pnas.1907042116

Pavlou HJ, Goodwin SF. 2013. Courtship behavior in Drosophila melanogaster: Towards a “courtship connectome." Curr Opin Neurobiol. 23:76-83. doi:10.1016/j.conb.2012.09.002

Pfaff DW, Sakuma Y. 1979a. Deficit in the lordosis reflex of female rats caused by lesions in the ventromedial nucleus of the hypothalamus. J Physiol 288:203-210. doi:10.1113/jphysiol.1979.sp012691

Pfaff DW, Sakuma Y. 1979b. Facilitation of the lordosis reflex of female rats from the ventromedial nucleus of the hypothalamus. J Physiol 288:189-202. doi:10.1113/jphysiol.1979.sp012690

Pfeiffer BD, Ngo T-TB, Hibbard KL, Murphy C, Jenett A, Truman JW, Rubin GM. 2010. Refinement of tools for targeted gene expression in Drosophila. Genetics 186:735-55. doi:10.1534/genetics.110.119917 
Rezával C, Pattnaik S, Pavlou HJ, Nojima T, Brüggemeier B, D’Souza LAD, Dweck HKM, Goodwin SF. Activation of latent courtship circuitry in the brain of Drosophila females induces male-like behaviors. Curr Biol 26:76-83. doi:10.1016/j.cub.2016.07.021

Ribeiro IMA, Drews M, Bahl A, Machacek C, Borst A, Dickson BJ. 2018. Visual projection neurons mediating directed courtship in Drosophila. Cell 174:607-621.e18. doi:10.1016/j.cell.2018.06.020

Rideout EJ, Dornan AJ, Neville MC, Eadie S, Goodwin SF. 2010. Control of sexual differentiation and behavior by the doublesex gene in Drosophila melanogaster. Nat Neurosci 13:458-466. doi:10.1038/nn.2515

Robie AA, Hirokawa J, Edwards AW, Simpson JH, Reiser MB, Branson K, Umayam LA, Lee A, Phillips ML, Card GM, Korff W, Rubin GM. 2017. Mapping the neural substrates of behavior. Cell 170:393-406. doi:10.1016/j.cell.2017.06.032

Scheffer LK, Xu CS, Januszewski M, Lu Z, Takemura Shin-ya, Hayworth KJ, Huang G, Shinomiya K, Maitlin-Shepard J, Berg S, Clements J, Hubbard PM, Katz WT, Umayam L, Zhao T, Ackerman D, Blakely T, Bogovic J, Dolafi T, Kainmueller D, Kawase T, Khairy K, Leavitt L, Li PH, Lindsey L, Neubarth N, Olbris DJ, Otsuna H, Trautman ET, Ito M, Goldammer J, Wolff T, Svirskas R, Schlegel P, Neace E, Knecht C, Alvarado CX, Bailey DA, Ballinger S, Borycz JA, Canino BS, Cheatham N, Cook M, Dreher M, Duclos O, Eubanks B, Fairbanks K, Finley S, Forknall N, Francis A, Hopkins GP, Joyce EM, Kim S, Kirk NA, Kovalyak J, Lauchie S, Lohff A, Maldonado C, Manley EA, McLin S, Mooney C, Ndama M, Ogundeyi O, Okeoma N, Ordish C, Padilla N, Patrick CM, Paterson T, Phillips EE, Phillips EM, Rampally N, Ribeiro C, Robertson MK, Rymer JT, Ryan SM, Sammons M, Scott AK, Scott AL, Shinomiya A, Smith C, Smith K, Smith NL, Sobeski MA, Suleiman A, Swift J, Takemura Satoko, Talebi I, Tarnogorska D, Tenshaw E, Tokhi T, Walsh JJ, Yang T, Horne JA, Li F, Parekh R, Rivlin PK, Jayaraman V, Ito K, Saalfeld S, George R, Meinertzhagen I, Rubin GM, Hess HF, Jain V, Plaza SM. 2020. A connectome and analysis of the adult Drosophila central brain. bioRxiv 2020.04.07.030213.

Shelly TE. 1999. Defense of oviposition sites by female oriental fruit flies (Diptera: Tephritidae). Florida Entomol 82:339. doi:10.2307/3496587

Siegel A, Roeling TAP, Gregg TR, Kruk MR. 1999. Neuropharmacology of brain-stimulationevoked aggression. Neurosci Biobehav Rev 23:359-389. doi:10.1016/S01497634(98)00040-2

Simon JC, Dickinson MH. 2010. A new chamber for studying the behavior of Drosophila. PLoS One 5:e8793. doi:10.1371/journal.pone.0008793

Simpson JH, Looger LL. 2018. Functional imaging and optogenetics in Drosophila. Genetics 208:1291-1309. doi:10.1534/genetics.117.300228

Siwicki KK, Kravitz EA. 2009. Fruitless, doublesex and the genetics of social behavior in Drosophila melanogaster. Curr Opin Neurobiol. 19:200-206. doi:10.1016/j.conb.2009.04.001

Sturtevant AH. 1915. Experiments on sex recognition and the problem of sexual selection in Drosophilia. J Anim Behav. 5:351-366 doi:10.1037/h0074109

Sweeney ST, Broadie K, Keane J, Niemann H, O’Kane CJ. 1995. Targeted expression of tetanus toxin light chain in Drosophila specifically eliminates synaptic transmission and causes behavioral defects. Neuron 14:341-351. doi:10.1016/0896-6273(95)90290-2

Takahashi A, Miczek KA. 2015. Neurogenetics of aggressive behavior: Studies in rodents. Curr Top Behav Neurosci 17:3-44. doi:10.1007/7854_2013_263 
914

915

916

917

918

919

920

921

922

923

924

925

926

927

928

929

930

931

932

933

934

935

936

937

938

939

940

941

942

943

944

945

946

947

948

949

950

951

952

953

954

955

956

957

958

959
Tinbergen N. 1951. The study of instinct. New York, NY, US: Clarendon Press/Oxford University Press.

Tirian L, Dickson BJ. 2017. The VT GAL4, LexA, and split-GAL4 driver line collections for targeted expression in the Drosophila nervous system. bioRxiv 198648. doi:10.1101/198648

Ueda A, Kidokoro Y. 2002. Aggressive behaviours of female Drosophila melanogaster are influenced by their social experience and food resources. Physiol Entomol 27:21-28. doi:10.1046/j.1365-3032.2002.00262.x

Vrontou E, Nilsen SP, Demir E, Kravitz EA, Dickson BJ. 2006. fruitless regulates aggression and dominance in Drosophila. Nat Neurosci 9:1469-1471. doi:10.1038/nn1809

Wang F, Wang K, Forknall N, Patrick C, Yang T, Parekh R, Bock D, Dickson BJ. 2020a. Neural circuitry linking mating and egg laying in Drosophila females. Nature 579:101-105. doi:10.1038/s41586-020-2055-9

Wang F, Wang K,...Dickson BJ. 2020b in revision.

Watanabe K, Chiu H, Pfeiffer BD, Wong AM, Hoopfer ED, Rubin GM, Anderson DJ. 2017. A circuit node that integrates convergent input from neuromodulatory and social behaviorpromoting neurons to control aggression in Drosophila. Neuron 95:1112-1128.e7. doi:10.1016/j.neuron.2017.08.017

Wohl M, Ishii K, Asahina K. 2020. Layered roles of fruitless isoforms in specification and function of male aggression-promoting neurons in Drosophila. Elife 9:1-31. doi:10.7554/eLife.52702

Woodworth CH. 1971. Attack elicited in rats by electrical stimulation of the lateral hypothalamus. Physiol Behav 6:345-353. doi:10.1016/0031-9384(71)90166-1

Wu F, Deng B, Xiao N, Wang T, Li Y, Wang R, Shi K, Luo DG, Rao Y, Zhou C. 2020. A neuropeptide regulates fighting behavior in Drosophila melanogaster. Elife 9:1-22. doi:10.7554/eLife.54229

Wu M, Nern A, Ryan Williamson W, Morimoto MM, Reiser MB, Card GM, Rubin GM. 2016. Visual projection neurons in the Drosophila lobula link feature detection to distinct behavioral programs. Elife 5:1-43. doi:10.7554/eLife.21022

Yamamoto D. 2007. The neural and genetic substrates of sexual behavior in Drosophila. Adv Genet. 59:39-66. doi:10.1016/S0065-2660(07)59002-4

Yamamoto D, Koganezawa M. 2013. Genes and circuits of courtship behaviour in Drosophila males. Nat Rev Neurosci. 14:681-692. doi:10.1038/nrn3567

Yang CF, Chiang MC, Gray DC, Prabhakaran M, Alvarado M, Juntti SA, Unger EK, Wells JA, Shah NM. 2013. Sexually dimorphic neurons in the ventromedial hypothalamus govern mating in both sexes and aggression in males. Cell 153:896-909. doi:10.1016/j.cell.2013.04.017

Yuan Q, Song Y, Yang CH, Jan LY, Jan YN. 2014. Female contact modulates male aggression via a sexually dimorphic GABAergic circuit in Drosophila. Nat Neurosci 17:81-88. doi:10.1038/nn.3581

Zheng Z, Lauritzen JS, Perlman E, Saalfeld S, Fetter RD, Bock DD. 2018. A complete electron microscopy volume of the brain of adult Drosophila melanogaster. Cell 174:730-743. doi:10.1016/j.cell.2018.06.019

Zhou C, Pan Y, Robinett CC, Meissner GW, Baker BS. 2014. Central brain neurons expressing doublesex regulate female receptivity in Drosophila. Neuron 83:149-163. doi:10.1016/j.neuron.2014.05.038

Zhou C, Rao Yong, Rao Yi. 2008. A subset of octopaminergic neurons are important for 
bioRxiv preprint doi: https://doi.org/10.1101/2020.05.27.118810; this version posted May 27, 2020. The copyright holder for this preprint (which

was not certified by peer review) is the author/funder, who has granted bioRxiv a license to display the preprint in perpetuity. It is made available under aCC-BY 4.0 International license.

961 Zwarts L, Versteven M, Callaerts P. 2012. Genetics and neurobiology of aggression in

962 Drosophila. Fly (Austin) 6:35-48. doi:10.4161/fly.19249 


\section{Supplementary Figures}
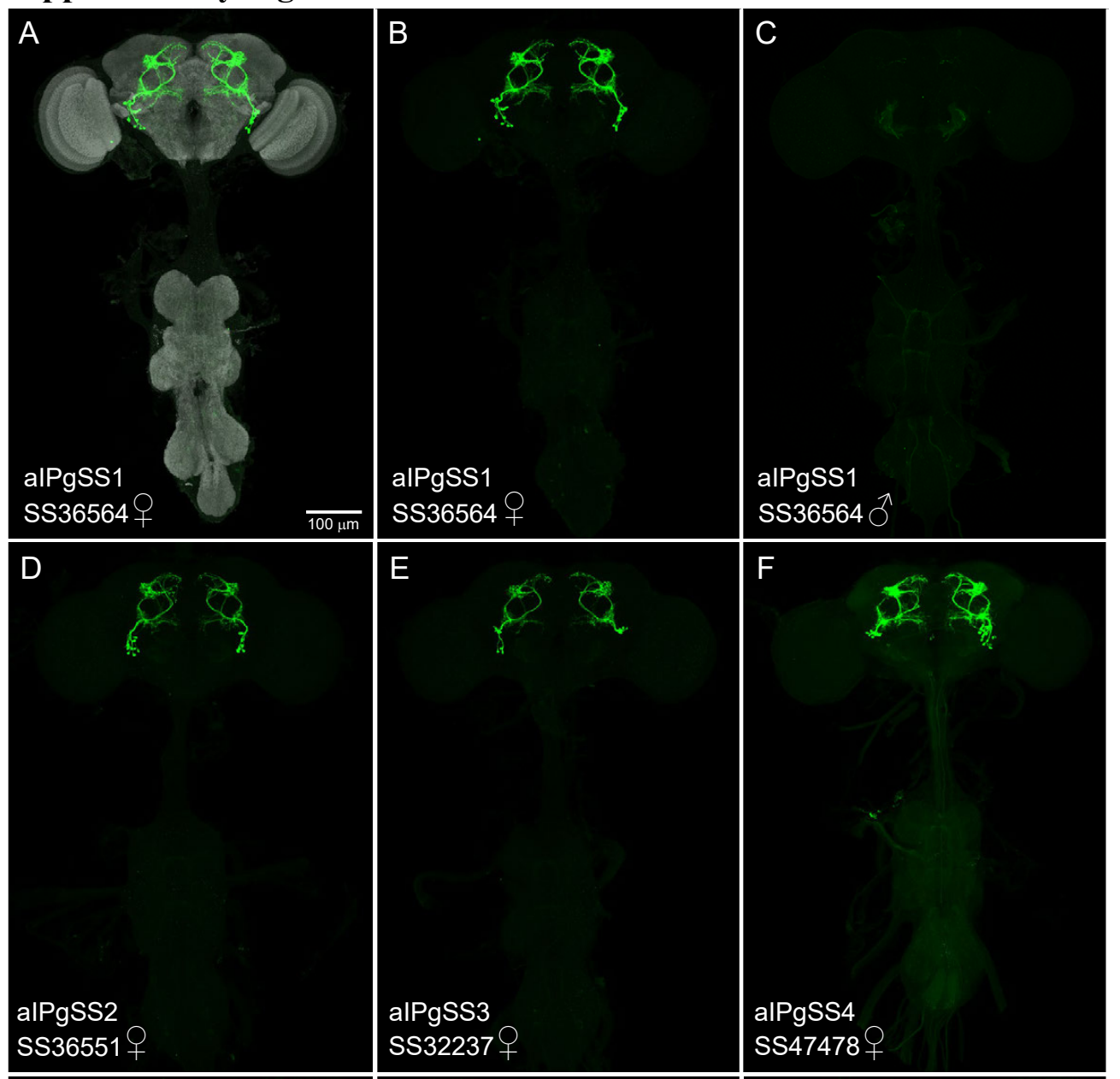

Figure 1 - figure supplement 1. Expression patterns of aIPg split-GAL4 lines. (A - F) Maximum intensity projections $(20 \mathrm{x})$ of the brains and ventral nerve cords of the indicated split-GAL4 lines crossed to 20xUASCsChrimson::mVenus and stained with anti-GFP antibody are shown. As the images are visualizations of the optogenetic effector itself, these images indicate the relative expression levels of the effector in the different splitGAL4 lines. Sex of the imaged brain is indicated as is the stock name of split-GAL4 line. The scale bar shown in A applies to panels A - F. ( $\left.D^{\prime}-F^{\prime}\right)$ Enlargements of the central brain of the images shown in $D-F$. (A - C) The SS36564 (aIPgSS1) expression pattern is shown; the neuropil reference channel is shown in A (gray). SS36564 expresses in $11.3 \pm 1.5$ cells per female brain hemisphere $(n=8)$. Note the lack of expression in aIPg neurons in the male central nervous system (C) imaged under identical conditions to that of the female (B). (D, D') Expression pattern of SS36551 (aIPgSS2) in females. SS365551 expresses in 8.9 \pm 1.4 cells per female brain hemisphere $(\mathrm{n}=8)$. A few cells with distinct, but related morphology are observed in males. (E, E') Expression pattern of SS32237 (aIPgSS3) in females. SS32237 expresses in $7.8 \pm 0.89$ cells per female brain hemisphere $(n=8)$. A few cells with distinct, but related morphology are observed in males. (F, F'). Expression pattern of SS47478 (aIPgSS4) in females; this line expresses in $12.8 \pm 1.8$ cells per female brain hemisphere $(\mathrm{n}=8)$. No expression was seen in males. 


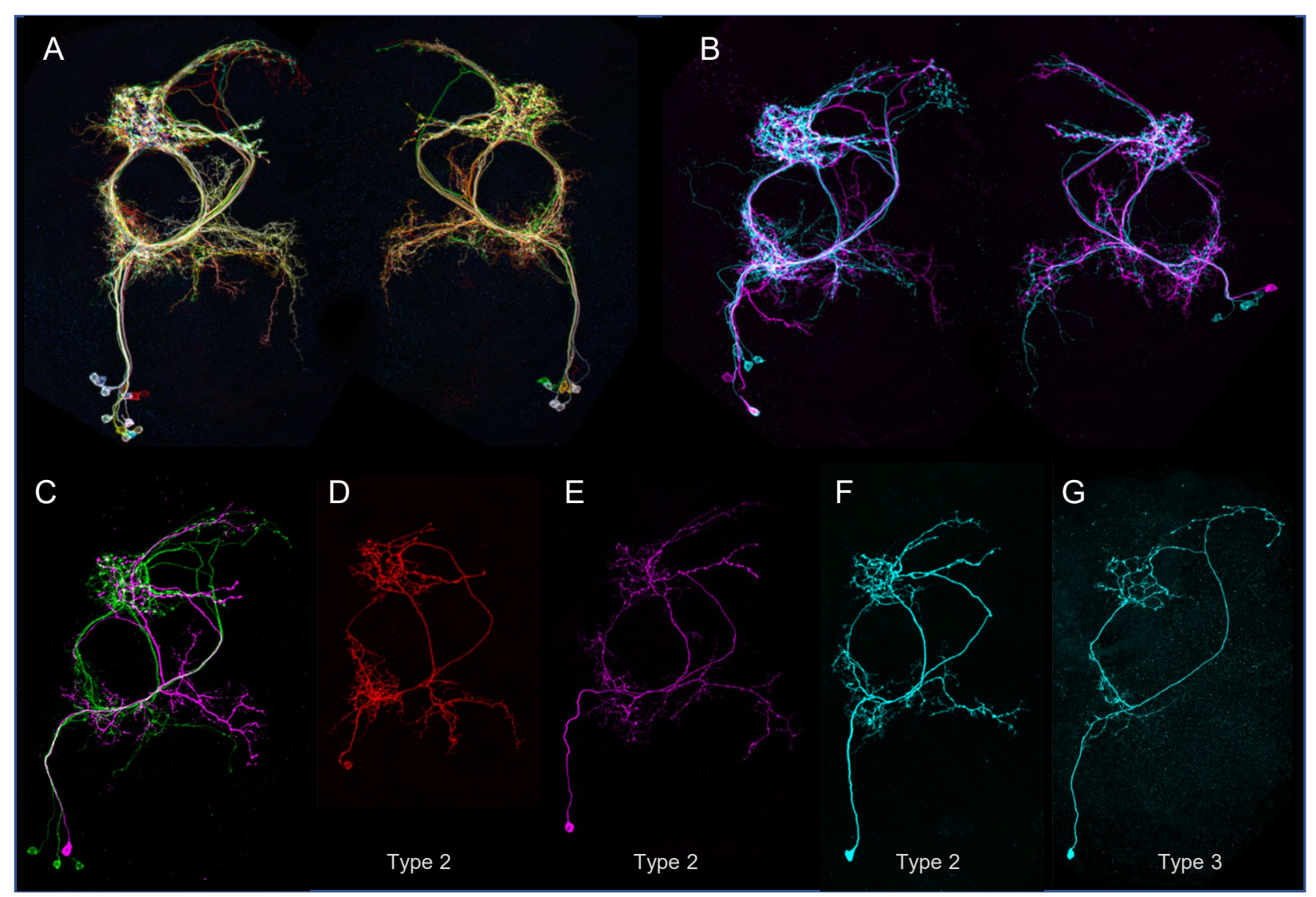

Figure 1 - figure supplement 2. Morphologies of individual aIPg type $1-\mathbf{3}$ neurons. The images in all panels were generated by stochastic labeling of aIPg split-GAL4 lines using the MultiColor FlipOut method (Nern et al. 2015). (A, B) Images from SS36564 (aIPgSS1). Both brain hemispheres, and multiple aIPg cells, are shown. (C, E, $\mathrm{F}, \mathrm{G})$ Images from SS36551 (aIPgSS2). One brain hemisphere is shown. (D) Image from SS47478 (aIPgSS4). One brain hemisphere is shown. (D, E, F) show examples of type 2 aIPg cells, while (G) shows a type 3 aIPg cell. 


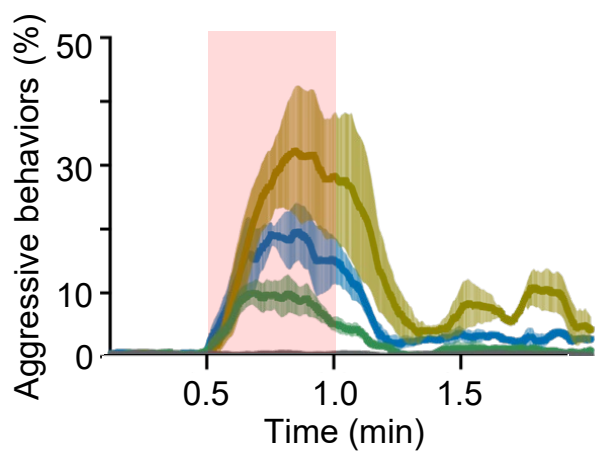

- EmptySS > Chrimson

- alPgSS1 > Chrimson

- alPgSS2 > Chrimson

— alPgSS3 > Chrimson
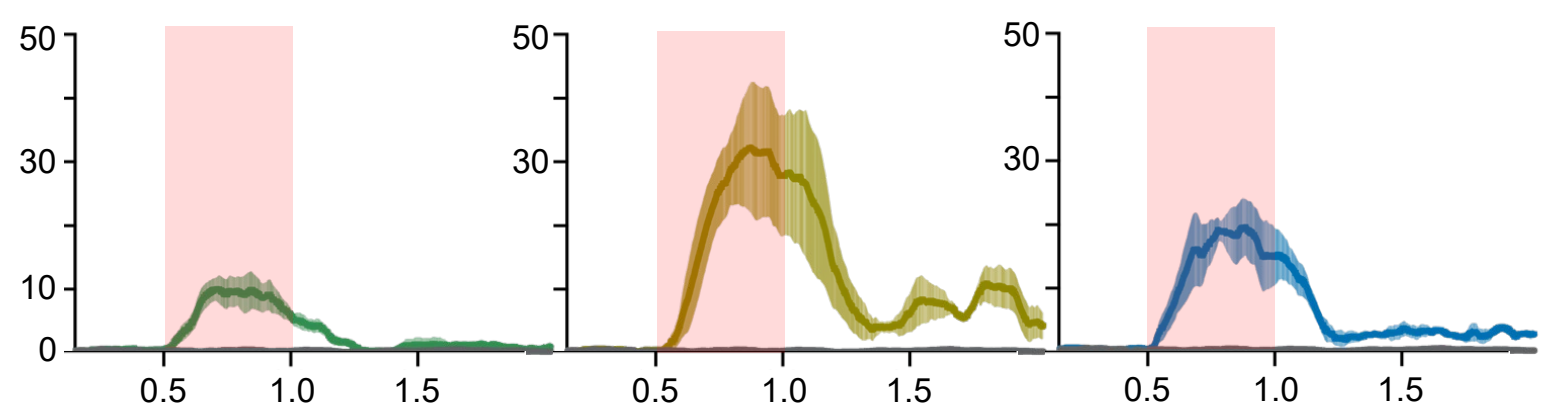

Figure 1 - figure supplement 3. Additional aIPg split-GAL4 lines also induce aggressive behavior. Percentage of flies engaging in aggressive behaviors over the course of a 2 -minute trial during which a 30 -second $0.1 \mathrm{~mW} / \mathrm{mm}^{2}$ continuous light stimulus was delivered is shown for three different split-GAL4 lines that drive expression in the aIPg cells. The expression pattern of these three aIPgSS lines are shown in Figure 1 - figure supplement 1 . The red shading indicates the stimulus period. The mean is represented as a solid line and shaded error bars represent variation between experiments. For clarity, the individual plots for each line and the EmptySS $>$ Chrimson are also shown. Each experiment included approximately 15 flies. EmptySS $>$ Chrimson, $n=7$ experiments; aIPgSS1 > Chrimson, $\mathrm{n}=5$ experiments; aIPgSS $2>$ Chrimson, $\mathrm{n}=3$ experiments; aIPgSS $3>$ Chrimson, $\mathrm{n}=4$ experiments. 


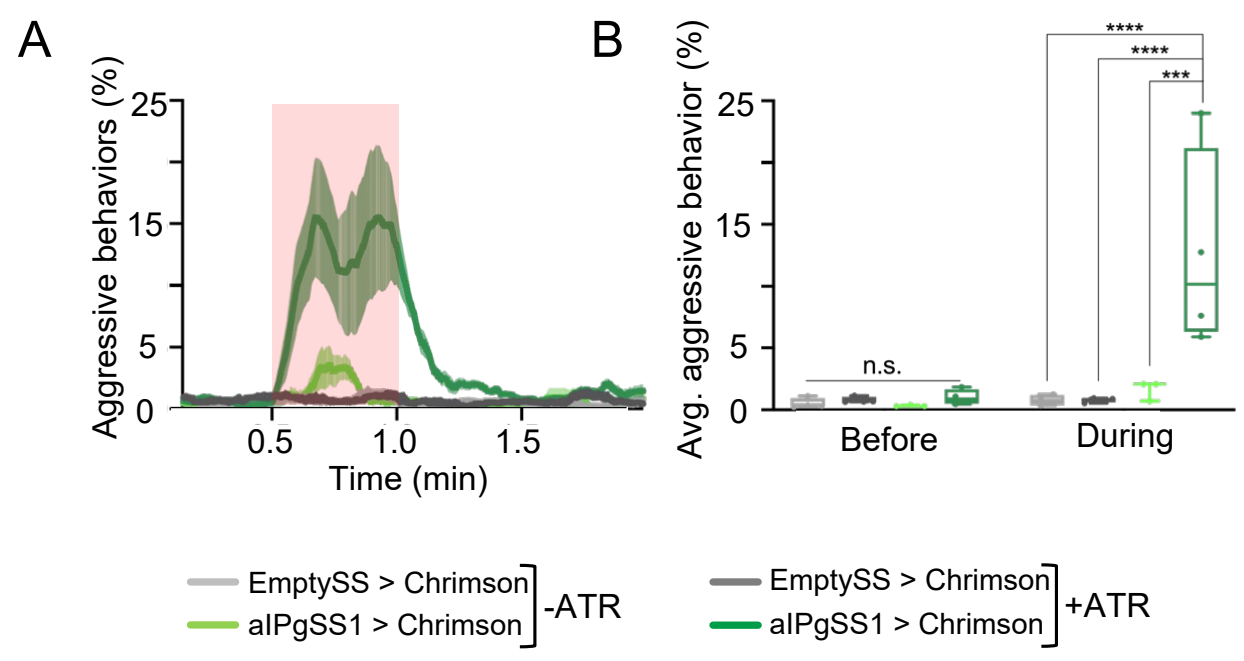

Figure 1 - figure supplement 4. Optogenetic activation of aggression with Chrimson requires on feeding all trans-retinal. (A) Percentage of flies that engaged in aggressive behaviors. Flies were raised with food supplemented with all trans-retinal (+ATR) or not (-ATR). A 30 -second $0.1 \mathrm{~mW} / \mathrm{mm}^{2}$ continuous light stimulus was delivered $30 \mathrm{sec}$ after the start of a 2-minute trial as indicated by the red shading. The mean is represented as a solid line and shaded error bars represent variation between experiments. (B) Average percentage of flies engaging in aggressive behaviors over the 30 second period prior to or during the stimulus delivery in A. Points represent separate experiments consisting of approximately 15 flies. +ATR: EmptySS $>$ Chrimson, $n=4$ experiments; aIPgSS1 > Chrimson, $\mathrm{n}=4$ experiments; -ATR: EmptySS $>$ Chrimson, $\mathrm{n}=4$ experiments; aIPgSS $1>$ Chrimson, $\mathrm{n}$ $=3$ experiments. Box-and-whisker plots show median and interquartile range (IQR); whiskers show range. A twoway ANOVA with a Tukey's multiple comparisons post-hoc test was used for statistical analysis. Asterisk indicates significance from $0:{ }^{* *} \mathrm{p}<0.001 ; \mathrm{n} . \mathrm{s}$, not significant. 

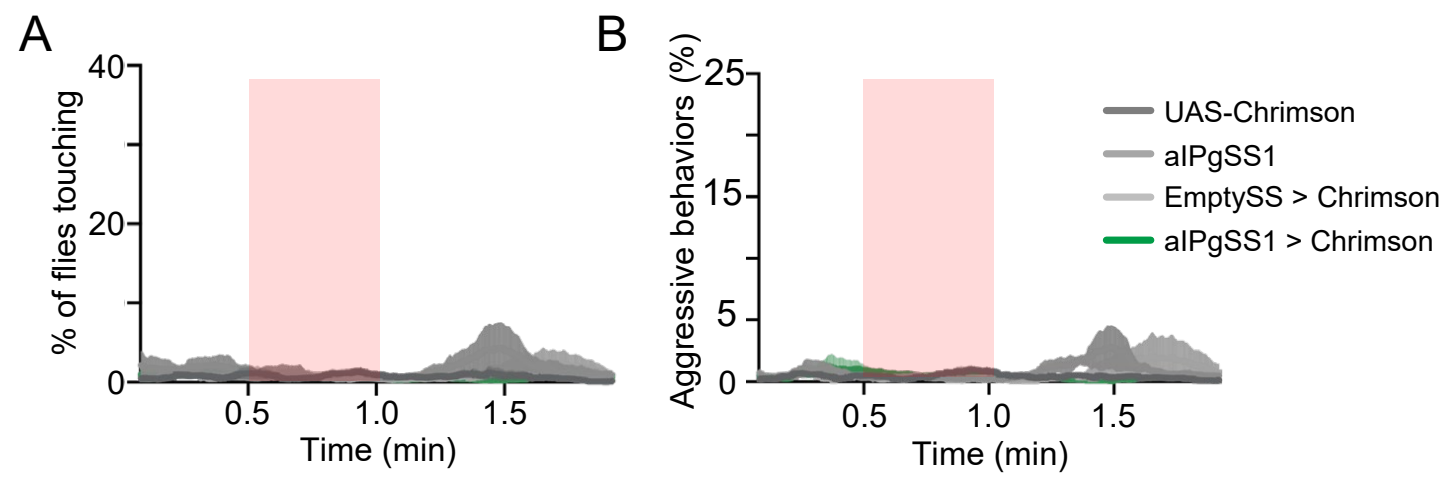

Figure 1 - figure supplement 5. Optogenetic stimulation of aIPgSS1>Chrimson males does not result in aggressive behavior. (A - B) Percentage of male flies touching (A) or performing aggressive behaviors (fencing and head butting) (B) over the course of a 2-minute trial during which a 30 -second $0.4 \mathrm{~mW} / \mathrm{mm}^{2}$ continuous light stimulus was delivered as indicated by the red bar. The mean is represented as a solid line and shaded error bars represent variation between experiments. Each experiment included approximately 15 flies. 20xUAS-CsChrimson, $\mathrm{n}$ $=5$ experiments; aIPgSS1, $\mathrm{n}=4$ experiments; EmptySS > 20xUAS-CsChrimson, $\mathrm{n}=3$ experiments; aIPgSS $1>$ 20xUAS-CsChrimson, $\mathrm{n}=6$ experiments. 
bioRxiv preprint doi: https://doi.org/10.1101/2020.05.27.118810; this version posted May 27, 2020. The copyright holder for this preprint (which was not certified by peer review) is the author/funder, who has granted bioRxiv a license to display the preprint in perpetuity. It is made available under aCC-BY 4.0 International license.

\begin{tabular}{|c|c|c|c|c|c|c|c|}
\hline gene ID & $\begin{array}{c}\text { gene } \\
\text { symbol }\end{array}$ & alPgSS3 & alPgSS4 & $\begin{array}{c}\text { pC1dSS1 } \\
\text { (pC1d only) }\end{array}$ & $\begin{array}{c}\text { pC1dSS3 } \\
\text { (pC1d \& e) }\end{array}$ & $\begin{array}{c}\text { pC1eSS1 } \\
\text { (pC1e only) }\end{array}$ & $\begin{array}{c}\text { pC1eSS3 } \\
\text { (pC1e only) }\end{array}$ \\
\hline \hline FBgn0032840 & sNPF & $457 \pm 194$ & $62.5 \pm 28$ & $97 \pm 98$ & $45 \pm 79$ & $2 \pm 3$ & $0 \pm 0$ \\
FBgn0036934 & $s N P F-R$ & $73.9 \pm 55.9$ & $44.2 \pm 22.6$ & $3 \pm 4$ & $70 \pm 73$ & $53 \pm 35$ & $92 \pm 54$ \\
FBgn0000303 & ChAT & $86.7 \pm 20$ & $68.2 \pm 37$ & $5245 \pm 573$ & $3049 \pm 274$ & $4989 \pm 100$ & $4192 \pm 147$ \\
FBgn0004516 & Gad1 & $0 \pm 0$ & $0 \pm 0$ & $28 \pm 25$ & $59 \pm 79$ & $0 \pm 0$ & $32 \pm 45$ \\
FBgn0260964 & Vmat & $0 \pm 0$ & $0 \pm 0$ & $37 \pm 52$ & $72 \pm 117$ & $0 \pm 0$ & $0 \pm 0$ \\
FBgn0004652 & fru & $205 \pm 94$ & $632 \pm 212$ & $121 \pm 40$ & $84 \pm 50$ & $192 \pm 47$ & $142 \pm 10$ \\
FBgn0000504 & $d s x$ & $0 \pm 0$ & $0 \pm 0$ & $665 \pm 142$ & $306 \pm 144$ & $376 \pm 48$ & $345 \pm 51$ \\
\hline
\end{tabular}

Figure 1 - figure supplement 6. aIPg neurons are cholinergic, fru ${ }^{+}$and $\mathrm{sNPF}^{+} ; \mathbf{p C 1}$ neurons are cholinergic and $\mathbf{d s x}^{+}$. Expression (TPM) of genes related to neurotransmitters and sex determination pathways, $s N P F, s N P F-R$, $d s x$ and fru. Cells were dissociated from dissected brains and sorted on the basis of their expression of the indicated split-GAL4 driver: $\operatorname{aIPgSS} 3(\mathrm{n}=2), \operatorname{aIPgSS} 4(\mathrm{n}=3), \operatorname{pCldSS} 1(\mathrm{n}=3), \operatorname{pCldSS} 3(\mathrm{n}=6), \operatorname{pCleSS} 1(\mathrm{n}=3)$, and pCleSS3 $(n=3)$. Cell sorting and RNA profiling was done as described in Aso et al. (2019). The mean and standard deviation of the indicated number of biological repeats are shown. Complete transcript data were deposited to NCBI Gene Expression Omnibus [author note: submission will be done while paper is under review]. 

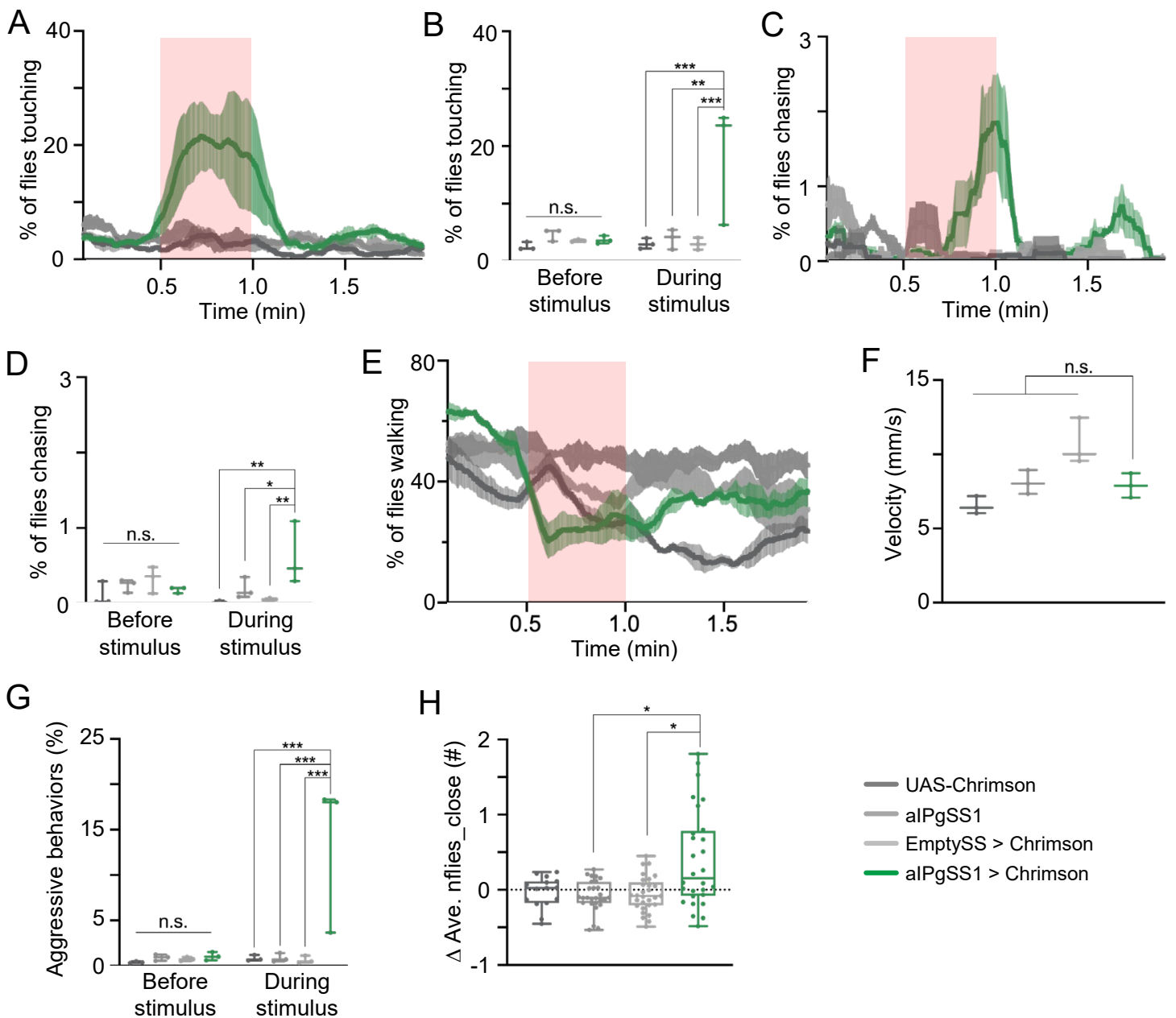

Figure 1 - figure supplement 7 . Changes in behavioral metrics in females following activation of aIPgSS1 neurons. (A, C, E) Percentage of female flies touching (A), chasing (C), or walking (E) over the course of a 2minute trial during which a 30 -second $0.4 \mathrm{~mW} / \mathrm{mm}^{2}$ continuous light stimulus was delivered. (B, D) Average percentage of flies touching (B) or chasing (D) over the 30-second period prior to or during the stimulus delivery in A or C, respectively. (F) Velocity over the course of a 2-minute trial including the stimulus period. (G) Average percentage of flies engaging in aggressive behaviors over the 30 -second period prior to or during the stimulus delivery in Figure 1D. (A - G) 20xUAS-CsChrimson, $\mathrm{n}=3$ experiments; aIPgSS1, $\mathrm{n}=3$ experiments; EmptySS > 20xUAS-CsChrimson, $\mathrm{n}=3$ experiments; aIPgSS1 > 20xUAS-CsChrimson, $\mathrm{n}=3$ experiments. (H) Difference in the average number of flies within two body lengths between the 30 -seconds prior to and during the stimulus. 20xUAS-CsChrimson, $\mathrm{n}=15$ flies; aIPgSS1, $\mathrm{n}=27$ flies; EmptySS $>$ 20xUAS-CsChrimson, $\mathrm{n}=29$ flies; aIPgSS1 $>20 x U A S-C s C h r i m s o n, ~ n=28$ flies. Points represent separate experiments consisting of approximately 15 flies (B, $\mathrm{D}, \mathrm{E}, \mathrm{G})$ or an individual fly $(\mathrm{H})$. Analyses were performed on the same data set used in Figure 1. Box-and-whisker plots show median and interquartile range (IQR); whiskers show range. A two-way ANOVA with a Tukey's multiple comparisons post-hoc test was used for statistical analysis. Asterisk indicates significance from $0:{ }^{*} \mathrm{p}<0.05$; $* * \mathrm{p}<0.01 ; * * * \mathrm{p}<0.001 ; \mathrm{n} . \mathrm{s}$, not significant. 

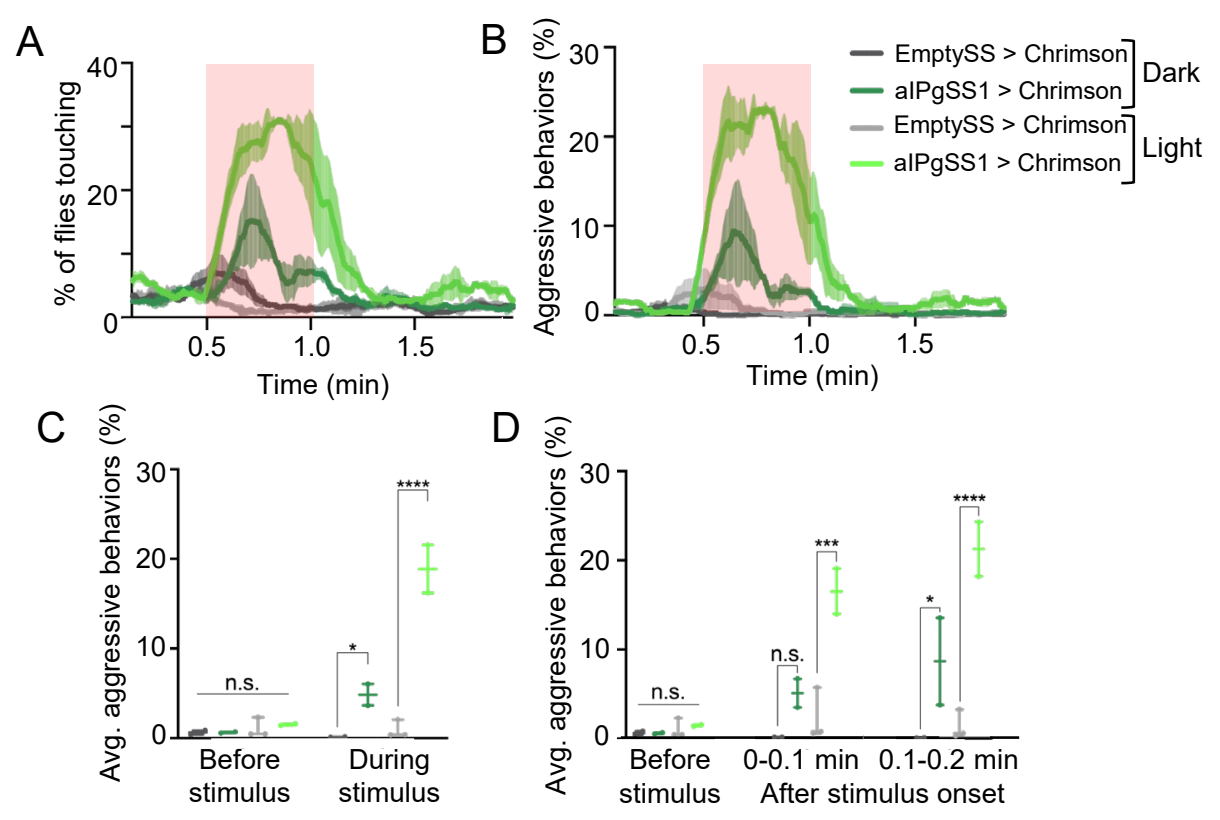

Figure 1 - figure supplement 8. Comparison of the results of aIPgSS1 stimulation in light and dark

conditions. $(\mathrm{A}-\mathrm{B})$ Percentage of female flies touching $(\mathrm{A})$ or engaging in aggressive behaviors (B) over the course of a 2-minute trial during which a 30 -second $0.1 \mathrm{~mW} / \mathrm{mm}^{2}$ continuous light stimulus was delivered. (C) Average percentage of flies engaging in aggressive behaviors over the 30 -second period prior to or during the stimulus delivery in B. (D) Average percentage of flies engaging in aggressive behaviors over the 30-second period prior to, the first 6 seconds, or the second 6 seconds during the stimulus delivery in B. Points in C and D represent separate experiments consisting of approximately 15 flies. Light: EmptySS $>$ Chrimson, $n=3$ experiments; aIPgSS1 > Chrimson, $\mathrm{n}=2$ experiments; Dark: EmptySS $>$ Chrimson, $\mathrm{n}=2$ experiments; aIPgSS1 $>$ Chrimson, $\mathrm{n}=2$ experiments. Box-and-whisker plots show median and interquartile range (IQR); whiskers show range. A two-way ANOVA with a Tukey's multiple comparisons post-hoc test was used for statistical analysis. Asterisk indicates significance from 0 : $* \mathrm{p}<0.05 ; * * * \mathrm{p}<0.001 ; * * * * \mathrm{p}<0.0001$; n.s., not significant. 

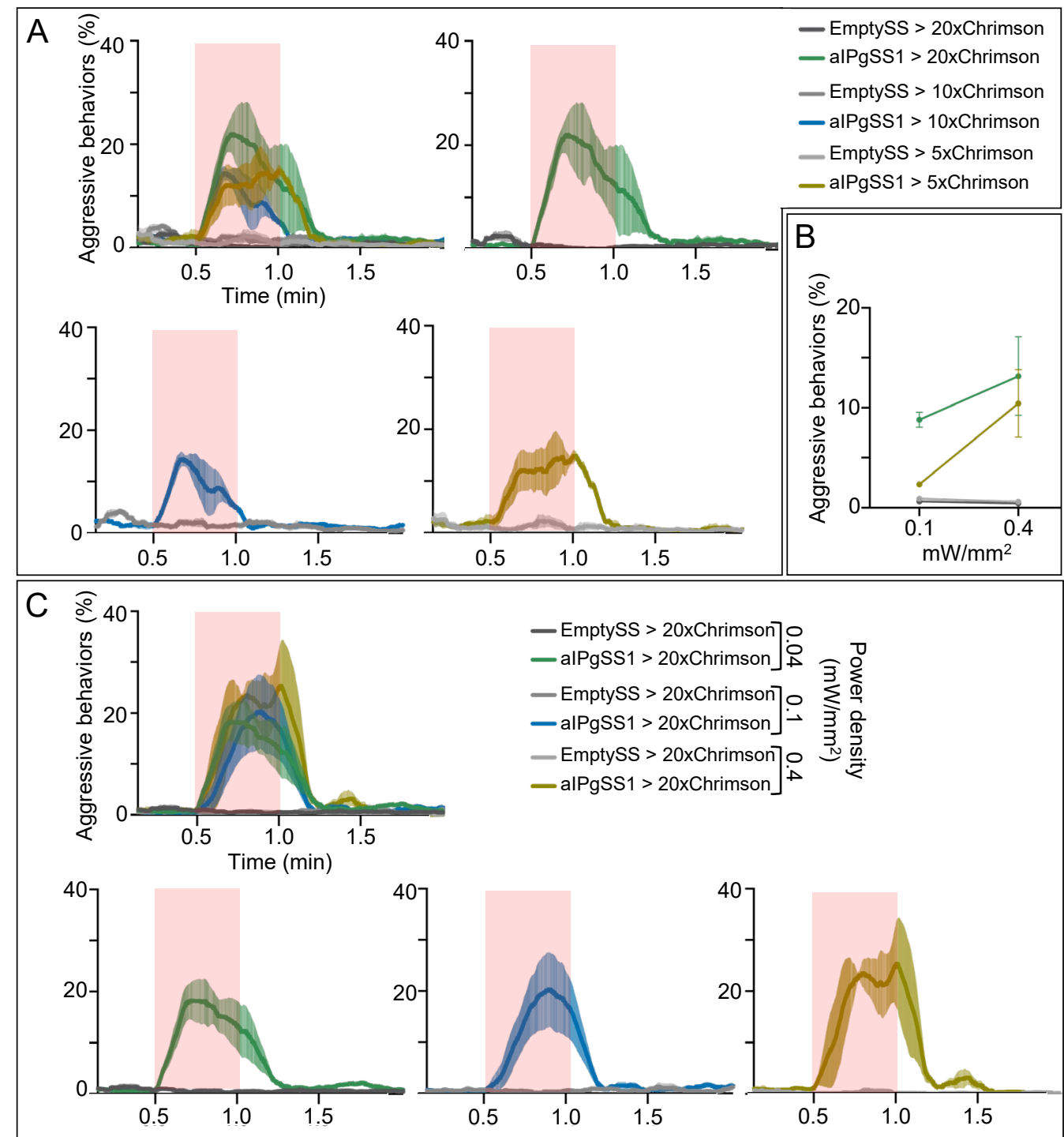

Figure 1 - figure supplement 9. Behavioral effects of effector strength and stimulus delivery. (A) Percentage of flies that carry 5x, 10x, or 20x UAS-Chrimson effectors that engage in aggressive behaviors over the course of a 2minute trial during which a 30 -second $0.1 \mathrm{~mW} / \mathrm{mm}^{2}$ continuous light stimulus was delivered as indicated by the red shading. Individual curves are shown for comparison and each experiment included approximately 15 flies. EmptySS > 20xUAS-Chrimson, $\mathrm{n}=2$ experiments; aIPgSS1 > 20xUAS-Chrimson, $\mathrm{n}=3$ experiments; EmptySS > 10xUAS-Chrimson, $\mathrm{n}=3$ experiments; aIPgSS1 $>10 x \mathrm{xAS}$-Chrimson, $\mathrm{n}=4$ experiments; EmptySS $>5 \mathrm{xUAS}$ Chrimson, $\mathrm{n}=3$ experiments; aIPgSS $1>5 \mathrm{xUAS}$-Chrimson, $\mathrm{n}=3$ experiments. (B) Average percentage of flies with either the $5 \mathrm{x}$ or $20 \mathrm{x}$ UAS-Chrimson effector that perform aggressive behaviors over the 30 -second period during the stimulus delivery. Each experiment included approximately 15 flies. $0.1 \mathrm{~mW} / \mathrm{mm}^{2}$ : EmptySS $>5 x$ XAS-Chrimson (light gray), $\mathrm{n}=6$ experiments; aIPgSS1 $>5 x \mathrm{x}$ AS-Chrimson (gold), $\mathrm{n}=5$ experiments; EmptySS $>20 \mathrm{xUAS}$ Chrimson (dark gray), $\mathrm{n}=6$ experiments; aIPgSS1 $>20 x \mathrm{xAS}$-Chrimson (green), $\mathrm{n}=8$ experiments; $0.4 \mathrm{~mW} / \mathrm{mm}^{2}$ : EmptySS $>5 x$ UAS-Chrimson, $\mathrm{n}=6$ experiments; aIPgSS1 $>5 x$ UAS-Chrimson, $\mathrm{n}=9$ experiments; EmptySS $>$ 20xUAS-Chrimson, $\mathrm{n}=3$ experiments; aIPgSS1 $>$ 20xUAS-Chrimson, $\mathrm{n}=5$ experiments. Bars are mean $+/$ - S.E.M. (C) Percentage of flies engaging in aggressive behaviors over the course of a 2-minute trial during which a 30second $0.4,0.1$, or $0.04 \mathrm{~mW} / \mathrm{mm}^{2}$ continuous light stimulus was delivered. Individual curves are shown for comparison and each experiment included approximately 15 flies. $0.04 \mathrm{~mW} / \mathrm{mm}^{2}$ : EmptySS $>$ Chrimson, $\mathrm{n}=5$ experiments; aIPgSS $1>$ Chrimson, $\mathrm{n}=6$ experiments; $0.1 \mathrm{~mW} / \mathrm{mm}^{2}$ : EmptySS $>$ Chrimson, $\mathrm{n}=4$ experiments; aIPgSS1 $>$ Chrimson, $\mathrm{n}=4$ experiments; $0.4 \mathrm{~mW} / \mathrm{mm}^{2}$ : EmptySS $>$ Chrimson, $\mathrm{n}=3$ experiments; aIPgSS1 $>$ Chrimson, $\mathrm{n}=3$ experiments. 

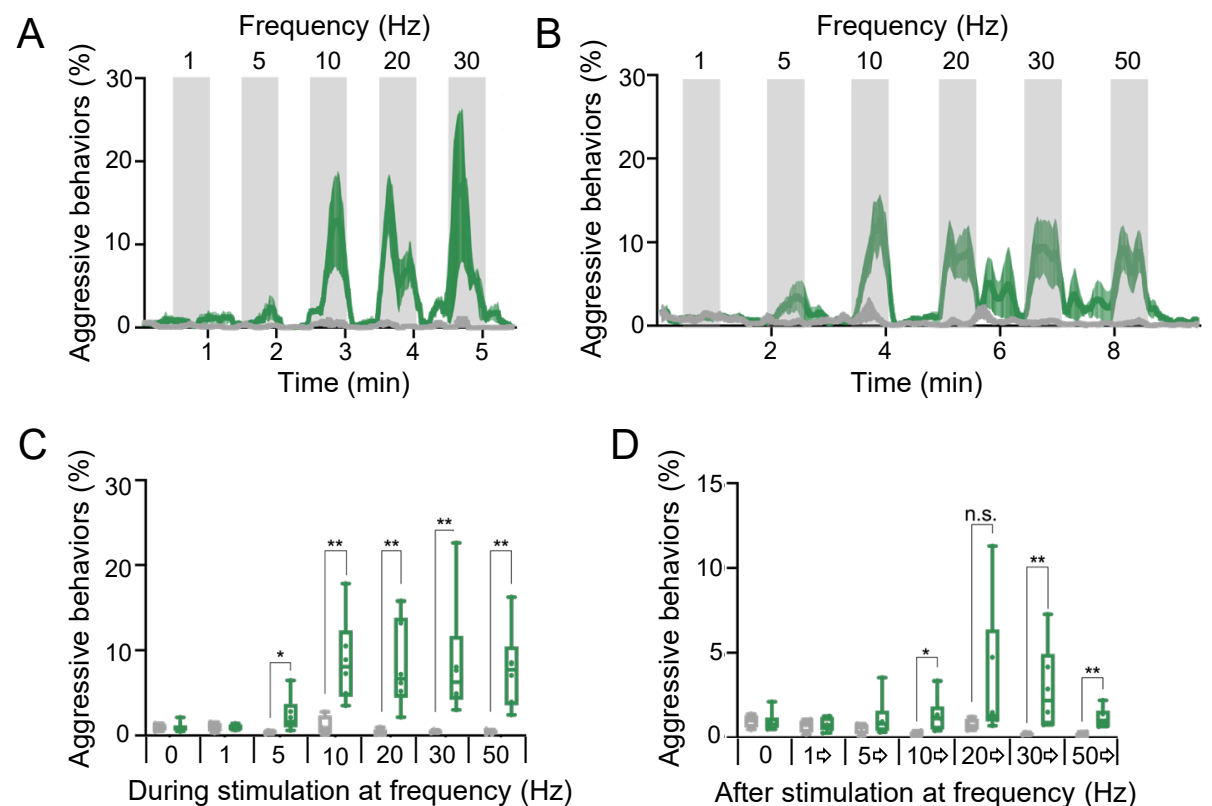

Figure 1 - figure supplement 10. Higher frequency optogenetic stimulation increases the persistence of aggressive behaviors. (A - B) Blocks of 30-second photostimulation (grey bars) with increasing stimulation frequency separated by 30 -second (A) or 60-second (B) intervals were delivered sequentially to females. Light was delivered at $0.1 \mathrm{~mW} / \mathrm{mm}^{2}$ with at a $10-\mathrm{ms}$ pulse width, but with increasing pulse frequency. Numbers above the grey bars correspond to the frequency over the 30 -second stimulus period. The pulse period and pulse number during each period was as follows: $1000 \mathrm{~ms}, 30 ; 200 \mathrm{~ms}, 150 ; 100 \mathrm{~ms}, 300 ; 50 \mathrm{~ms}, 600 ; 33 \mathrm{~ms}, 909 ; 20 \mathrm{~ms}, 1500$. aIPgSS1 > 20xUAS-Chrimson is shown in green; EmptySS > 20xUAS-Chrimson is shown in gray. The mean is represented as a solid line and error bars represent variation between experiments. Each experiment included approximately 15 flies. A: EmptySS > 20xUAS-Chrimson, $\mathrm{n}=3$ experiments; aIPgSS1 > 20xUAS-Chrimson, $\mathrm{n}=3$ experiments; $\mathrm{B}$ : EmptySS $>20 x U A S-C h r i m s o n, ~ n=4$ experiments; aIPgSS1 $>20 x$ UAS-Chrimson, $n=5$ experiments. (C) Average percentage of flies performing aggressive behaviors over the 30 -second period during stimulus delivery in B. (D) Average percentage of flies performing aggressive behaviors over the 30 -second period before $1 \mathrm{~Hz}$, the 60 -second periods between subsequent stimuli, and the 30 -seconds after $50 \mathrm{~Hz}$ stimulation in B. Points indicate separate experiments. Box-and-whisker plots show median and interquartile range (IQR); whiskers show range. MannWhitney $U$-tests were used for statistical analysis. Asterisk indicates significance from $0:{ }^{*} p<0.05 ; * * p<0.01$; n.s., not significant. 
A

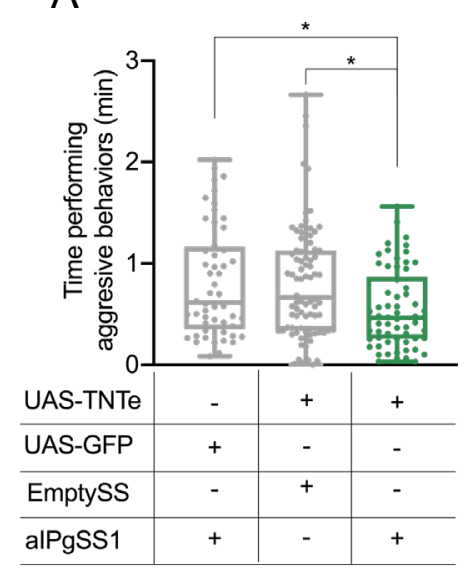

C

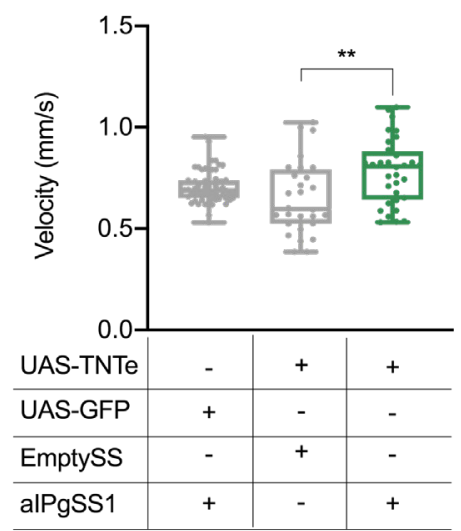

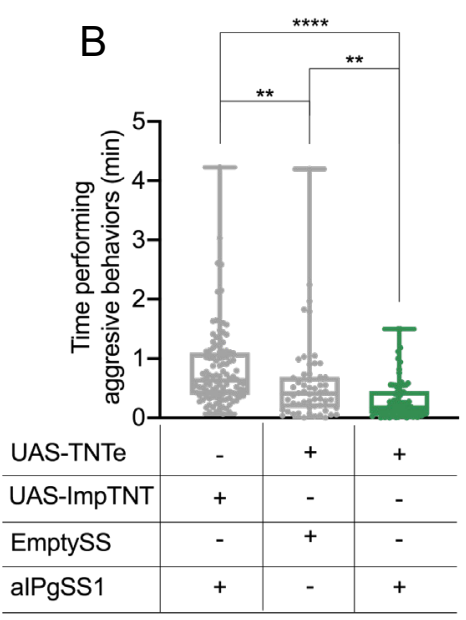

$\mathrm{D}$

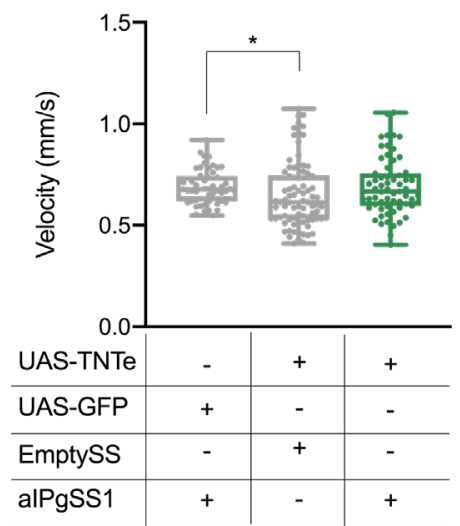

Figure 2 - figure supplement 1 . aIPg inactivation reproducibly decreases aggressive behaviors but not velocity. (A - B) Total time an individual spent performing aggressive behaviors over a 30-minute trial. Plots in A and $\mathrm{B}$ each represent the pooled data from 4 and 5 testing days, respectively, of separate biological repeats. The experiments in A and B differ in that a different negative control UAS line was used: UAS-GFP in A and UASImpTNT in B; ImpTNT is an inactive form of TNT (see Methods). Experiments were scored using an automated classifier generated with JAABA (see Methods). Points indicate individual flies. A: aIPgSS1 $>$ UAS-GFP, $n=52$ flies; EmptySS > UAS-TNTe, $\mathrm{n}=82$ flies; aIPgSS1 > UAS-TNTe, $\mathrm{n}=62$ flies; B: aIPgSS1 > UAS-ImpTNTe, $\mathrm{n}=$ 110 flies; EmptySS $>$ UAS-TNTe, $n=56$ flies; aIPgSS1 $>$ UAS-TNTe, $n=74$ flies. (C) Average velocity over the 30-minute trial shown in Figure 2. Points indicate individual flies. aIPgSS1 > UAS-GFP, $\mathrm{n}=54$ flies; EmptySS > UAS-TNTe, $\mathrm{n}=28$ flies; aIPgSS1 > UAS-TNTe, $\mathrm{n}=30$ flies. (D) Average velocity over the 30 -minute trial in A. Box-and-whisker plots show median and interquartile range (IQR); whiskers show either $1.5 \times \mathrm{IQR}$ of the lower and upper quartiles. Kruskal-Wallis and Dunn's post hoc tests were used for statistical analysis. Asterisk indicates significance from $0: * \mathrm{p}<0.05 ; * * \mathrm{p}<0.01 ; * * * * \mathrm{p}<0.0001$. 
A

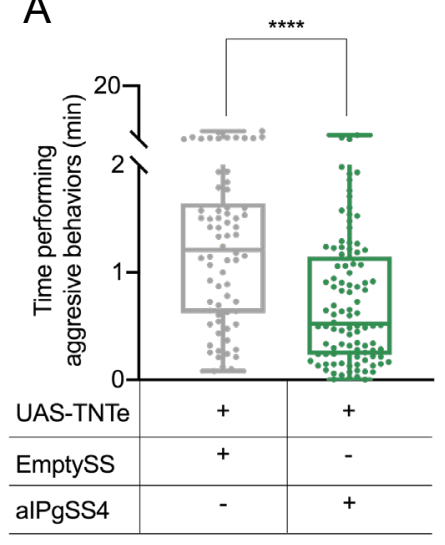

C

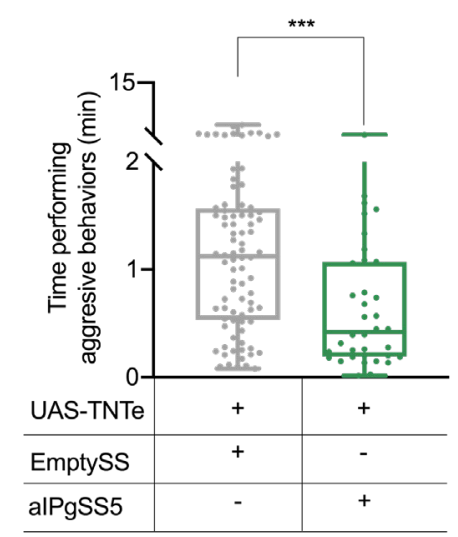

$\mathrm{B}$
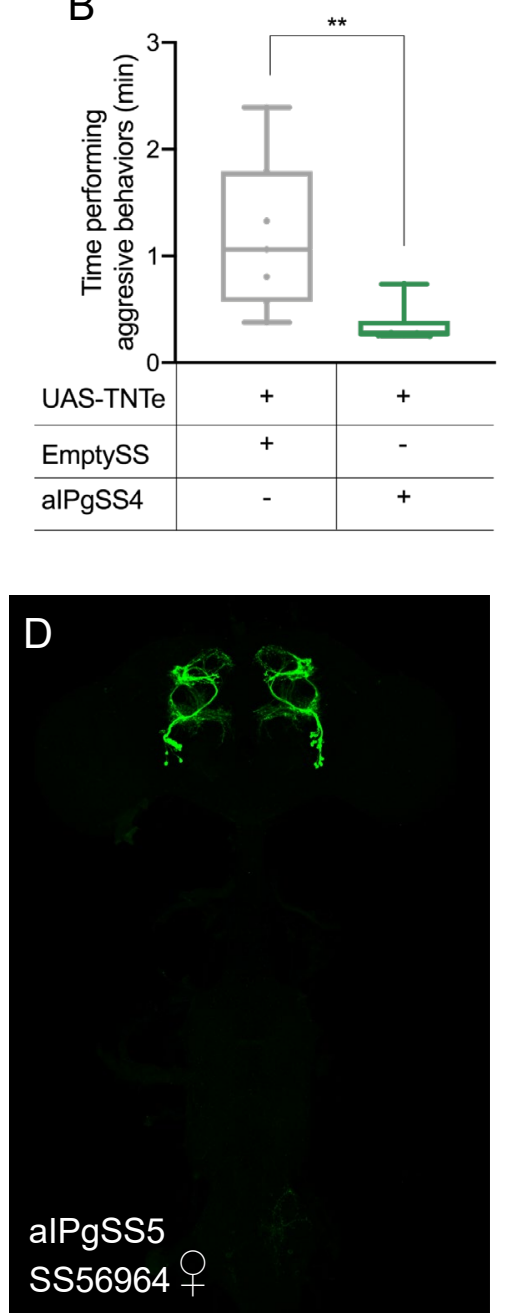

Figure 2 - figure supplement 2. Inactivation of aIPg neurons using additional split-GAL4 lines also decreases aggressive behaviors. $(\mathrm{A}-\mathrm{C}$ ) Total time an individual spent performing aggressive behaviors over a 30-minute trial. Experiments in A - C were pooled from 3, 2 and 2 testing days, respectively. Experiments in B were manually scored with JWatcher software, whereas the experiments in A and C were scored using an automated classifier generated with JAABA (see methods). Points indicate individual flies. A: EmptySS $>$ UAS-TNTe, $\mathrm{n}=70$ flies; aIPgSS4 > UAS-TNTe, $\mathrm{n}=102$ flies; B: EmptySS > UAS-TNTe, $\mathrm{n}=7$ pairs; aIPgSS4 > UAS-TNTe, $\mathrm{n}=6$ pairs; $\mathrm{C}$ : EmptySS > UAS-TNTe, $\mathrm{n}=84$ flies; aIPgSS5 > UAS-TNTe, $\mathrm{n}=36$ flies. (D) Expression pattern of SS56964 (aIPgSS5); this line expresses in 15.8 \pm 0.4 cells per female brain hemisphere $(n=6)$. Box-and-whisker plots show median and interquartile range (IQR); whiskers show either $1.5 \times$ IQR of the lower and upper quartiles. KruskalWallis and Dunn's post hoc tests were used for statistical analysis. Asterisk indicates significance from 0 : $* * * \mathrm{p}<0.001 ; * * * * \mathrm{p}<0.0001$. 

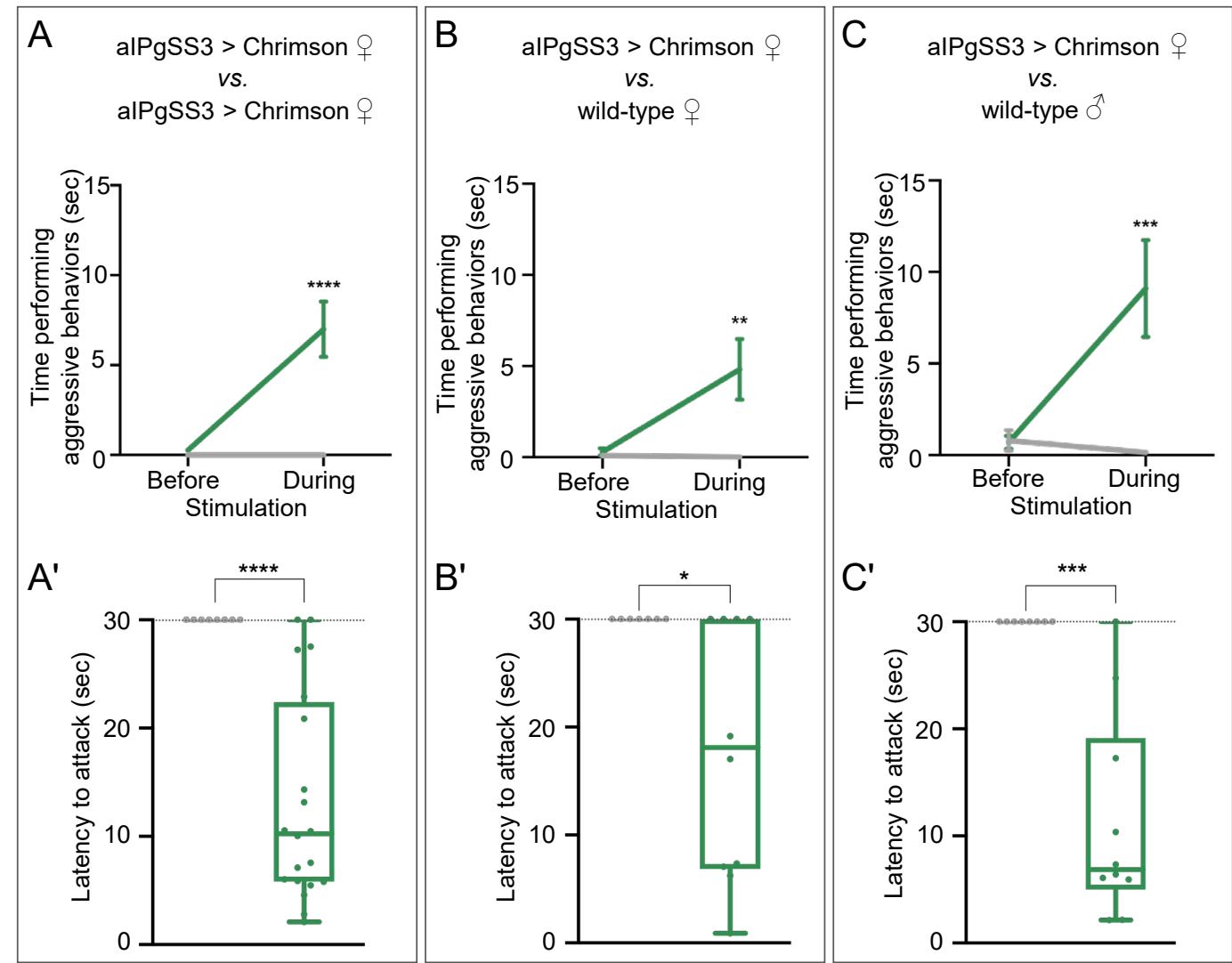

Figure 3 - figure supplement 1 . Increased aggression against wild-type females and males reproduced with a second aIPgSS line. (A - C) Total time an individual spent performing aggressive behaviors in a $16 \mathrm{~mm}$ arena over the 30 second period prior to or during stimulation. The plots refer only to the behavior of aIPgSS $3>$ Chrimson females and each arena contained only two flies: (A) Two aIPgSS3 > Chrimson females; (B) an aIPgSS3 > Chrimson female and a wild-type (Canton-S) female; and (C) a aIPgSS3 > Chrimson female and a wild-type (Canton-S) male. The green line shows the stated genotype; the gray line shows the results when EmptySS > Chrimson was used instead of aIPgSS3 $>$ Chrimson. $\left(\mathrm{A}^{\prime}-\mathrm{C}^{\prime}\right)$ Amount of time during a 30 second $0.1 \mathrm{~mW} / \mathrm{mm}^{2}$ continuous stimulation period until first aggressive encounter. Points indicate individual flies. Dotted lines indicate the end of the trial and error bars are mean +/- S.E.M. Box-and-whisker plots show median and interquartile range (IQR); whiskers show either 1.5 $\times$ IQR of the lower and upper quartiles. A': EmptySS $>20 x U A S-C h r i m s o n, ~ n=16$ flies; aIPgSS3 > 20xUAS-Chrimson, $\mathrm{n}=18$ flies; B': EmptySS > 20xUAS-Chrimson, $\mathrm{n}=5$ flies; aIPgSS3 > 20xUAS-Chrimson, $\mathrm{n}=7$ flies; $\mathrm{C}^{\prime}$ : EmptySS $>$ 20xUAS-Chrimson, $\mathrm{n}=8$ flies; aIPgSS3 $>$ 20xUAS-Chrimson, $\mathrm{n}=$ 10 flies. A Mann-Whitney $U\left(\mathrm{~A}^{\prime}-\mathrm{C}^{\prime}\right)$ post hoc test or a two-way ANOVA with a Sidak's multiple comparisons post-hoc test $(A-C)$ was used for statistical analysis. Asterisk indicates significance from $0:{ }^{*} p<0.05 ;{ }^{*} \mathrm{p}<0.01$; $* * * * \mathrm{p}<0.0001 ;$ n.s., not significant. 
bioRxiv preprint doi: https://doi.org/10.1101/2020.05.27.118810; this version posted May 27, 2020. The copyright holder for this preprint (which

was not certified by peer review) is the author/funder, who has granted bioRxiv a license to display the preprint in perpetuity. It is made available under aCC-BY 4.0 International license.

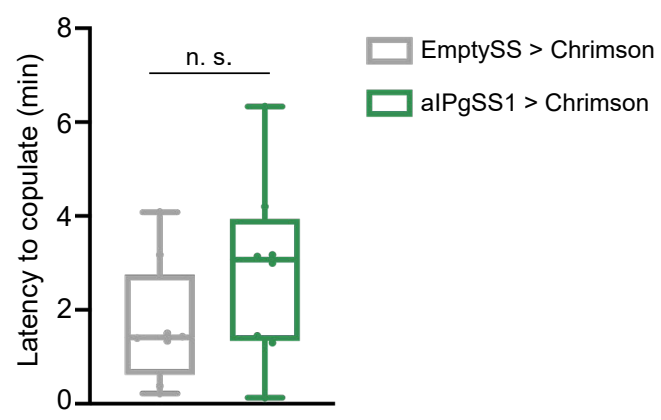

Figure 3 - figure supplement 2. aIPg activation does not significantly alter copulation latency. Amount of time until first copulation event following a $0.4 \mathrm{~mW} / \mathrm{mm}^{2}$ continuous stimulus. Virgin females were added into a $10 \mathrm{~mm}$ arena containing virgin wild type (Canton-S) males immediately prior to the stimulus onset with each stimulus lasting 10 minutes. Points indicate individual flies. EmptySS $>20 x U A S-C h r i m s o n, ~ n=8$ flies; aIPgSS1 $>20 x U A S-$ Chrimson, $\mathrm{n}=8$ flies. Box-and-whisker plots show median and interquartile range (IQR); whiskers show either 1.5 $\times$ IQR of the lower and upper quartiles. A Mann-Whitney $U$ post hoc test was used for statistical analysis. n.s., not significant. 
bioRxiv preprint doi: https://doi.org/10.1101/2020.05.27.118810; this version posted May 27, 2020. The copyright holder for this preprint (which was not certified by peer review) is the author/funder, who has granted bioRxiv a license to display the preprint in perpetuity. It is made available under aCC-BY 4.0 International license.

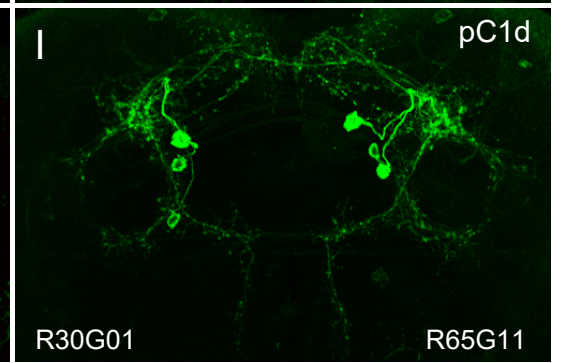

Figure 4 - figure supplement 1. (A - I) Each panel shows the intersection of the indicated enhancers; the enhancer in the lower left was used to drive the split-GAL4 AD domain and the enhancer in the lower right drove the DBD domain. We observed either the $\mathrm{pC} 1 \mathrm{~d}$ or aIPg cell type in each intersection. Nearly all of the pC1d containing intersections also show expression in $\mathrm{pC} 1 \mathrm{e}$ or other members of the $\mathrm{pC} 1$ cell type group. 


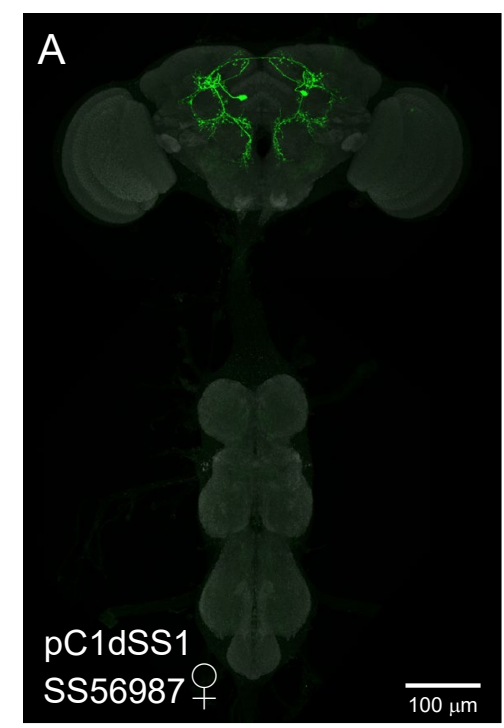

B
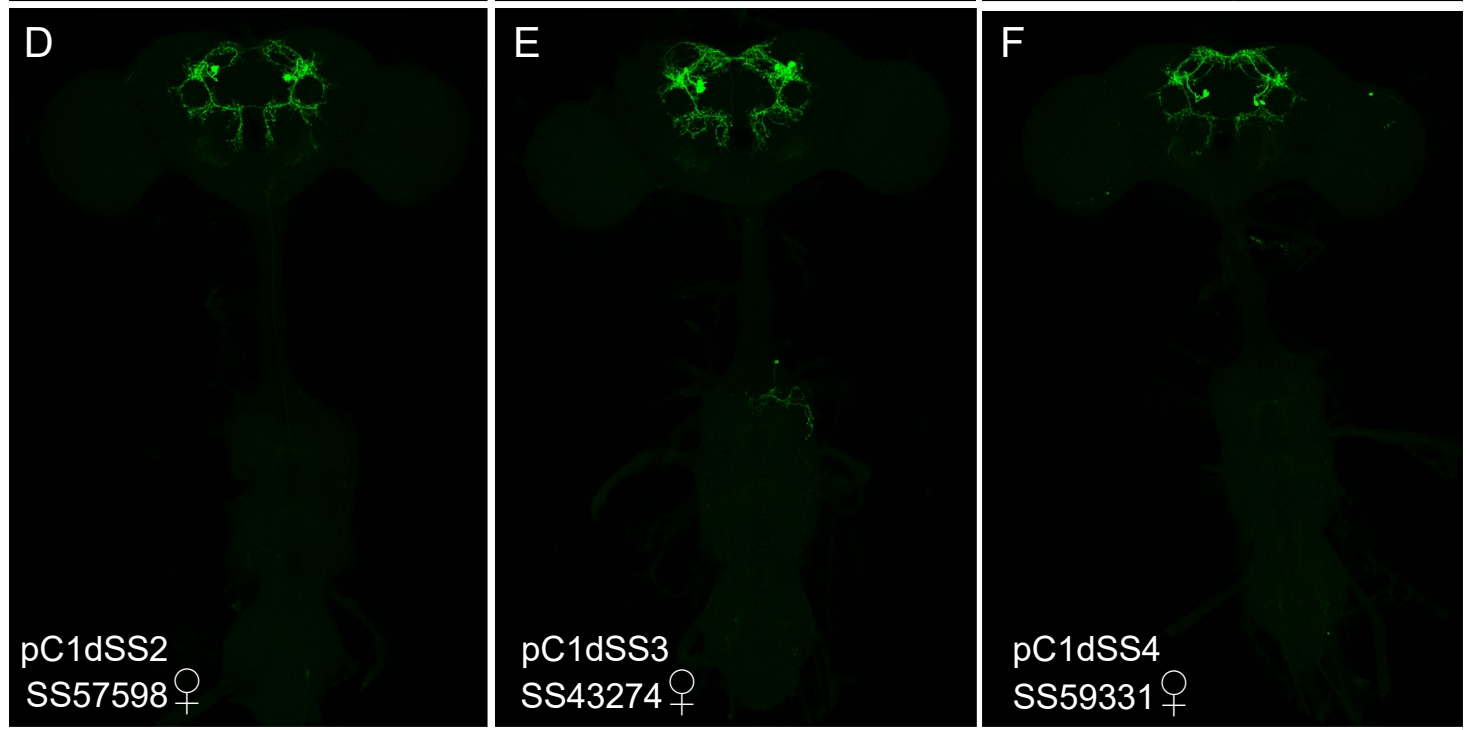

Figure 5 - figure supplement 1. Expression patterns of pC1d split-GAL4 lines. (A - F) 20X maximum intensity projections of the brains and ventral nerve cords of the indicated split-GAL4 lines crossed to 20xUAS-

CsChrimson::mVenus. As we are visualizing the optogenetic effector itself, these images serve as a way to compare the relative expression levels of the effector in the different split-GAL4 lines. Sex of the imaged brain is indicated.

The scale bar shown in A applies to panels A - F. ( $\left.D^{\prime}-F^{\prime}\right)$ Enlargements of the central brain of the images shown in D - F. (A - C) The SS56987 (pC1dSS1) expression pattern is shown; the neuropil reference channel shown in A (gray). Note that no expression in $\mathrm{pC} 1 \mathrm{~d}$ neurons is seen in the male nervous system which was imaged under identical conditions to the female nervous system shown in B. (D, D') Expression pattern of SS57598 (pC1dSS2). (E, E') Expression pattern of SS43274 (pC1dSS3). Note that this line expresses in both pC1d and pC1e. This line shows expression in males in a cell type clearly distinct from pC1d. Arrows indicate cell bodies. (F, F'). Expression pattern of SS59331 (pC1dSS4). Note that this line expresses in both pC1d and pC1e. Arrows indicate cell bodies. 
bioRxiv preprint doi: https://doi.org/10.1101/2020.05.27.118810; this version posted May 27, 2020. The copyright holder for this preprint (which

was not certified by peer review) is the author/funder, who has granted bioRxiv a license to display the preprint in perpetuity. It is made available under aCC-BY 4.0 International license.

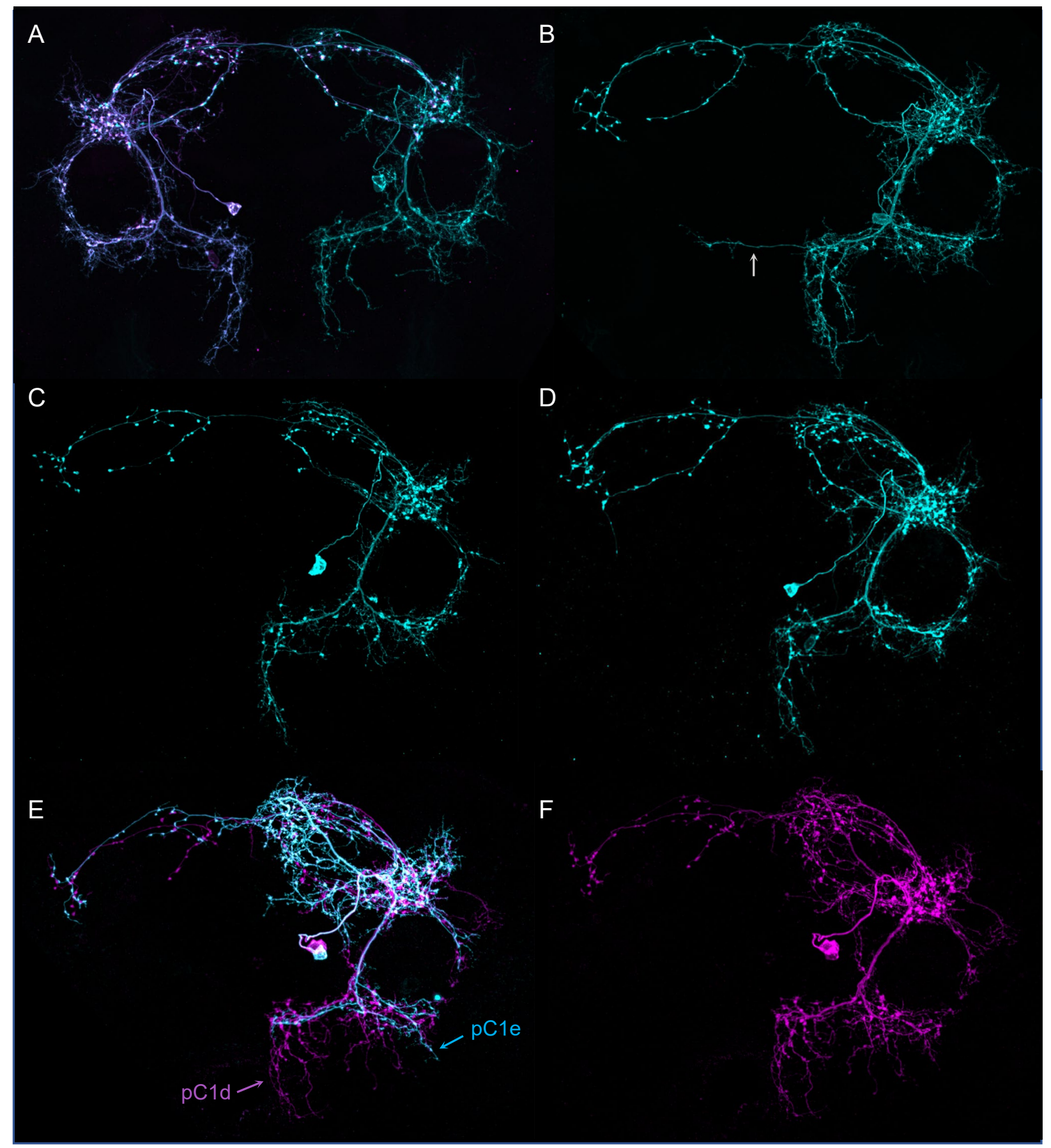

Figure 5 - figure supplement 2. Morphologies of individual pC1d neurons. The images in all panels were generated by stochastic labeling of pC1d split-GAL4 lines using the MultiColor FlipOut method (Nern et al. 2015). Both brain hemispheres are shown. (A - D) Images from SS56987 (pC1dSS1). The arrow in (B) highlights a process that crosses the midline which is often seen in pC1d cells; see ( $\left.D^{\prime}\right)$ and $\left(F^{\prime}\right)$ in Figure 5 for additional examples. (A) The right and left hemisphere pC1d cells are shown. (E, F) Images from SS43274 (pC1dSS3). 

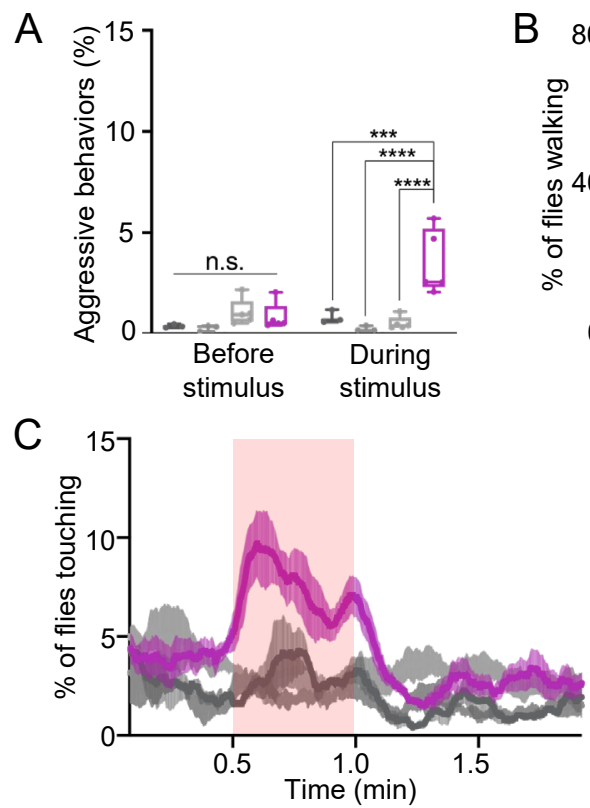

D

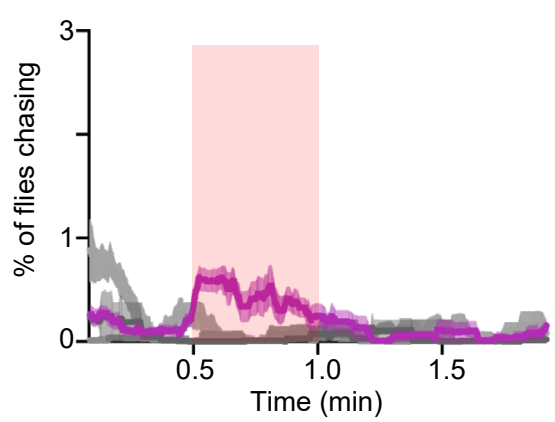

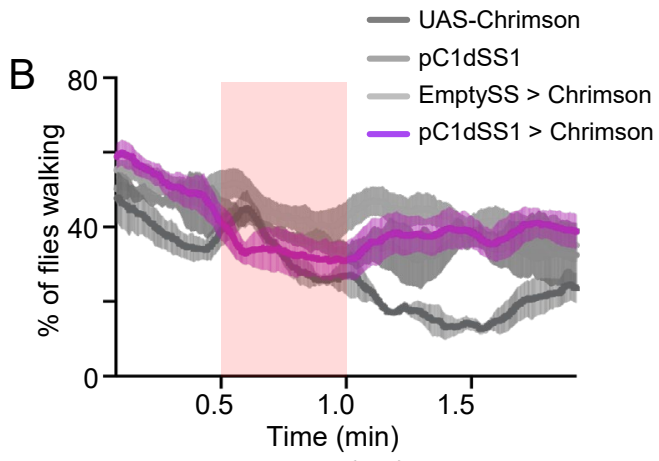

$\mathrm{C}^{\prime}$

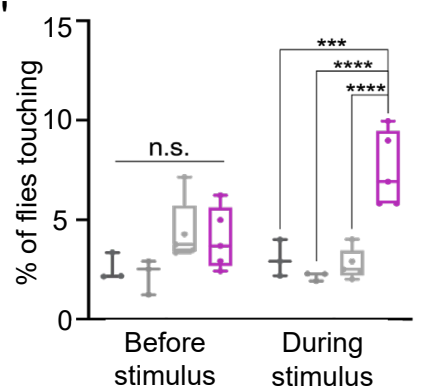

$D^{\prime}$

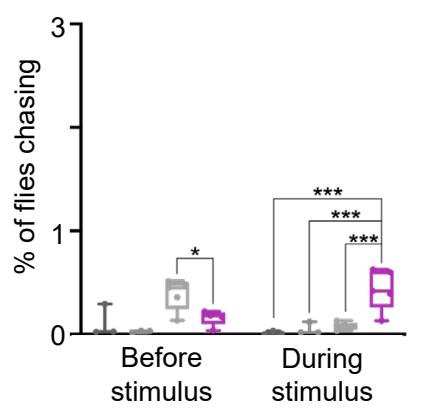

Figure 5 - figure supplement 3. Behavioral characterization of female flies after pC1d activation. Behaviors were measured over the course of a 2 -minute trial during which a 30 -second $0.4 \mathrm{~mW} / \mathrm{mm}^{2}$ continuous light stimulus was delivered (A) average percentage of flies engaging in aggressive behaviors over the 30 -second period prior to or during stimulus delivery. (B, C, D) Percentage of female flies walking (B), touching (C), or chasing (D). (C', $\mathrm{D}^{\prime}$ ) Average percentage of flies touching $\left(C^{\prime}\right)$ or chasing $\left(D^{\prime}\right)$ over the 30 -second period prior to or during the stimulus delivery in $\mathrm{C}$ or $\mathrm{D}$, respectively. Points represent separate experiments consisting of approximately 15 flies. 20xUAS-CsChrimson, $\mathrm{n}=3$ experiments; $\mathrm{pCldSS1}, \mathrm{n}=3$ experiments; EmptySS $>20 x \mathrm{xAS}-\mathrm{Cs}$ Chrimson, $\mathrm{n}=5$ experiments; pC1dSS1 > 20xUAS-CsChrimson, $\mathrm{n}=5$ experiments. Box-and-whisker plots show median and interquartile range (IQR); whiskers show range. A two-way ANOVA with a Tukey's multiple comparisons post-hoc test was used for statistical analysis. Asterisk indicates significance from $0:{ }^{*}<<0.05 ; * * * \mathrm{p}<0.001 ; * * * * \mathrm{p}<0.0001$; n.s., not significant. 

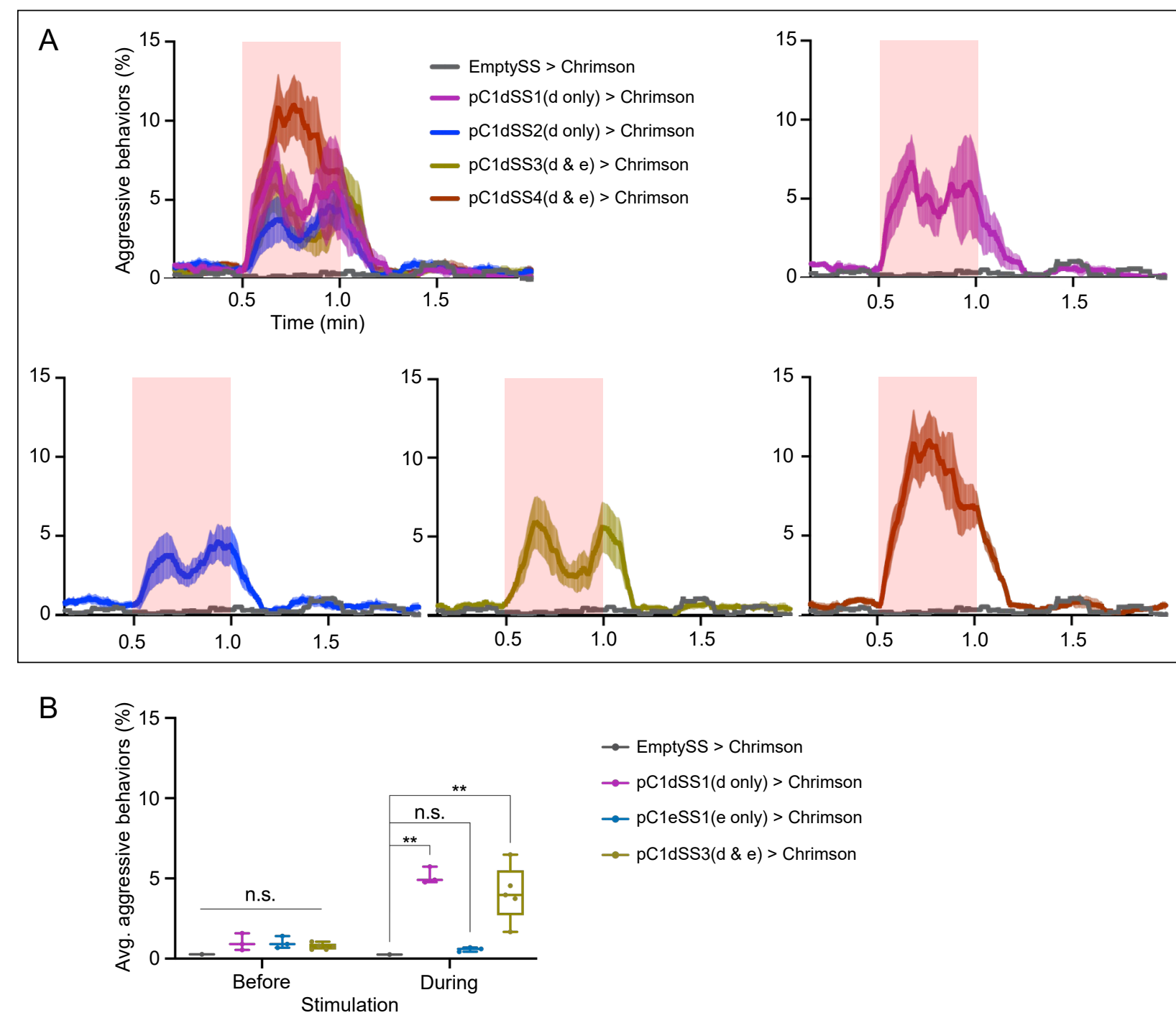

Figure 5 - figure supplement 4. Optogenetic activation of additional lines labeling pC1d split-GAL4 lines display similar behavioral results to pC1dSS1. (A) Percentage of flies engaging in aggressive behaviors over the course of a 2-minute trial during which a 30 -second $0.1 \mathrm{~mW} / \mathrm{mm}^{2}$ continuous light stimulus was delivered. Red shading indicates the stimulus period. The mean is represented as a solid line and error bars represent variation between experiments. Note that two pC1d split-GAL4 lines label just $\mathrm{pC} 1 \mathrm{~d}$ and two others label both $\mathrm{pC} 1 \mathrm{~d}$ and $\mathrm{pCle}$, as indicated. Individual curves are shown for comparison. Each experiment included approximately 15 flies. EmptySS $>$ Chrimson, $\mathrm{n}=1$ experiment; $\mathrm{pC} 1 \mathrm{dSS} 1>$ Chrimson, $\mathrm{n}=3$ experiments; $\mathrm{pC} 1 \mathrm{dSS} 2>$ Chrimson, $\mathrm{n}=6$ experiments; $\mathrm{pC} 1 \mathrm{dSS} 3>$ Chrimson, $\mathrm{n}=5$ experiments; $\mathrm{pC} 1 \mathrm{dSS} 4>$ Chrimson, $\mathrm{n}=5$ experiments. (B) Average percentage of flies engaging in aggressive behaviors over the 30 second period prior to or during the delivery of a $0.1 \mathrm{~mW} / \mathrm{mm}^{2}$ stimulus. Points represent separate experiments consisting of approximately 15 flies. EmptySS > Chrimson, $\mathrm{n}=1$ experiment; $\mathrm{pC} 1 \mathrm{dSS} 1>$ Chrimson, $\mathrm{n}=3$ experiments; $\mathrm{pC} 1 \mathrm{eSS} 1>$ Chrimson, $\mathrm{n}=3$ experiments; pC1dSS3 $>$ Chrimson, $n=5$ experiments. Box-and-whisker plots show median and interquartile range (IQR); whiskers show range. A two-way ANOVA with a Tukey's multiple comparisons post-hoc test was used for statistical analysis. Asterisk indicates significance from $0: * * p<0.01 ;$ n.s., not significant. 

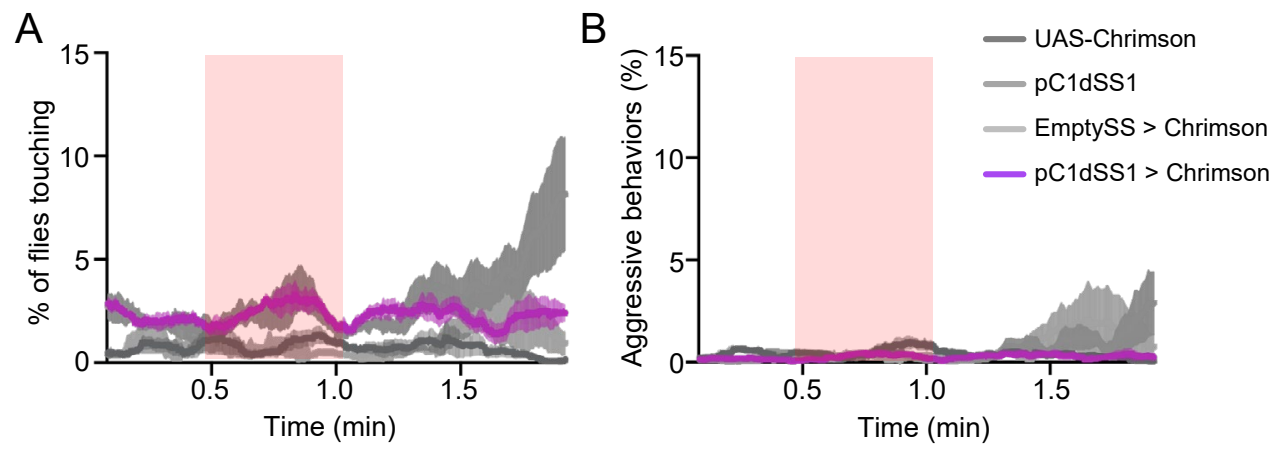

Figure 5 - figure supplement 5. Optogenetic stimulation of pC1dSS1>Chrimson males does not result in aggressive behavior. (A - B) Percentage of male flies touching (A) or performing aggressive behaviors (B) over the course of a 2-minute trial during which a 30 second $0.4 \mathrm{~mW} / \mathrm{mm}^{2}$ continuous light stimulus was delivered. The mean is represented as a solid line and error bars represent variation between experiments. Each experiment included approximately 15 flies. 20xUAS-CsChrimson, $\mathrm{n}=5$ experiments; $\mathrm{pC} 1 \mathrm{dSS} 1, \mathrm{n}=4$ experiments; EmptySS $>20 x U A S-C s C h r i m s o n, ~ n=3$ experiments; $\mathrm{pC} 1 \mathrm{dSS} 1>20 x U A S-C s C h r i m s o n, \mathrm{n}=5$ experiments. 

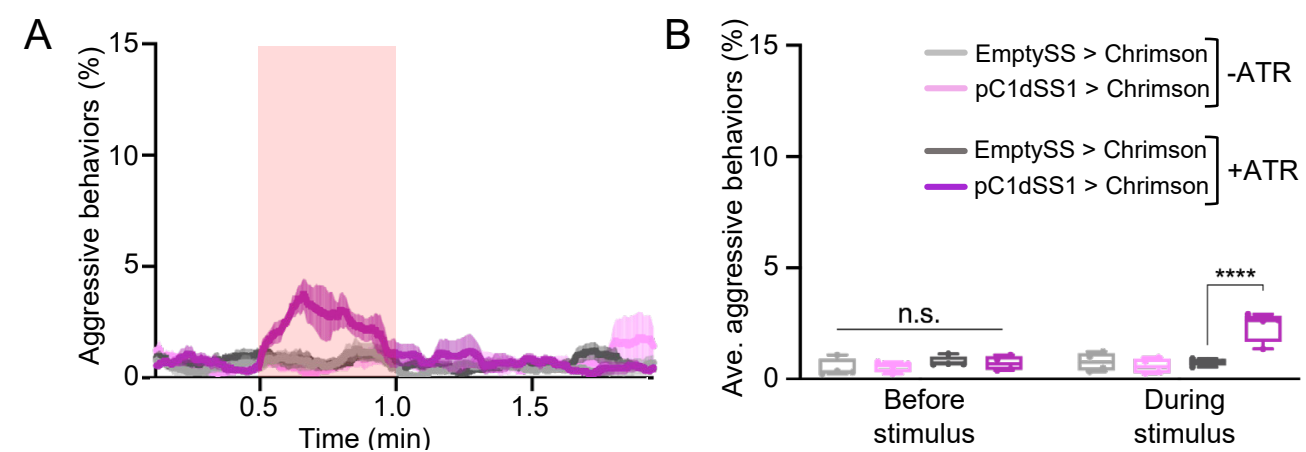

Figure 5 - figure supplement 6. Optogenetic activation of aggression depends on feeding all trans-retinal. (A) Percentage of flies that engaged in aggressive behaviors. Flies were raised with food supplemented with all transretinal (+ATR) or not (-ATR). A 30-second $0.1 \mathrm{~mW} / \mathrm{mm}^{2}$ continuous light stimulus was delivered 30 sec after the start of a 2-minute trial as indicated by the red shading. The mean is represented as a solid line and error bars represent variation between experiments. Each experiment included approximately 15 flies. (B) Average percentage of flies engaging in aggressive behaviors over the 30 -second period prior to or during the stimulus delivery in A. Points represent separate experiments consisting of approximately 15 flies. +ATR: EmptySS $>$ Chrimson, $n=4$ experiments; pC1dSS1 > Chrimson, $\mathrm{n}=4$ experiments; -ATR: EmptySS > Chrimson, $\mathrm{n}=4$ experiments; $\mathrm{pC} 1 \mathrm{dSS} 1>$ Chrimson, $\mathrm{n}=4$ experiments. Box-and-whisker plots show median and interquartile range (IQR); whiskers show range. A two-way ANOVA with a Tukey's multiple comparisons post hoc test was used for statistical analysis. Asterisk indicates significance from 0 : $* * * * p<0.0001$. 


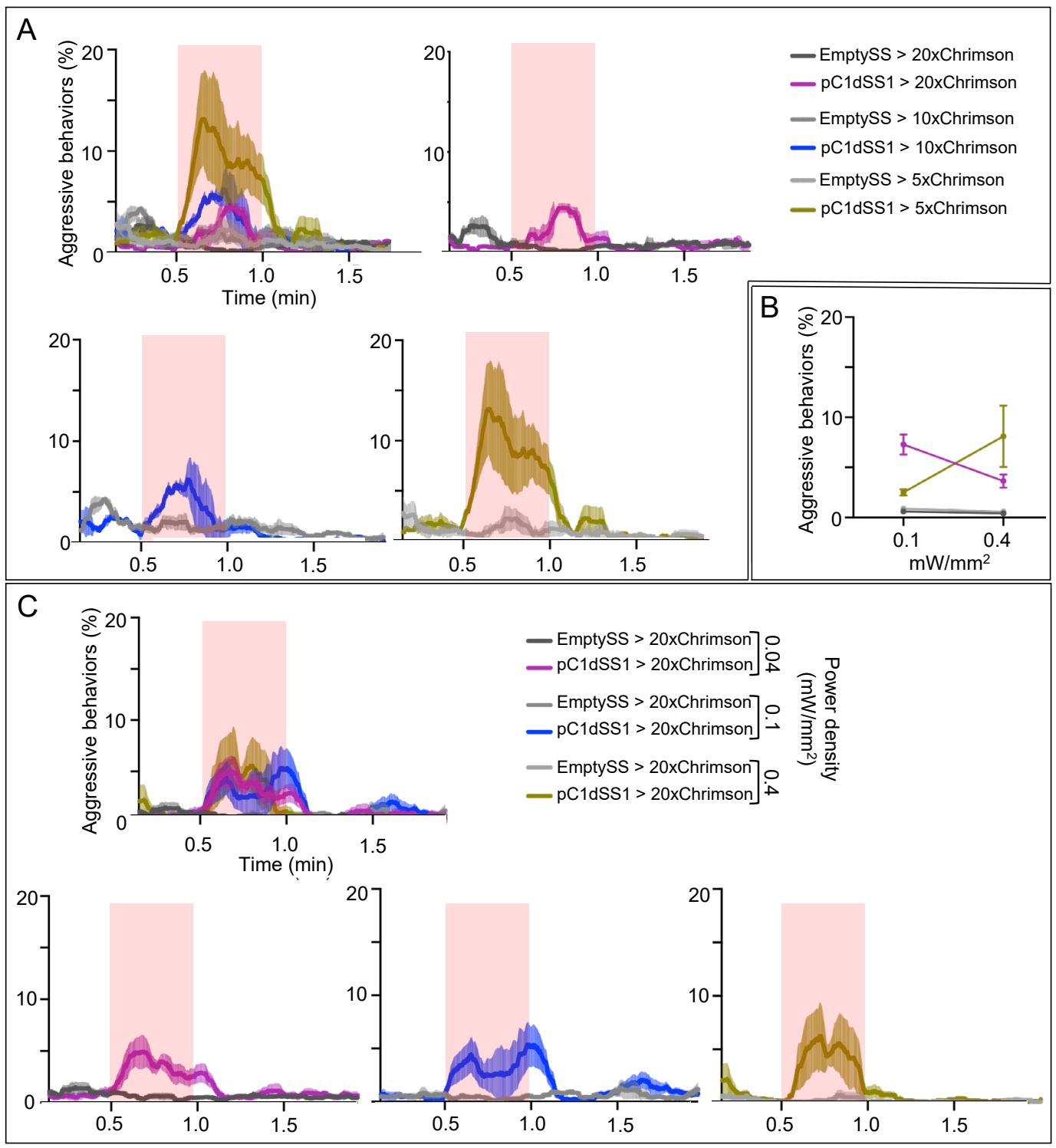

Figure 5 - figure supplement 7 . Behavioral effects of stimulus delivery and effector strength. (A) Percentage of flies engaging in aggressive behaviors over the course of a 2-minute trial during which a 30 -second $0.1 \mathrm{~mW} / \mathrm{mm}^{2}$ continuous light stimulus was delivered to flies carrying 5XUAS, 10xUAS, and 20xUAS effectors. Individual curves are shown for comparison and each experiment included approximately 15 flies. EmptySS $>20 x U A S-$ Chrimson, $\mathrm{n}=2$ experiments; $\mathrm{pC} 1 \mathrm{dSS} 1>20 \mathrm{xUAS}-$ Chrimson, $\mathrm{n}=2$ experiments; EmptySS $>10 x \mathrm{xAS}-$ Chrimson, $\mathrm{n}$ $=2$ experiments; $\mathrm{pC} 1 \mathrm{dSS} 1>10 \mathrm{xUAS}-$ Chrimson, $\mathrm{n}=2$ experiments; EmptySS $>5 \mathrm{xUAS}$-Chrimson, $\mathrm{n}=3$ experiments; $\mathrm{pC} 1 \mathrm{dSS} 1>5 \mathrm{xUAS}$-Chrimson, $\mathrm{n}=3$ experiments. (B) Average percentage of flies engaging in aggressive behaviors over the 30 -second period during stimulus delivery. Each experiment included approximately 15 flies. $0.1 \mathrm{~mW} / \mathrm{mm}^{2}$ : EmptySS $>5 x$ UAS-Chrimson (light gray), $\mathrm{n}=6$ experiments; $\mathrm{pC} 1 \mathrm{dSS} 1>5 \mathrm{xUAS}-\mathrm{Chrimson}$ (gold), $\mathrm{n}=6$ experiments; EmptySS $>20 x$ UAS-Chrimson (dark gray), $\mathrm{n}=6$ experiments; $\mathrm{pC} 1 \mathrm{dSS} 1>20 \mathrm{xUAS}-$ Chrimson (purple), $\mathrm{n}=7$ experiments; $0.4 \mathrm{~mW} / \mathrm{mm}^{2}$ : EmptySS $>5 \mathrm{xUAS}$-Chrimson, $\mathrm{n}=6$ experiments; $\mathrm{pC} 1 \mathrm{dSS} 1>$ 5xUAS-Chrimson, $\mathrm{n}=2$ experiments; EmptySS $>20 x U A S-C h r i m s o n, ~ n=3$ experiments; $p C 1 d S S 1>20 x U A S-$ Chrimson, $\mathrm{n}=5$ experiments. Bars are mean + - S.E.M. (C) Percentage of flies engaging in aggressive behaviors over the course of a 2-minute trial during which a 30 -second $0.4,0.1$, or $0.04 \mathrm{~mW} / \mathrm{mm}^{2}$ continuous light stimulus was delivered. Individual curves are shown for comparison and each experiment included approximately 15 flies. $0.04 \mathrm{~mW} / \mathrm{mm}^{2}$ : EmptySS $>$ Chrimson, $\mathrm{n}=5$ experiments; $\mathrm{pC} 1 \mathrm{dSS} 1>$ Chrimson, $\mathrm{n}=5$ experiments; $0.1 \mathrm{~mW} / \mathrm{mm}^{2}$ : EmptySS $>$ Chrimson, $\mathrm{n}=4$ experiments; $\mathrm{pC} 1 \mathrm{dSS} 1>$ Chrimson, $\mathrm{n}=4$ experiments; $0.4 \mathrm{~mW} / \mathrm{mm}^{2}$ : EmptySS > Chrimson, $\mathrm{n}=3$ experiments; $\mathrm{pC} 1 \mathrm{dSS} 1>$ Chrimson, $\mathrm{n}=3$ experiments. 
A

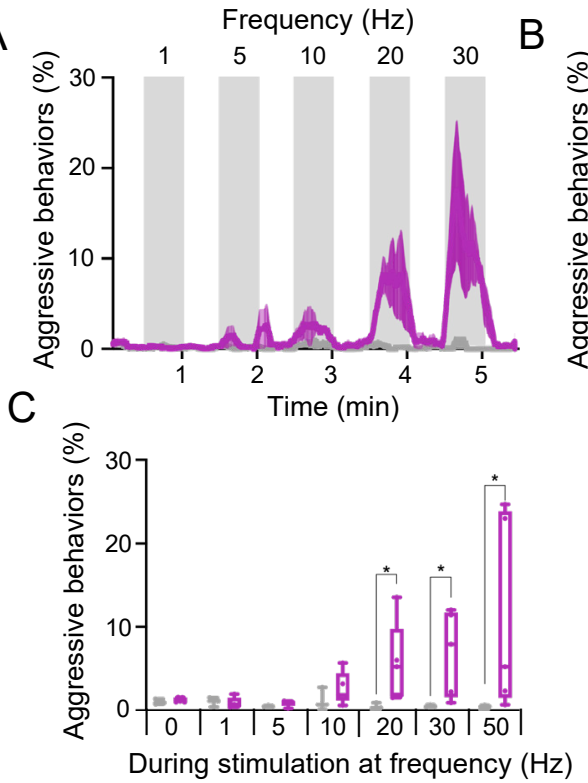

$B$
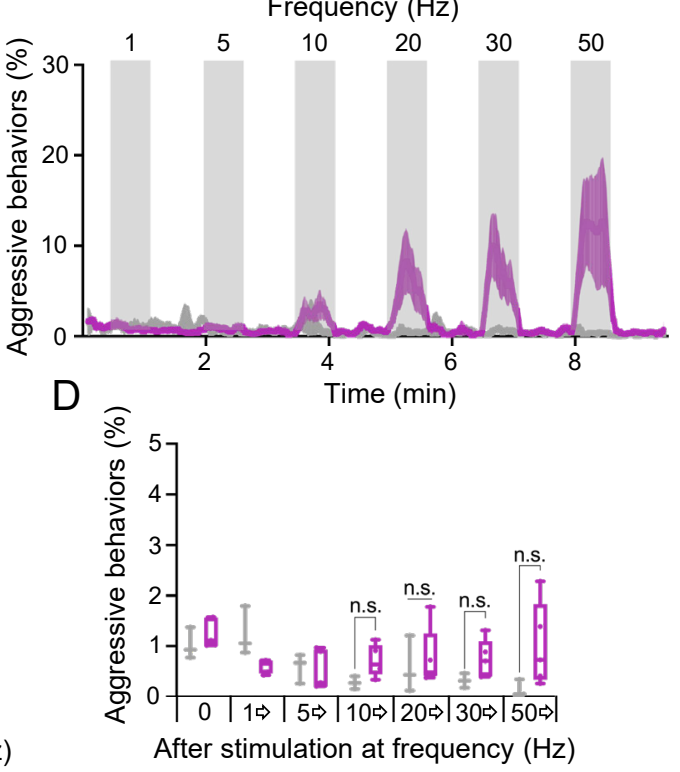

Figure 5 - figure supplement 8. Behavioral effects of the frequency of optogenetic stimulation. (A - B) Blocks of 30-second photostimulation (grey bars) with increasing stimulation frequency separated by 30 (A) or 60 (B) second intervals were delivered sequentially to pC1dSS1 females. Light was delivered at $0.1 \mathrm{~mW} / \mathrm{mm}^{2}$ with at a 10 ms pulse width, but with increasing pulse frequency. Numbers above the grey bars correspond to frequency over the 30 -second stimulus period. The pulse period and pulse number during each period was as follows: $1000 \mathrm{~ms}, 30$; $200 \mathrm{~ms}, 150 ; 100 \mathrm{~ms}, 300 ; 50 \mathrm{~ms}, 600 ; 33 \mathrm{~ms}, 909 ; 20 \mathrm{~ms}, 1500$. pC1dSS1 > 20xUAS-Chrimson is shown in purple; EmptySS $>20 x U A S-C h r i m s o n$ is shown in gray. The mean is represented as a solid line and shaded error bars represent variation between experiments. Each experiment included approximately 15 flies. A: EmptySS > 20xUAS-Chrimson, $\mathrm{n}=3$ experiments; $\mathrm{pC} 1 \mathrm{dSS} 1>20 x \mathrm{x}$ AS-Chrimson, $\mathrm{n}=3$ experiments; B: EmptySS > 20xUASChrimson, $\mathrm{n}=3$ experiments; $\mathrm{pC} 1 \mathrm{dSS} 1>20 \mathrm{xUAS}-\mathrm{Chrimson}, \mathrm{n}=5$ experiments. (C) Average percentage of flies performing aggressive behaviors over the 30 -second period during stimulus delivery in B. (D) Average percentage of flies performing aggressive behaviors over the 30 -second period before $1 \mathrm{~Hz}$, the 60 -second periods between subsequent stimuli, and the 30 -seconds after $50 \mathrm{~Hz}$ stimulation in B. Points indicate separate experiments. Box-andwhisker plots show median and interquartile range (IQR); whiskers show range. Mann-Whitney $U$-tests were used for statistical analysis. Asterisk indicates significance from 0 : ${ }^{*} \mathrm{p}<0.05$; n.s., not significant. 

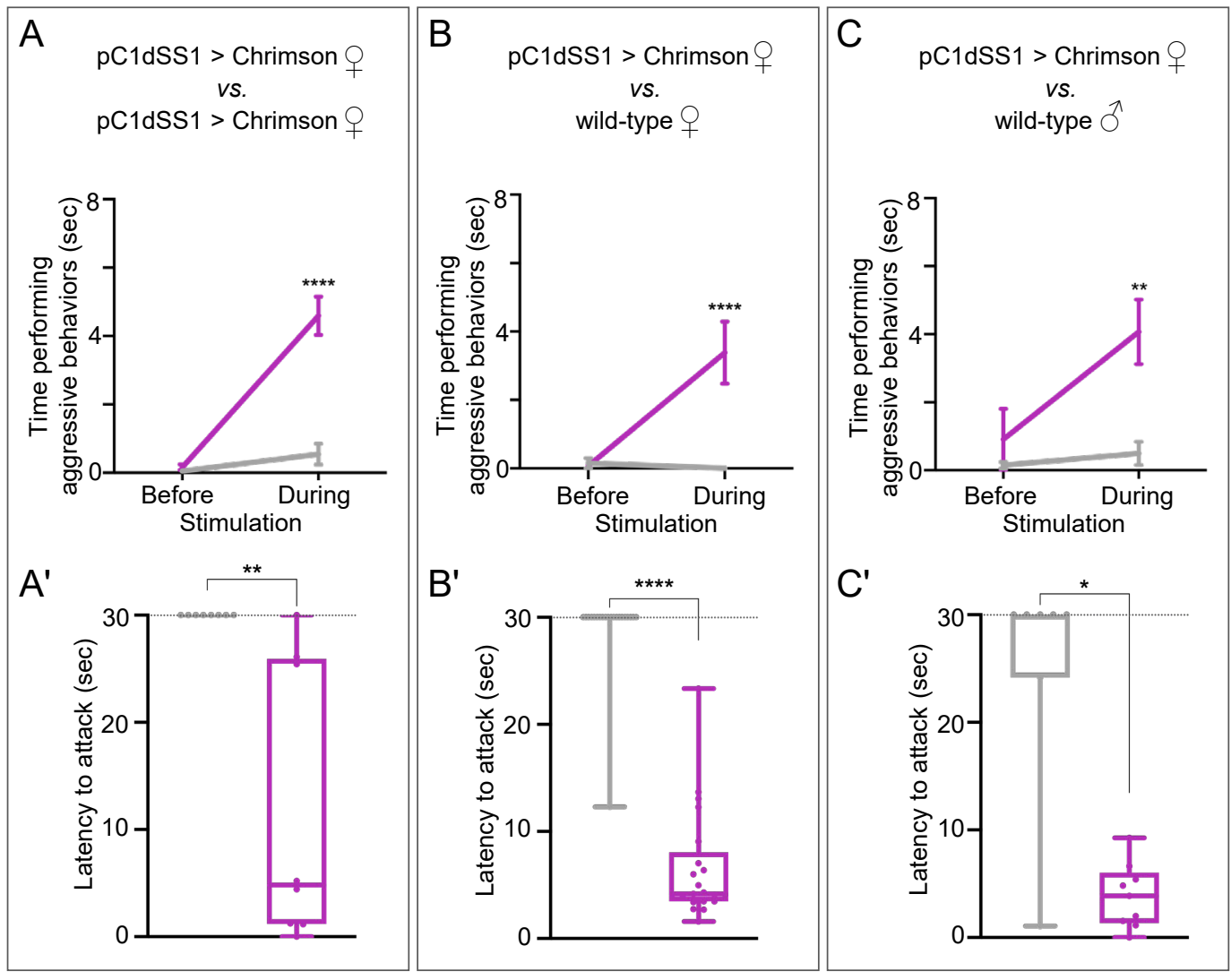

Figure 5 - figure supplement 9. pC1d activation also increases aggression against wild-type females and males. (A - C) Total time an individual spent performing aggressive behaviors over the 30 second period prior to or during stimulation, see Figure 3 legend for detail. $\left(\mathrm{A}^{\prime}-\mathrm{C}^{\prime}\right)$ Amount of time during a 30 second $0.1 \mathrm{~mW} / \mathrm{mm}^{2}$ continuous stimulation period until first aggressive encounter. Points indicate individual flies. Dotted lines at $30 \mathrm{sec}$ indicate the end of the trial and error bars are mean +/- S.E.M. A: EmptySS $>20 x U A S-C h r i m s o n, ~ n=22$ flies; pC1dSS1 > 20xUAS-Chrimson, $\mathrm{n}=22$ flies; B: EmptySS > 20xUAS-Chrimson, $\mathrm{n}=8$ flies; $\mathrm{pC} 1 \mathrm{dSS} 1>20 \mathrm{xUAS}-$ Chrimson, $\mathrm{n}=8$ flies; C: EmptySS $>20 x U A S-C h r i m s o n, ~ n=7$ flies; $\mathrm{pCldSS} 1>20 \mathrm{xUAS}-$ Chrimson, $\mathrm{n}=8$ flies. Box-and-whisker plots show median and interquartile range (IQR); whiskers show either $1.5 \times \mathrm{IQR}$ of the lower and upper quartiles. A two-way ANOVA with a Sidak's multiple comparisons post-hoc test (A $-\mathrm{C}$ ) or Mann-Whitney $U$ $\left(\mathrm{A}^{\prime}-\mathrm{C}^{\prime}\right)$ post hoc test was used for statistical analysis. Asterisk indicates significance from $0:{ }^{*} \mathrm{p}<0.05 ;{ }^{* *} \mathrm{p}<0.01$; $* * * * \mathrm{p}<0.0001$. 
bioRxiv preprint doi: https://doi.org/10.1101/2020.05.27.118810; this version posted May 27, 2020. The copyright holder for this preprint (which

was not certified by peer review) is the author/funder, who has granted bioRxiv a license to display the preprint in perpetuity. It is made available under aCC-BY 4.0 International license.

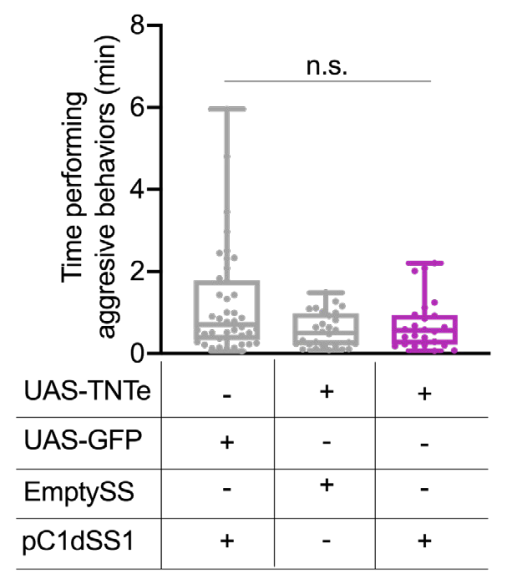

Figure 5 - figure supplement 10. pC1d inactivation did not significantly diminish aggressive behavior. Total time an individual spent performing aggressive behaviors over a 30-minute trial. Points indicate individual flies. pC1dSS1 > UAS-GFP, $n=42$ flies; EmptySS > UAS-TNTe, $n=28$ flies; pC1dSS1 > UAS-TNTe, $n=24$ flies. Box-and-whisker plots show median and interquartile range (IQR); whiskers show either $1.5 \times$ IQR of the lower and upper quartiles. Kruskal-Wallis and Dunn's post hoc tests were used for statistical analysis. n.s., not significant. 

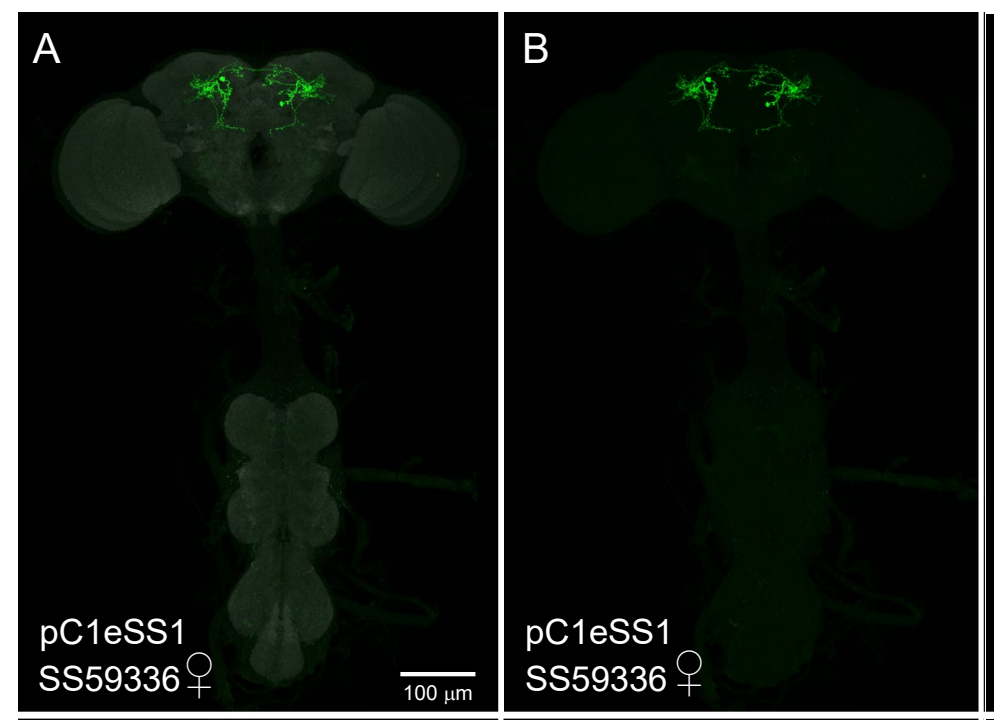

Figure 6 - figure supplement 1. Expression patterns of pC1e split-GAL4 lines. (A - E) 20X maximum intensity projections of the brains and ventral nerve cords of the indicated split-GAL4 lines crossed to 20xUAS-

CsChrimson::mVenus. Because we are visualizing the optogenetic effector itself, these images serve as a way to compare the relative expression levels of the effector in the different split-GAL4 lines. The gender of the imaged brain is indicated. The scale bar shown in A applies to panels A-E. (D', E') Enlargements of the central brain of the images shown in D and E. (A - C) The SS59336 (pC1eSS1) expression pattern is shown; the neuropil reference channel is shown in A (gray). Note that no expression in aIPg neurons is seen in the male nervous system (C) which was imaged under identical conditions to the female nervous system shown in B. (D, D') Expression pattern of SS39313 (pC1eSS2). This line shows expression in males in a cell type clearly distinct from pC1e (not shown). (E, E') Expression pattern of SS59433 (pC1eSS3). 


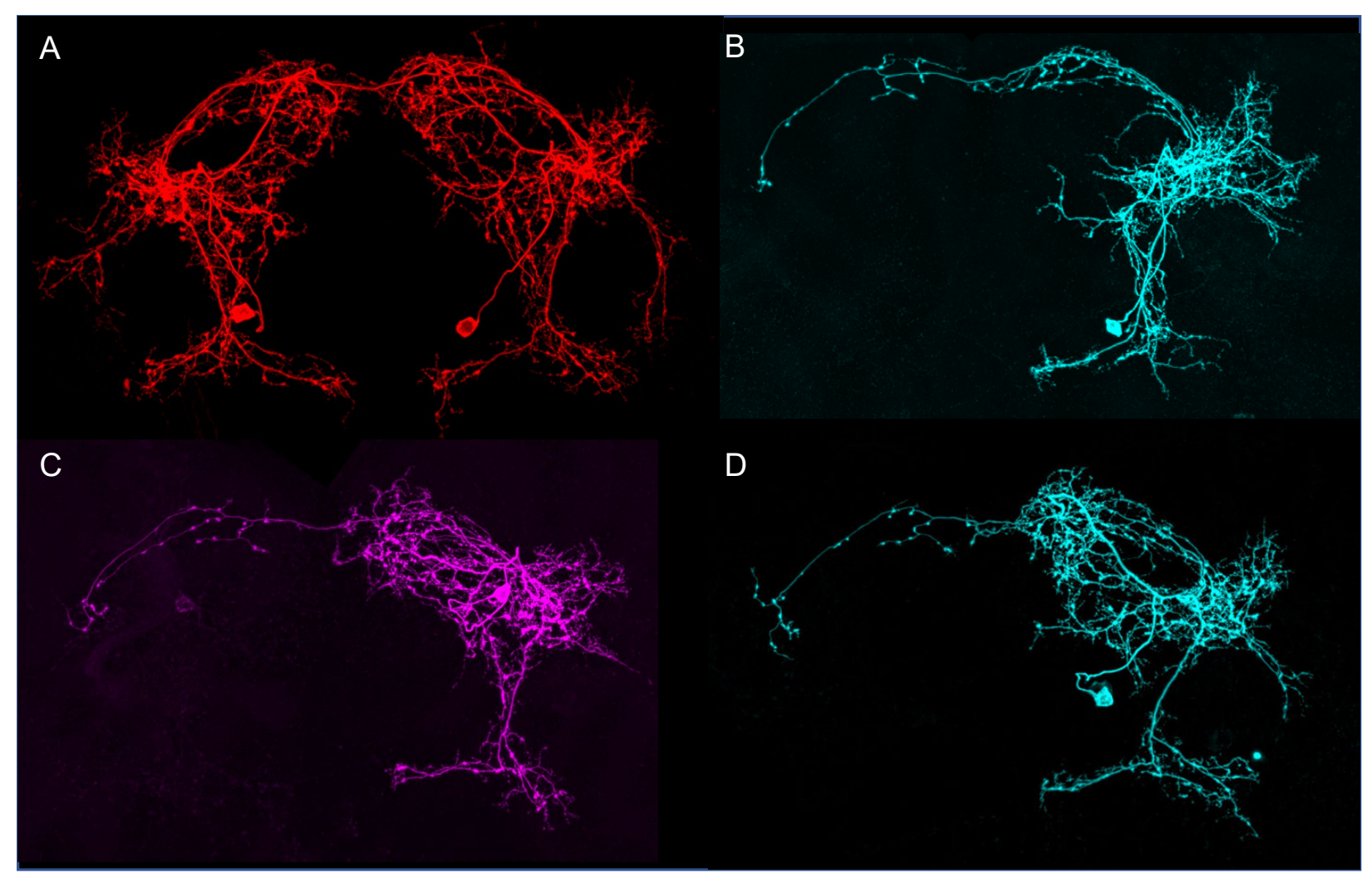

Figure 6 - figure supplement 2. Morphologies of individual pC1e neurons. The images in all panels were generated by stochastic labeling of pC1e split-GAL4 lines using the MultiColor FlipOut method (Nern et al. 2015). (A, B) Images from SS59336 (pC1eSS1). (A) The right and left hemisphere pC1e cells are shown. (C, D) Images from SS43274 (pC1dSS3). Note this line contains both pC1d and pC1e cells. See Figure 5 - figure supplement 2 for pCld cells. 


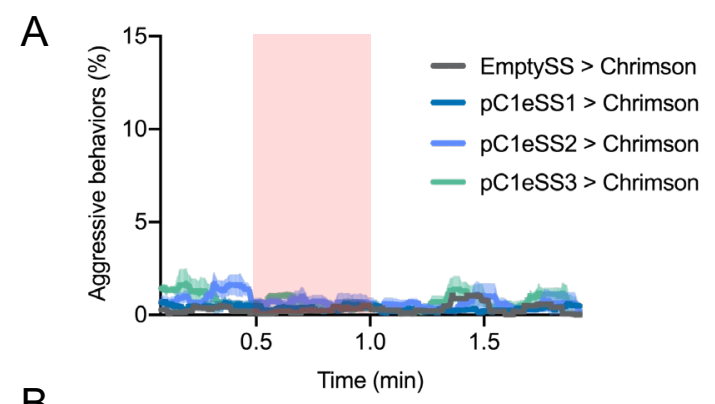

B

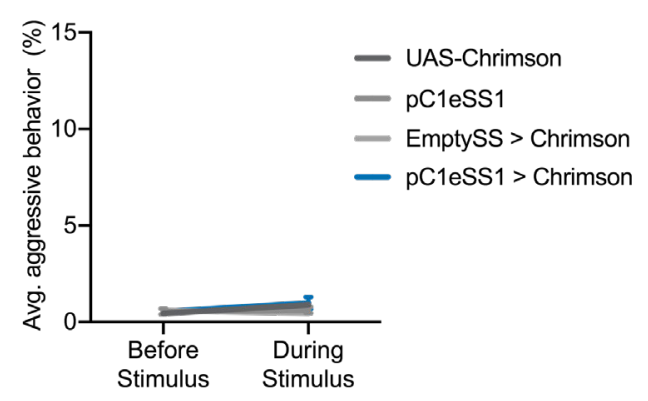

Figure 6 - figure supplement 3. Optogenetic activation of additional lines labeling pC1e. (A) Percentage of flies engaging in aggressive behaviors over the course of a 2-minute trial during which a 30 -second $0.1 \mathrm{~mW} / \mathrm{mm}^{2}$ continuous light stimulus was delivered. Red shading indicates the stimulus period. The mean is represented as a solid line and shaded error bars represent variation between experiments. Each experiment included approximately 15 flies. EmptySS $>$ Chrimson, $n=1$ experiment; $\mathrm{pC} 1 \mathrm{eSS} 1>$ Chrimson, $\mathrm{n}=3$ experiments; $\mathrm{pC} 1 \mathrm{eSS} 2>\mathrm{Chrimson}, \mathrm{n}$ $=3$ experiments; $\mathrm{pCleSS} 3>$ Chrimson, $\mathrm{n}=3$ experiments. (B) Average percentage of flies engaging in aggressive behaviors over the 30 second period prior to or during the delivery of a $0.1 \mathrm{~mW} / \mathrm{mm}^{2}$ stimulus. Each experiment included approximately 15 flies. 20xUAS-CsChrimson, $\mathrm{n}=3$ experiments; $\mathrm{pC} 1 \mathrm{eSS} 1, \mathrm{n}=2$ experiments; EmptySS > 20xUAS-CsChrimson, $\mathrm{n}=3$ experiments; pC1eSS1 > 20xUAS-CsChrimson, $\mathrm{n}=3$ experiments. Bars are mean $+/-$ S.E.M. 
A

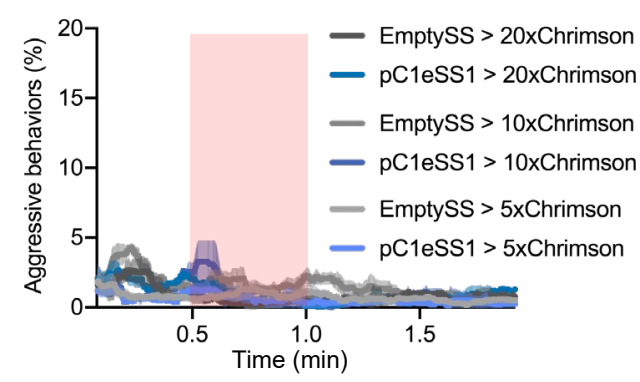

C

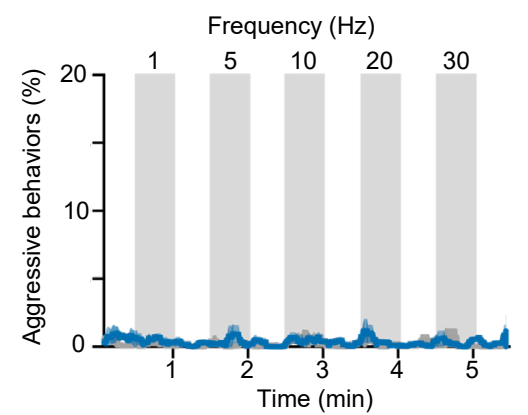

B

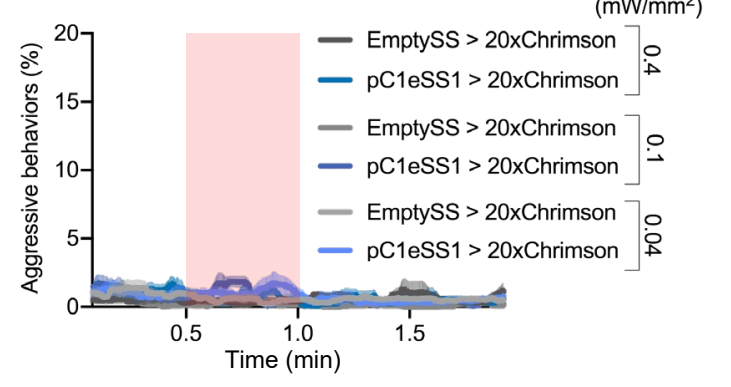

D

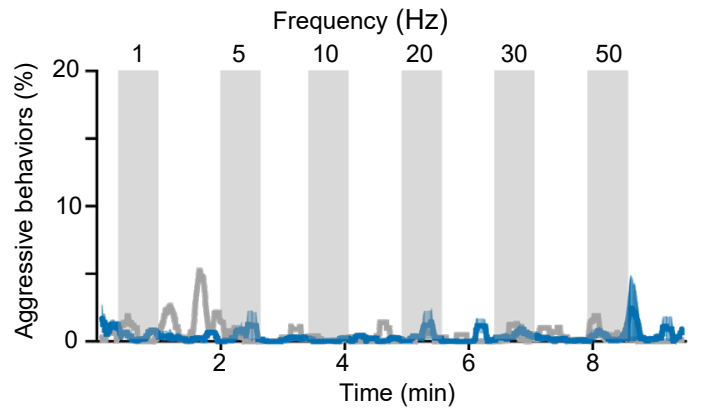

Figure 6 - figure supplement 4 . Behavioral effects of stimulus delivery and effector copy number. (A) Percentage of flies engaging in aggressive behaviors over the course of a 2-minute trial during which a 30-second $0.1 \mathrm{~mW} / \mathrm{mm}^{2}$ continuous light stimulus was delivered to flies carrying either 5xUAS, 10XUAS or 20xUAS effectors. Each experiment included approximately 15 flies. EmptySS $>20 x U A S-C h r i m s o n, ~ n=2$ experiments; pC1eSS1 > 20xUAS-Chrimson, $\mathrm{n}=2$ experiments; EmptySS > 10xUAS-Chrimson, $\mathrm{n}=3$ experiments; $\mathrm{pCleSS} 1>$ 10xUAS-Chrimson, $\mathrm{n}=2$ experiments; EmptySS $>5 \mathrm{xUAS}$-Chrimson, $\mathrm{n}=6$ experiments; $\mathrm{pCleSS} 1>5 \mathrm{xUAS}$ Chrimson, $\mathrm{n}=2$ experiments. (B) Percentage of flies engaging in aggressive behaviors over the course of a 2-minute trial during which a 30 -second $0.4,0.1$, or $0.04 \mathrm{~mW} / \mathrm{mm}^{2}$ continuous light stimulus was delivered. Each experiment included approximately 15 flies. $0.04 \mathrm{~mW} / \mathrm{mm}^{2}$ : EmptySS $>$ Chrimson, $\mathrm{n}=5$ experiments; $\mathrm{pCleSS} 1>$ Chrimson, $\mathrm{n}$ $=5$ experiments; $0.1 \mathrm{~mW} / \mathrm{mm}^{2}$ : EmptySS $>$ Chrimson, $\mathrm{n}=4$ experiments; $\mathrm{pCleSS} 1>$ Chrimson, $\mathrm{n}=3$ experiments; $0.4 \mathrm{~mW} / \mathrm{mm}^{2}$ : EmptySS $>$ Chrimson, $\mathrm{n}=3$ experiments; $\mathrm{pCleSS} 1>$ Chrimson, $\mathrm{n}=3$ experiments. $(\mathrm{C}-\mathrm{D})$ Blocks of 30-second photostimulation (grey bars) with increasing stimulation frequency separated by 30 (C) or 60 (D) second intervals were delivered sequentially to females. Light was delivered at $0.1 \mathrm{~mW} / \mathrm{mm}^{2}$ with a $10 \mathrm{~ms}$ pulse width and increasing frequencies resulting in higher numbers of pulses given over the 30 -second stimulus on period. The pulse period and pulse number during each period was as follows: $1000 \mathrm{~ms}, 30 ; 200 \mathrm{~ms}, 150 ; 100 \mathrm{~ms}, 300 ; 50 \mathrm{~ms}$, $600 ; 33 \mathrm{~ms}, 909 ; 20 \mathrm{~ms}, 1500$. Each experiment included approximately 15 flies. A: EmptySS > 20xUAS-Chrimson,

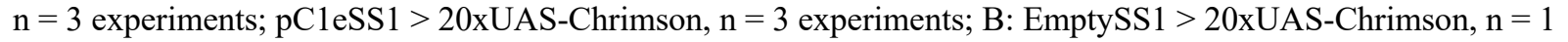
experiment; $\mathrm{pCleSS} 1>20 \mathrm{xUAS}-$ Chrimson, $\mathrm{n}=2$ experiments. 

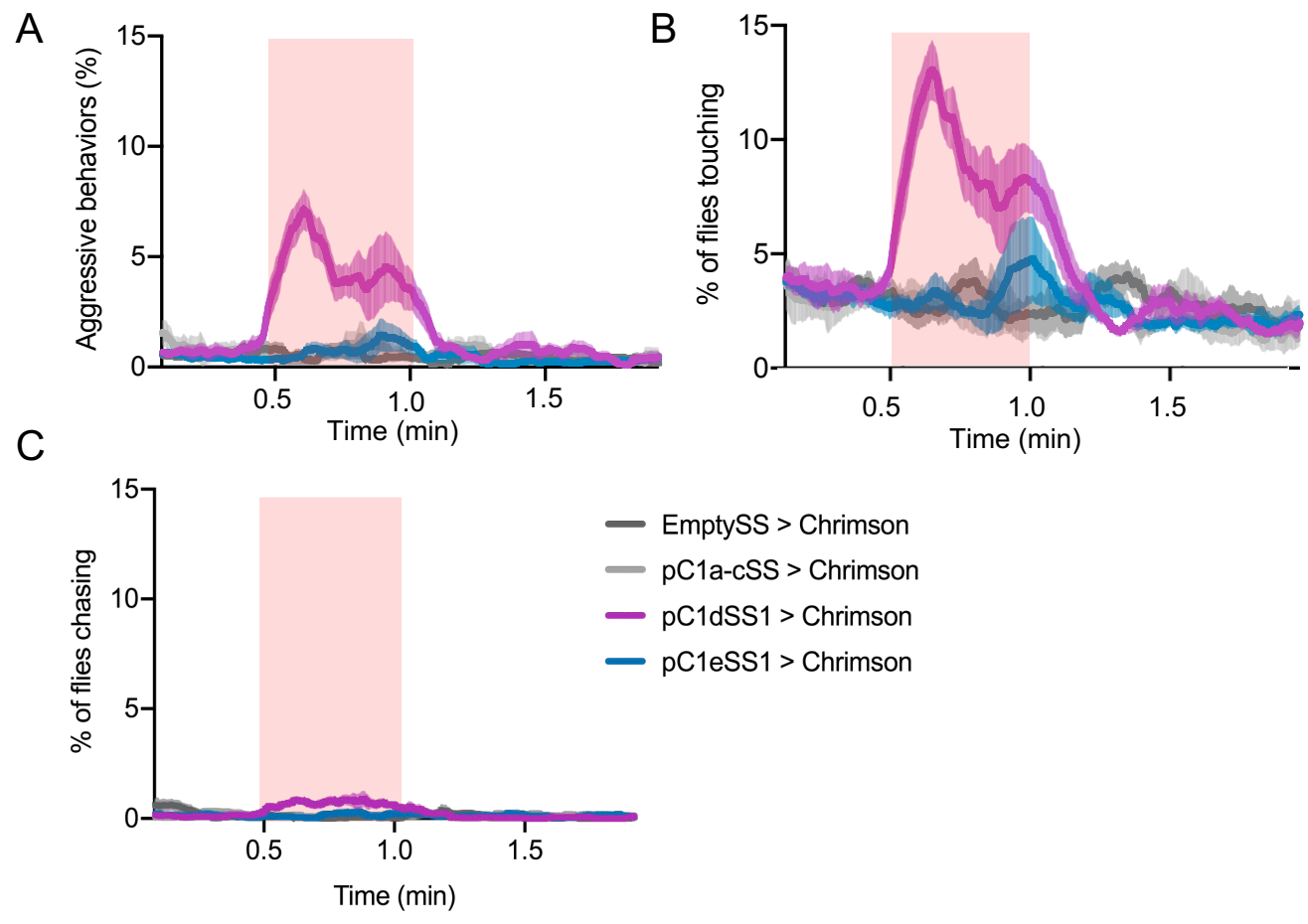

Figure 6 - figure supplement 5. Comparison of activation phenotypes of pC1d, pC1e and pC1a-c. (A-C)

Percentage of female flies engaged in aggressive behaviors (A) touching (B) or chasing (C) over the course of a twominute trial during which a 30 -second $0.1 \mathrm{~mW} / \mathrm{mm}^{2}$ continuous light stimulus was delivered (red shading). Each experiment included approximately 15 flies. EmptySS $>20 x U A S-C h r i m s o n, ~ n=4$ experiments; pC1a-cSS > 20xUAS-Chrimson, $\mathrm{n}=4$ experiments; $\mathrm{pCldSS} 1>20 \mathrm{xUAS}$-Chrimson, $\mathrm{n}=6$ experiments; $\mathrm{pCleSS} 1>20 \mathrm{xUAS}$ Chrimson, $\mathrm{n}=6$ experiments. The $\mathrm{pCla}-\mathrm{c}$ line was provided by $\mathrm{K}$. Wang and Barry Dickson. 
A

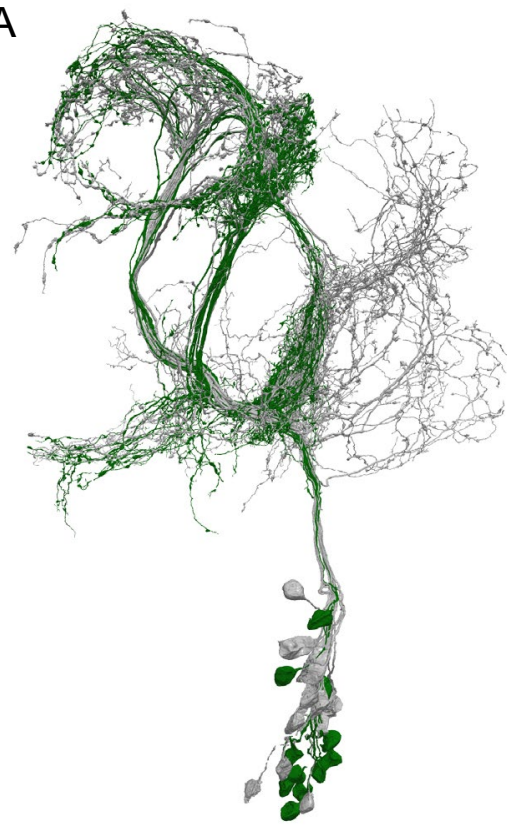

B

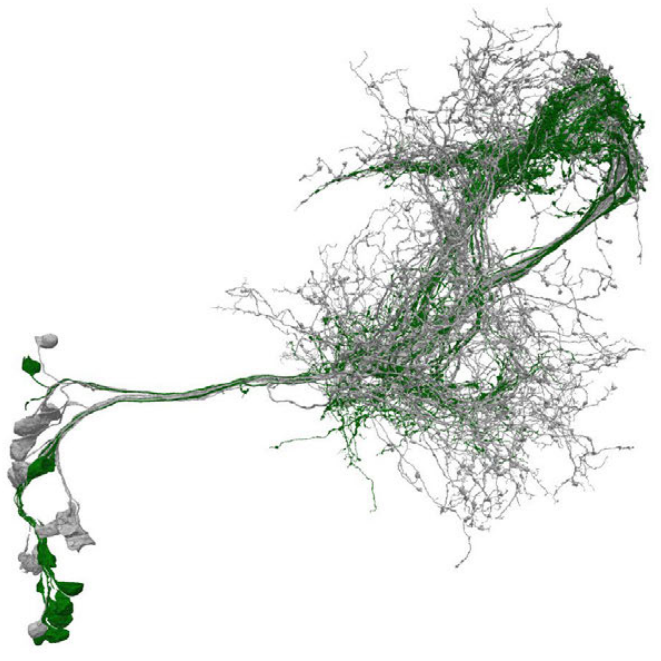

Figure 7 - figure supplement 1. Identification of aIPg neurons in the hemibrain dataset. (A - B) Skeleton renderings of 26 putative aIP-g neurons described in Cachero et al. (2010) identified in the hemibrain dataset. The 11 aIPg type $1-3$ neurons are displayed in green with rest of the putative aIPg neurons shown in grey. Note that the aIP-g cluster described in Cachero et al. (2010) included neurons with cell bodies in the anterior of the brain, none of which were traced for this project in the FAFB dataset or included in this grouping the hemibrain as the neurons in our split-GAL4 lines only contained posterior located cell bodies. 


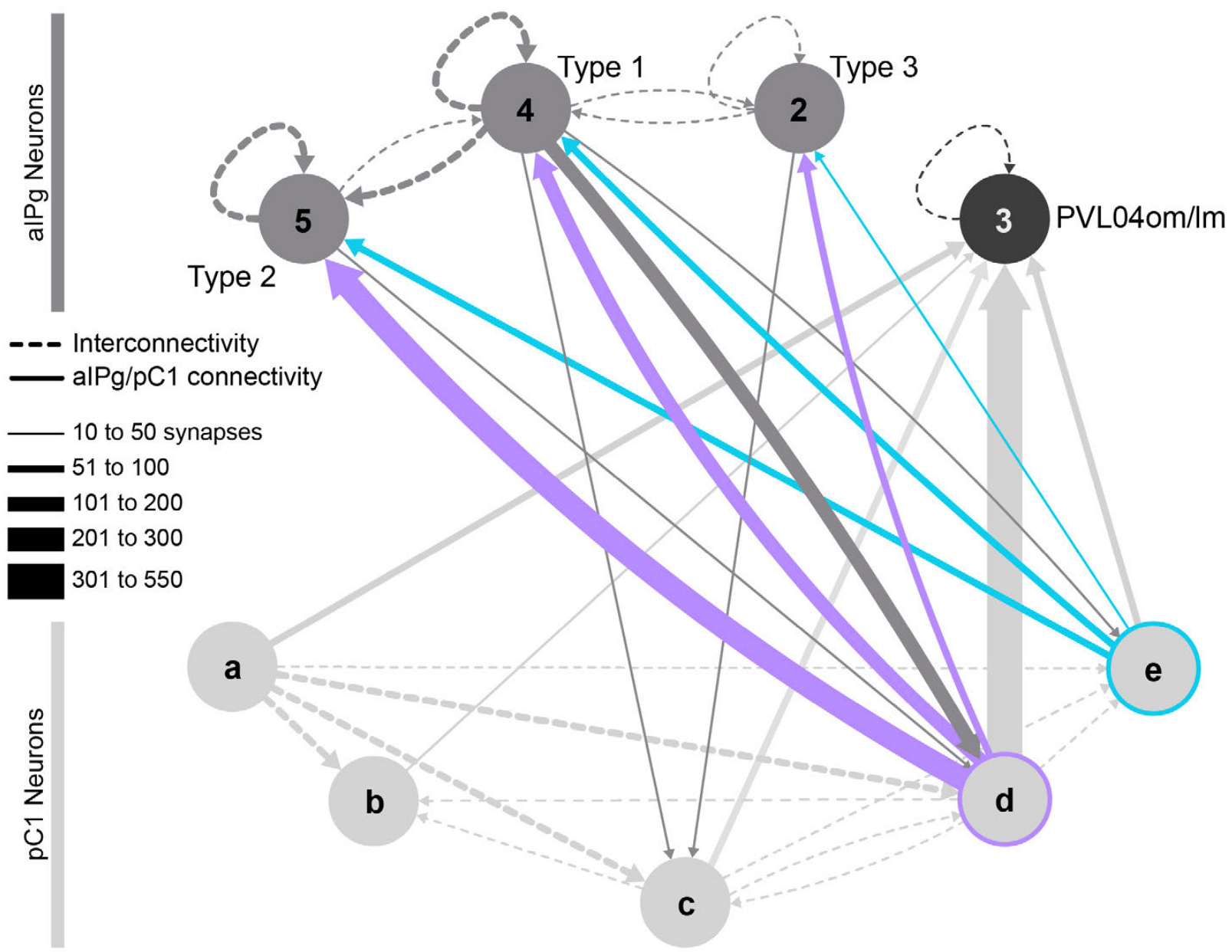

Figure 8 - figure supplement 1. Connections between pC1a-c neurons and pC1d and pC1e.

$\mathrm{pCla}, \mathrm{pClb}$ and $\mathrm{pClc}$ neurons have been added to the diagram shown in Figure 8. pCla and $\mathrm{pClc}$ provide weak input to $\mathrm{pC} 1 \mathrm{~d}$, and all $\mathrm{pC} 1$ neurons provide input to the three PVL04om/lm neurons, which are among $\mathrm{pCld}$ 's strongest downstream targets. Connections were thresholded at 10 synapses. 
bioRxiv preprint doi: https://doi.org/10.1101/2020.05.27.118810; this version posted May 27, 2020. The copyright holder for this preprint (which was not certified by peer review) is the author/funder, who has granted bioRxiv a license to display the preprint in perpetuity. It is made available under aCC-BY 4.0 International license.

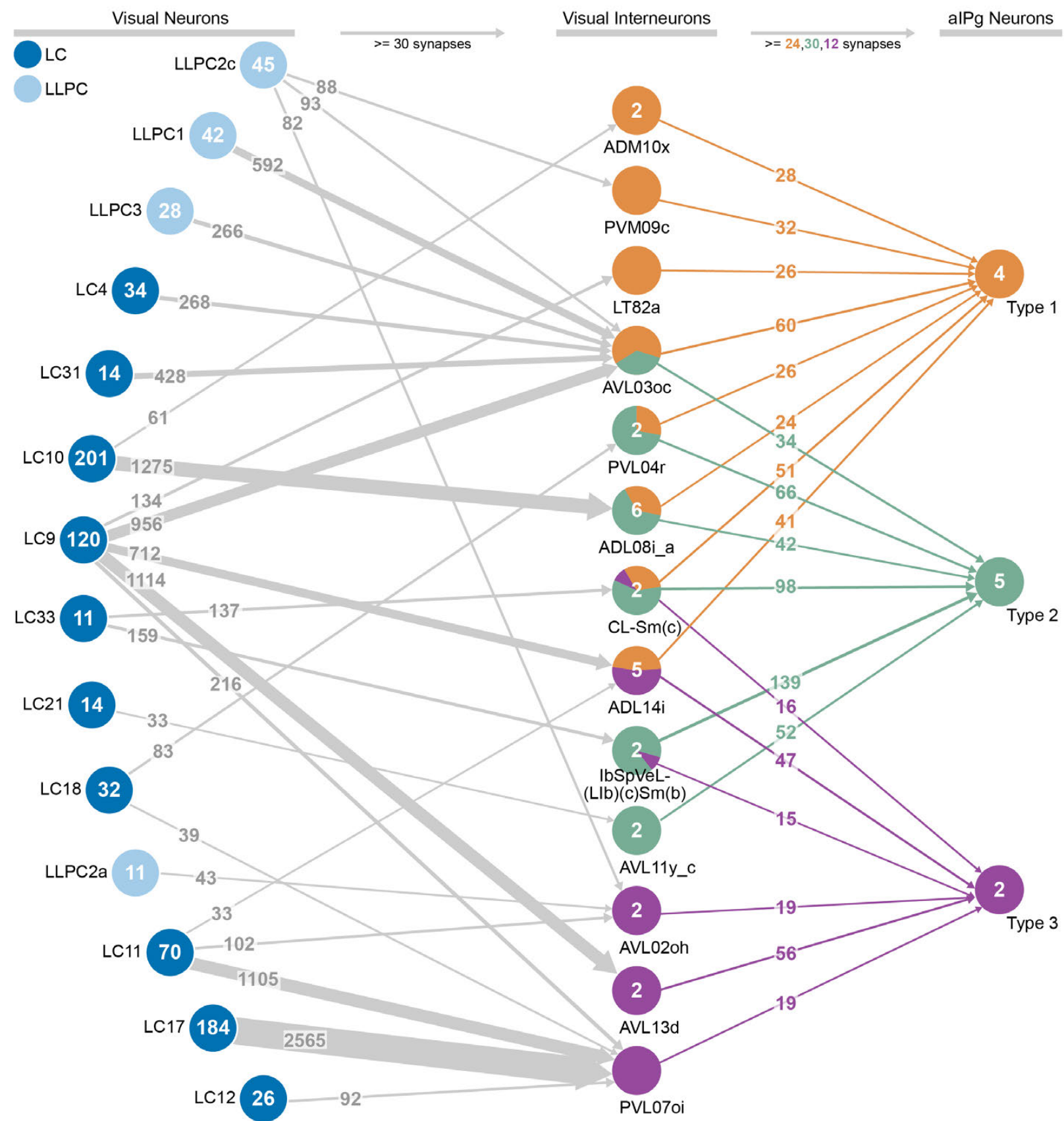

Figure 11 - figure supplement 1. Connections between visual projection neurons, interneurons, and aIPg type 1, type 2 and type 3 . Connections were thresholded at 30 combined synapses for each population of visual projection neurons to interneurons. Inputs to the aIPg neurons were thresholded at 6 synapses per cell, resulting in collective thresholds of 24,30 , and 12 synapses to type 1 , type 2 , and type 3 , respectively. 
964 Supplementary Table 1. Framewise performance of automated classifiers.

965

966 Supplementary Table 2. List of the top pre-synaptic inputs and post-synaptic outputs of the

96711 aIPg neurons identified. Thresholded at 57 synapses for the inputs and 150 synapses for the

968 outputs.

969

970 Supplementary Table 3. List of the top pre-synaptic inputs and post-synaptic outputs of

971 pC1d. Thresholded at 90 synapses for the inputs and 95 synapses for the outputs.

972

973 Supplementary File 4. Genotypes of the split-GAL4 lines used and cell counts for each of

974 the aIPg split-GAL4 lines.

975

976 Supplementary File 5. Sample size and statistics for behavioral analysis.

977

978 Video 1. Movie of control (EmptySS > Chrimson) flies with $0.1 \mathrm{~mW} / \mathrm{mm}^{2}$ light density

979 stimulation.

980

981 Video 2. Movie of stimulating flies from line labelling aIPg1-3 (aIPgSS1 > Chrimson) with

982

$0.1 \mathrm{~mW} / \mathrm{mm}^{2}$ light density.

983

984 Video 3. High speed video of two aIPgSS1>Chrimson female flies.

985

986 Video 4. High speed video of an aIPgSS1>Chrimson female fly with a Wt (Canton-S) male. 
988 Video 5. Movie of stimulating flies from line labelling pC1d (pC1dSS1 > Chrimson) with

$989 \quad 0.1 \mathbf{~ m W} / \mathbf{m m}^{2}$ light density.

990

991 Video 6. High speed video of two pC1dSS1>Chrimson female flies.

992

993 Video 7. Movie of the location of pre- and post-synaptic connections with aIPg1-3 neurons

994 based on the EM dataset. Skeleton renderings and synapse locations shown were taken from

995 NeuPrint and NeuTu.

996

997 Video 8. Movie of aIPg types 1 - 3 and their interconnectivity based on the EM dataset. The

998 number of neurons within the cell types depicted are in the circles and synapse number for each

999 connection is displayed next to the arrow in the cartoon.

1000

1001 Video 9. Movie of aIPg types 1 - 3 connectivity with pC1d and pC1e based on the EM

1002 dataset.

1003

1004 Video 10. Movie of the top pre-synaptic inputs to aIPg types $1-3$ based on the EM dataset.

1005

1006 Video 11. Movie of the top post-synaptic outputs of aIPg types $1-3$ based on the EM

1007 dataset.

1008

1009 Video 12. Movie of the top pre-synaptic inputs to pC1d based on the EM dataset. 
bioRxiv preprint doi: https://doi.org/10.1101/2020.05.27.118810; this version posted May 27, 2020. The copyright holder for this preprint (which

was not certified by peer review) is the author/funder, who has granted bioRxiv a license to display the preprint in perpetuity. It is made available under aCC-BY 4.0 International license.

1010

1011 Video 13. Movie of the top post-synaptic outputs of pC1d based on the EM dataset. 